\title{
A Search for the Higgs Boson in the ZH Dilepton Decay Channel at CDF II
}

\author{
by \\ Ravi Shekhar \\ Department of Physics \\ Duke University
}

Date:

\begin{tabular}{c} 
Approved: \\
\hline Assoc. Professor Ashutosh V. Kotwal, Advisor \\
\hline Professor Richard G. Palmer \\
\hline Professor Alfred T. Goshaw
\end{tabular}

Thesis submitted in partial fulfillment of the requirements for the degree of Master of Science in the Department of Physics in the Graduate School of Duke University 2009 
Copyright (c) 2009 by Ravi Shekhar

All rights reserved except the rights granted by the http://creativecommons .org/licenses/by/3.0/us/

(8.) Creative Commons Attribution 3.0 License 


\section{Abstract}

This dissertation describes a search for the Standard Model Higgs boson produced in association with the $Z$ boson via Higgs-strahlung at the CDF II detector at the Tevatron. At a Higgs boson mass between $100 \mathrm{GeV} / \mathrm{c}^{2}$ and $135 \mathrm{GeV} / \mathrm{c}^{2}$, the primary Higgs decay mode is to a pair of $b$ quarks. The associated $Z$ boson can decay to a pair of electrons or muons, allowing detection of a final event signature of two visible leptons and two $b$ quarks. This final state allows reduction of large QCD backgrounds compared to a hadronic $Z$ boson decay, leading to a more sensitive search. To increase sensitivity, standard model matrix element probabilities for $Z H$ signal and the dominant backgrounds are used as components to a likelihood fit in signal fraction.

In $2.7 \mathrm{fb}^{-1}$ of CDF II data, we see no evidence of production of a Higgs boson with a mass between $100 \mathrm{GeVc} c^{2}$ and $150 \mathrm{GeV} / \mathrm{c}^{2}$. Using the Feldman-Cousins technique to set a limit, at $95 \%$ coverage and a Higgs boson mass of $115 \mathrm{GeV} / \mathrm{c}^{2}$, the median expected limit was $12.1 \times \sigma_{S M}$ and a limit of $8.2 \times \sigma_{S M}$ was observed, where $\sigma_{S M}$ is the NNLO theoretical cross section of $p \bar{p} \rightarrow Z H \rightarrow l^{+} l^{-} b \bar{b}$ at $\sqrt{s}=1.96 \mathrm{TeV}$. Cross section limits are computed at a range of Higgs boson mass values between $100 \mathrm{GeV} / \mathrm{c}^{2}$ and $150 \mathrm{GeV} / \mathrm{c}^{2}$. 


\section{Contents}

$\begin{array}{lr}\text { Abstract } & \text { iii } \\ \text { List of Tables } & \text { vii } \\ \text { List of Figures } & \text { viii } \\ \text { Acknowledgements } & \text { xi } \\ 1 \quad \text { Introduction } & 1 \\ 2 \quad \text { Motivation and Theory } & 4\end{array}$

$2.1 \quad$ Standard Model . . . . . . . . . . . . . . . . . 5

2.1.1 Fundamental Particles $\ldots \ldots \ldots \ldots \ldots \ldots \ldots \ldots$

2.1.2 Standard Model Symmetries $\ldots \ldots \ldots \ldots \ldots \ldots \ldots$

2.2 Quantum Chromodynamics $\ldots \ldots \ldots \ldots \ldots \ldots \ldots$

2.3 Electroweak Interactions . . . . . . . . . . . . . . . 11

2.3.1 Goldstone Model. . . . . . . . . . . . . . . . . . . . . 12

2.3.2 Higgs Mechanism . . . . . . . . . . . . . . . . . 14

2.4 Higgs Phenomenology . . . . . . . . . . . . . . . . 16

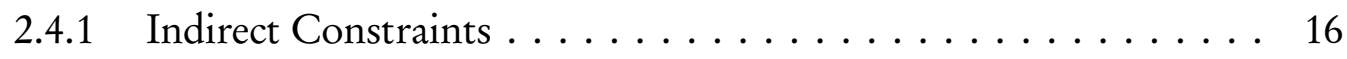

2.4.2 Tevatron Higgs Production $\ldots \ldots \ldots \ldots \ldots \ldots \ldots$

3 Experimental Apparatus $\quad 23$

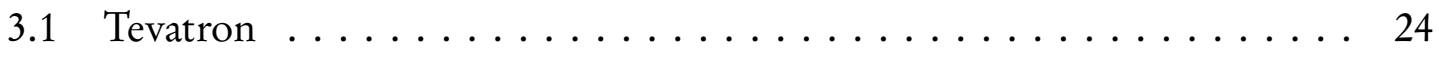

3.2 CDF II Detector $\ldots \ldots \ldots \ldots \ldots \ldots \ldots \ldots \ldots \ldots \ldots \ldots \ldots \ldots \ldots \ldots$ 
3.2.1 Cerenkov Luminosity Counter $\ldots \ldots \ldots \ldots \ldots \ldots \ldots$

3.2.2 Silicon Tracking . . . . . . . . . . . . . . . . . . . . . . 29

3.2.3 Central Outer Tracker . . . . . . . . . . . . . . . 30

3.2.4 Calorimeters ..................... 31

3.2 .5 Muon Detectors $\ldots \ldots \ldots \ldots \ldots \ldots \ldots \ldots \ldots$

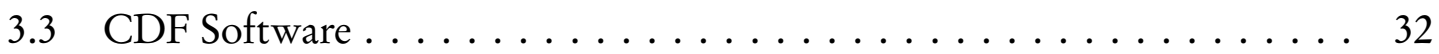

3.3.1 Event Triggers $\ldots \ldots \ldots \ldots \ldots \ldots \ldots \ldots \ldots \ldots \ldots \ldots$

3.3.2 Lepton Identification $\ldots \ldots \ldots \ldots \ldots \ldots \ldots \ldots$

3.3.3 Jet Modeling and Reconstruction . . . . . . . . . . . 35

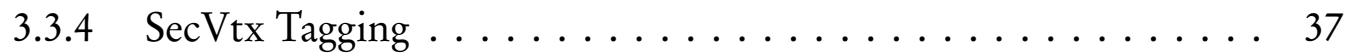

4 Event Selection $\quad 38$

4.1 Event Criteria . . . . . . . . . . . . . . . . . . . . . . . 39

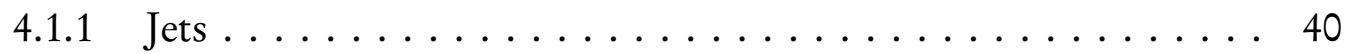

$4.1 .2 \quad b$-Tagging $\ldots \ldots \ldots \ldots \ldots \ldots \ldots \ldots \ldots \ldots \ldots \ldots \ldots \ldots \ldots$

4.2 Backgrounds ....................... 43

4.3 Signal Acceptance $\ldots \ldots \ldots \ldots \ldots \ldots \ldots \ldots \ldots$. . . . . . . . . 44

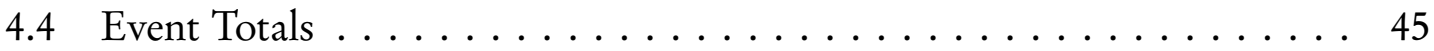

$4.4 .1 \quad \operatorname{Pretag} \ldots \ldots \ldots \ldots \ldots \ldots \ldots \ldots \ldots \ldots \ldots \ldots$

4.4.2 Double Loose $b$-Tag . . . . . . . . . . . . . . . . . 46

$4.4 .3 \quad$ Single Tight $b$-tag $\ldots \ldots \ldots \ldots \ldots \ldots \ldots \ldots \ldots$

5 Matrix Elements $\quad 49$

5.1 Matrix Element Approximations $\ldots \ldots \ldots \ldots \ldots \ldots \ldots$

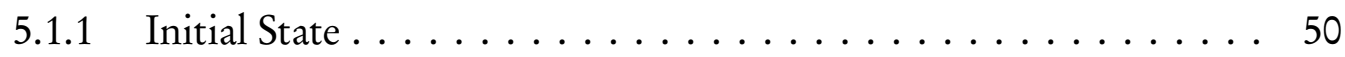

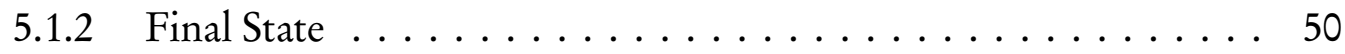

5.2 Jet Transfer Function $\ldots \ldots \ldots \ldots \ldots \ldots \ldots \ldots \ldots \ldots \ldots$ 


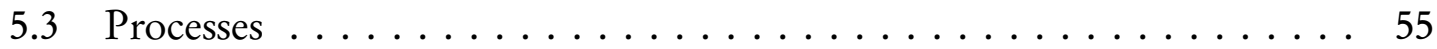

5.3.1 $Z H \rightarrow l^{+} l^{-} b \bar{b}$ and $Z Z \ldots \ldots \ldots \ldots \ldots \ldots \ldots$

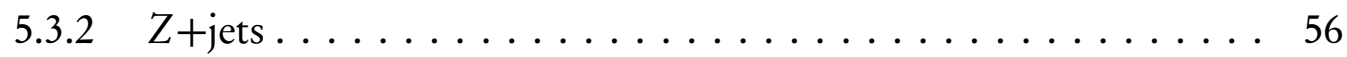

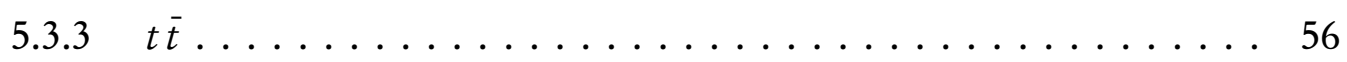

6 Analysis Technique $\quad 58$

6.1 Likelihood Fitting $-1 \ldots \ldots \ldots \ldots \ldots \ldots \ldots \ldots$

6.2 Resampling. . . . . . . . . . . . . . . . . . 59

6.3 Normalization . . . . . . . . . . . . . . . 60

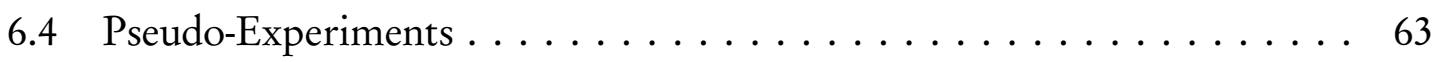

6.5 Likelihood Fitting $-2 \ldots \ldots \ldots \ldots \ldots \ldots \ldots \ldots$

6.5.1 Combined Tag Channels . . . . . . . . . . . . . 68

6.5.2 Separated Tag Channels . . . . . . . . . . . . . . . . 69

6.6 Feldman-Cousins Method . . . . . . . . . . . . . . . . . . 70

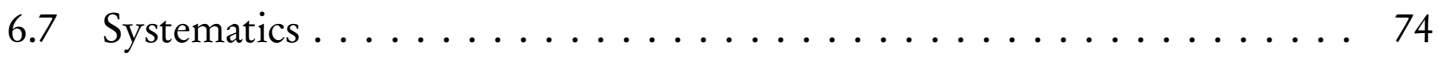

$\begin{array}{lll}7 & \text { Results } & 77\end{array}$

8 Conclusions \& Future Work $\quad 81$

A Additional Plots $\quad 83$

$\begin{array}{lr}\text { Bibliography } & 95\end{array}$ 


\section{List of Tables}

2.1 Fundamental Forces in the Universe $\ldots \ldots \ldots \ldots \ldots \ldots \ldots$

4.1 Summary of Event Criteria $\ldots \ldots \ldots \ldots \ldots \ldots \ldots \ldots$

4.2 Central electron trigger path . . . . . . . . . . . . . . . . . 39

4.3 Central muon trigger paths $\ldots \ldots \ldots \ldots \ldots \ldots \ldots \ldots$

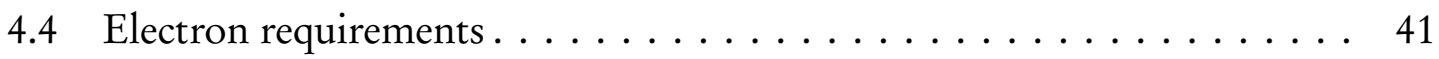

4.5 Muon requirements $\ldots \ldots \ldots \ldots \ldots \ldots \ldots \ldots \ldots \ldots \ldots$

4.6 Expected $Z H \rightarrow l^{+} l^{-} b \bar{b}$ events $\ldots \ldots \ldots \ldots \ldots \ldots \ldots \ldots$

4.7 Pretag background expectation $\ldots \ldots \ldots \ldots \ldots \ldots \ldots \ldots \ldots$

4.8 Double $b$-tag background expectation $\ldots \ldots \ldots \ldots \ldots \ldots \ldots$

4.9 Single $b$-tag background expectation $\ldots \ldots \ldots \ldots \ldots \ldots \ldots \ldots 47$

5.1 Transfer function constants $\ldots \ldots \ldots \ldots \ldots \ldots \ldots \ldots \ldots$

6.1 Normalization constants for matrix elements $\ldots \ldots \ldots \ldots \ldots . \ldots 3$

6.2 Expected Signal $/ \sqrt{\text { Background }}$ Table $\ldots \ldots \ldots \ldots \ldots \ldots \ldots 64$

6.3 Background $b$ tagging rates $\ldots \ldots \ldots \ldots \ldots \ldots \ldots \ldots \ldots$

6.4 Signal $b$ tagging rates $\ldots \ldots \ldots \ldots \ldots \ldots \ldots \ldots \ldots \ldots \ldots$

6.5 Rate Systematic scale factor . . . . . . . . . . . . . . 66

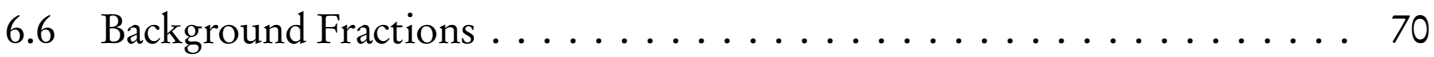

6.7 Rate Uncertainties . . . . . . . . . . . . . . . . . 75

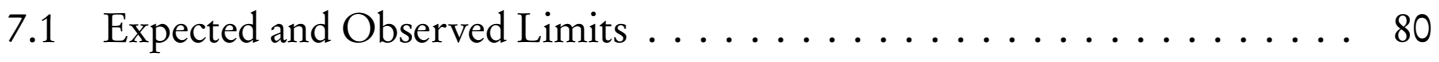




\section{List of Figures}

2.1 Fundamental particles in the Standard Model $\ldots \ldots \ldots \ldots \ldots \ldots$

2.2 Tree Level Standard Model Interactions $\ldots \ldots \ldots \ldots \ldots \ldots$

2.3 Vertices of QCD $\ldots \ldots \ldots \ldots \ldots \ldots \ldots \ldots \ldots \ldots \ldots \ldots \ldots \ldots \ldots \ldots \ldots \ldots$

2.4 A schematic diagram of the colors in QCD SU(3) group . . . . . . . 10

2.5 Meson color configuration . . . . . . . . . . . . . . . . 11

2.6 Baryon color configuration $\ldots \ldots \ldots \ldots \ldots \ldots \ldots \ldots \ldots \ldots$

$2.7 \quad V_{s}$ and $V_{s b}$ potentials $\ldots \ldots \ldots \ldots \ldots \ldots \ldots \ldots \ldots \ldots \ldots \ldots \ldots \ldots$

2.8 Goldstone Symmetry Breaking Potential . . . . . . . . . . . . . . 13

2.9 Theoretical Constraints on Higgs Mass $\ldots \ldots \ldots \ldots \ldots \ldots \ldots$

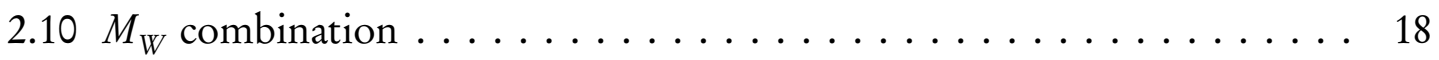

$2.11 m_{t o p}$ combination $\ldots \ldots \ldots \ldots \ldots \ldots \ldots \ldots \ldots \ldots \ldots \ldots \ldots \ldots \ldots \ldots \ldots$

2.12 Summary of Electroweak Constraints on the Higgs $\ldots \ldots \ldots \ldots$

2.13 Global Fit to electroweak data . . . . . . . . . . . . . . . 19

$2.14 p \bar{p} \rightarrow Z H$ Production Cross Section $\ldots \ldots \ldots \ldots \ldots \ldots \ldots$

2.15 Higgs Production Processes $\ldots \ldots \ldots \ldots \ldots \ldots \ldots \ldots \ldots \ldots$

2.16 Higgs Boson Branching Ratios . . . . . . . . . . . . . . 21

$2.17 \mathrm{ZH} \rightarrow l^{+} l^{-} b \bar{b}$ production mechanism $\ldots \ldots \ldots \ldots \ldots \ldots 22$

3.1 Tevatron Complex . . . . . . . . . . . . . . . . 24

3.2 Integrated luminosity at $\mathrm{CDF}$ II $\ldots \ldots \ldots \ldots \ldots \ldots \ldots \ldots$ 
$3.3 \mathrm{CDF}$ detector schematic $\ldots \ldots \ldots \ldots \ldots \ldots \ldots \ldots \ldots \ldots$

3.4 CDF Tracking Volume . . . . . . . . . . . . . . . . . . . . . . . . 29

3.5 Silicon detector barrel structure $\ldots \ldots \ldots \ldots \ldots \ldots \ldots \ldots$

3.6 Muon detector coverage $\ldots \ldots \ldots \ldots \ldots \ldots \ldots \ldots \ldots \ldots$

3.7 Jet energy scale $\ldots \ldots \ldots \ldots \ldots \ldots \ldots \ldots \ldots \ldots \ldots \ldots \ldots$

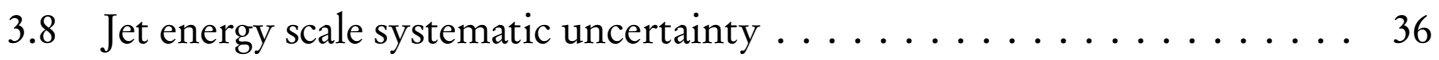

$4.1 \quad Z H$ Higgs decay $b$-quark kinematics $\ldots \ldots \ldots \ldots \ldots \ldots \ldots \ldots 42$

$4.2 t \bar{t}$ and Drell-Yan + jets Background Processes $\ldots \ldots \ldots \ldots \ldots \ldots 43$

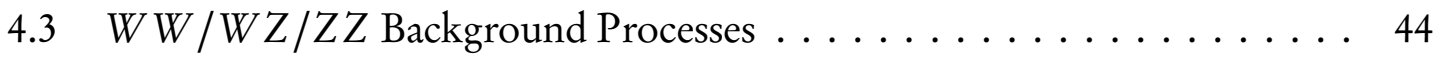

$4.4 \mathrm{ZH} \rightarrow l^{+} l^{-} b \bar{b}$ production mechanism $\ldots \ldots \ldots \ldots \ldots \ldots . \ldots 4$

4.5 Pretag channel kinematics $\ldots \ldots \ldots \ldots \ldots \ldots \ldots \ldots \ldots$

4.6 Double $b$-tag channel kinematics $\ldots \ldots \ldots \ldots \ldots \ldots \ldots \ldots . \ldots 7$

$4.7 \quad$ Single $b$-tag channel kinematics $\ldots \ldots \ldots \ldots \ldots \ldots \ldots \ldots .48$

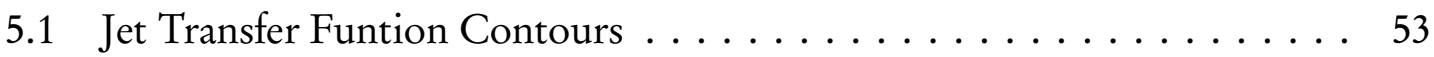

5.2 Parton and Jet Spectra . . . . . . . . . . . . . . . . . . . . 54

6.1 A single log-likelihood curve $\ldots \ldots \ldots \ldots \ldots \ldots \ldots \ldots \ldots$

6.2 Normalization histogram in $S_{m} \ldots \ldots \ldots \ldots \ldots \ldots \ldots \ldots \ldots \ldots \ldots$

6.3 Pseudo-experiment Likelihood $\ldots \ldots \ldots \ldots \ldots \ldots \ldots \ldots \ldots$

6.4 Pseudo-experiment Log-likelihood $\ldots \ldots \ldots \ldots \ldots \ldots \ldots \ldots$

6.5 Measured Signal Fraction Peak Histograms … . . . . . . . . . 72

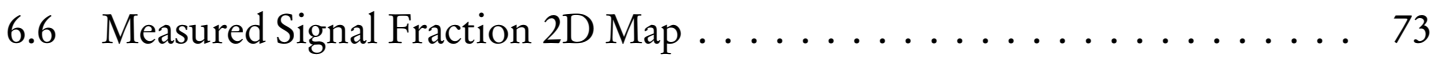

6.7 Feldman-Cousins Ratio . . . . . . . . . . . . . . . . . . . . 74

6.8 Shape Systematic Effects . . . . . . . . . . . . . . . . . . 76

7.1 Data Likelihood Curve . . . . . . . . . . . . . . . . . . . . 77

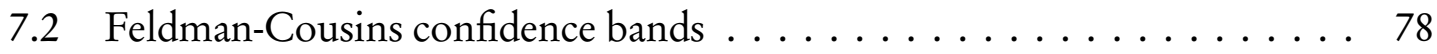


7.3 Expected distribution of limits $\ldots \ldots \ldots \ldots \ldots \ldots \ldots \ldots$

$7.4 p \bar{p} \rightarrow Z H \rightarrow l^{+} l^{-} b \bar{b}$ limits at $95 \%$ coverage. $\ldots \ldots \ldots \ldots \ldots$

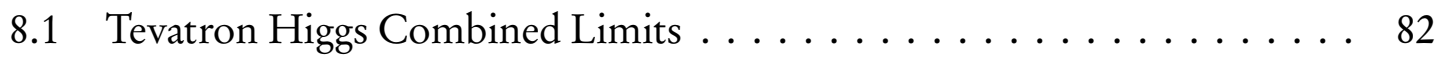

A.1 $M_{H}=100 \mathrm{GeV} / \mathrm{c}^{2} \ldots \ldots \ldots \ldots \ldots \ldots \ldots \ldots \ldots \ldots \ldots$

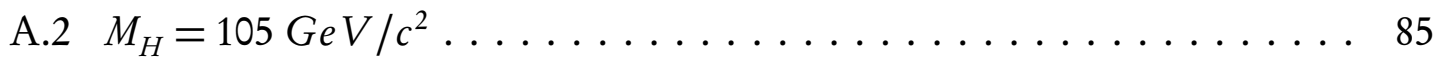

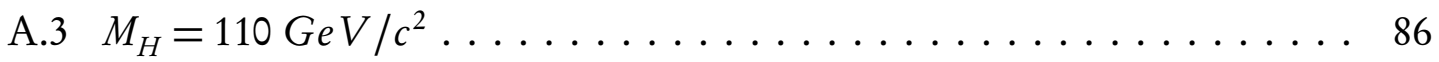

A.4 $M_{H}=115 \mathrm{GeV} / \mathrm{c}^{2} \ldots \ldots \ldots \ldots \ldots \ldots \ldots \ldots \ldots \ldots \ldots$

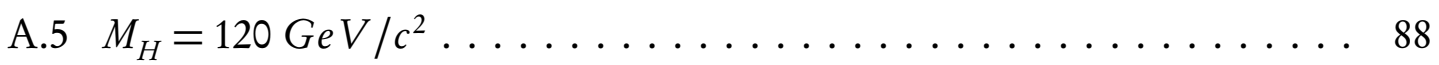

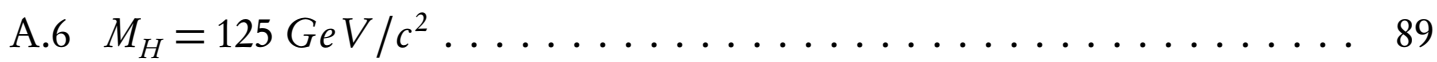

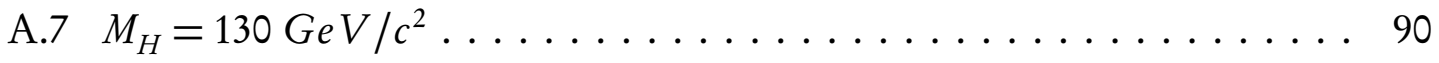

A.8 $\quad M_{H}=135 \mathrm{GeV} / \mathrm{c}^{2} \ldots \ldots \ldots \ldots \ldots \ldots \ldots \ldots \ldots \ldots \ldots \ldots \ldots \ldots \ldots \ldots \ldots$

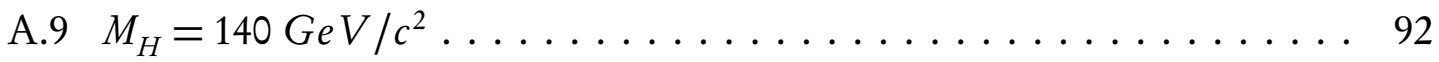

A.10 $M_{H}=145 \mathrm{GeV} / \mathrm{c}^{2} \ldots \ldots \ldots \ldots \ldots \ldots \ldots \ldots \ldots \ldots \ldots \ldots \ldots \ldots \ldots$

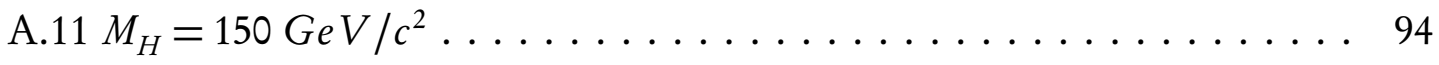




\section{Acknowledgements}

A great many people have helped make this work possible and I cannot possibly thank them all.

My advisor Ashutosh Kotwal, who was very patient, always willing to explain when I did not understand, and stuck up for me. His detail oriented approach really made me appreciate the big picture a great deal. Bo Jayatilaka, who guided the $\mathrm{ZH}$ analysis from start to completion with me, answering innumerable questions along the way. Daniel Whiteson, who handed me a big part of the analysis with a bow on top, and was always available for questions. Ben Kilminster and Shalhout Shalhout, who provided insight and comparison at our weekly meetings. My dissertation committee, Al Goshaw, and Richard Palmer for their time and guidance on short notice.

Yu Zeng and Seth Henshaw for those invaluable late night homework sessions. My undergraduate P122 professor, Mano Singham, for dazzling me with the derivation of light propagation from Maxwell's Equations, and unknowingly inspiring me to study physics. My undergraduate advisor, Corbin Covault, who gave me a feel for research in

the first place. My family for their support these past eight years despite the distance. My Naniji for her incredible strength and resolve through difficult times. Elizabeth for her support and friendship these past two years, and Maria, wherever she may be. 


\section{1}

\section{Introduction}

Over a century ago, future Nobel Laureate Albert A. Michelson wrote the famous words $[1]$

The more important fundamental laws and facts of physical science have all been discovered, and these are now so firmly established that the possibility of their ever being supplanted is exceedingly remote $[\ldots]$ our future discoveries must be looked for in the sixth place of decimals.

At the end of the 19th century, physics seemed like a complete, self-consistent theory. All of known physics could be predicted by the classical theories of mechanics, fields, electromagnetism and thermodynamics. Nature was predicted so well that it led many, including Michelson and Lord Kelvin to believe there was nothing more to discover.

Yet two problems lingered. The xther could not be detected in an experimental setting, despite ever increasing sensitivity of measurements. And blackbody radiation of resonant cavities could not be explained without an ultraviolet divergence. The solution to the two problems came from Special Relativity and Quantum Mechanics respectively. In the 1940s these two ideas were integrated into Quantum Electrodynamics, an extremely 
accurate, predictive theory of the interaction of light and matter. Shortly thereafter, large numbers of new particles were observed at particle accelerators leading to the coining of the term the particle zoo. There were many competing theories to explain the particle zoo, and eventually one was selected by a combination of experiment and theoretical progress. That theory is now called the standard model of particle physics (SM).

The SM was developed with knowledge of a significant part of the particle zoo, so it fit the known particles quite well. It predicted several new particles which had not yet been observed, including what came to be known as the $W$ and $Z$ bosons, the gluon, and the bottom and top quarks. Each of these particles has been experimentally observed between the mid 1970s and 2000, making the SM one of the most successful and longest lasting theories of Modern Physics.

But the SM had one flaw; it required all fundamental particles to be massless. Because we know this to be empirically false, there must be a mechanism through which otherwise massless fundamental particles acquire mass. In 1964, Peter Higgs, along with five others proposed [2] that there is a field (now known as the Higgs Field) that permeates the universe and has a nonzero vacuum expectation value (vev). This nonzero vev imparts mass to all particles with electroweak charge. The particles behave as if they are swimming through the thick, viscous Higgs field. This viscous field coupling produces terms in equations which are indistinguishable from inertial mass. This idea came to be known as the Higgs Mechanism and was incorporated into the SM, albeit with the drawback that each fundamental particle's coupling to the Higgs field is a free parameter in the SM. More on the Higgs and how it relates to the SM is presented in chapter 2.

It is interesting to consider the parallels between now and a century ago. Currently, the SM seems predictive of all of particle physics (with the possible exception of massive neutrino, see [3]). The only unobserved particle from the minimal, self-consistent SM is the Higgs boson. In both cases there seems to be a framework for understanding and predicting large parts of the physical world. Only a small piece of the puzzle is missing. 
Discovery of this piece would be another success to an amazingly successful theory. On the other hand, discovery of a null result would force a change in the SM and potentially open the door to significant new Physics. Regardless of the outcome, there is a gain in understanding of the universe. This makes the Higgs boson worth searching for.

This dissertation presents a search for the Higgs Boson produced in conjunction with the $Z$ boson via Higgs-strahlung at the Collider Detector located at the Tevatron at Fermi National Accelerator Laboratory (CDF at FNAL), located outside Chicago, Illinois. The salient points of the detector apparatus and software is presented in chapter 3. Radiative corrections to other parameters in the SM such as the $W$ boson mas and the top quark mass predict a Higgs boson with a mass between 50 and $200 \mathrm{GeV} / \mathrm{c}^{2}$. The $Z H$ associated production search presented in this dissertation has good sensitivity from 100 to 130 $\mathrm{GeV} / \mathrm{c}^{2}$, and is considered a 'low mass' Higgs search.

For Higgs boson masses between 100 and $135 \mathrm{GeV} / \mathrm{c}^{2}$, the Higgs primarily decays to a pair of $b$ quarks, $b \bar{b}$. The associated $Z$ boson decays to $e$ or $\mu$ approximately $7 \%$ of the time. The event signature to look for is a pair of $b$ quark jets and a pair of opposite sign $\left(e^{ \pm}, \mu^{ \pm}\right)$leptons. The dilepton requirement in this event signature allows reduction of large QCD backgrounds compared to $Z \rightarrow$ hadrons (See chapter 4). However, this requirement is not so tight as to allow a simple counting experiment to be used; advanced analysis techniques are still required to maximize sensitivity.

The advanced analysis technique used is a likelihood fitter with the signal and background matrix element probabilities for each event. Matrix elements, presented in chapter 5 , present a way of analyzing events somewhat independent from standard collaboration analysis tools, potentially allowing for semi-independent confirmation of results. The likelihood fitting technique used is presented in chapter 6. Finally, the results from this search are presented in chapter 7 . 


\section{2}

\section{Motivation and Theory}

We currently believe there are four fundamental forces in the universe, given in Table 2. The two nuclear forces have a characteristic scale The SM attempts to predict the strong,

\begin{tabular}{lccc}
\hline Force & Theory & Strength & Scale \\
\hline Strong Nuclear & Chromodynamics & $\sim 10$ & $10^{-15} \mathrm{~m}$ \\
Electromagnetic & Electrodynamics & $\sim 10^{-2}$ & $\infty$ \\
Weak Nuclear & Flavordynamics & $\sim 10^{-13}$ & $10^{-18} \mathrm{~m}$ \\
Gravity & Geometrodynamics & $\sim 10^{-42}$ & $\infty$ \\
\hline \multicolumn{2}{c}{ Table 2.1: Fundamental Forces in the Universe [4] }
\end{tabular}

weak and electromagnetic forces, as gravity is currently not entirely reconciled with Quantum Mechanics. One of the key findings is that above a certain energy $\left(\Lambda_{E W} \sim 100\right.$ GeV), the weak and electromagnetic forces unify into the electroweak force. Based on what are called Grand Unified Theories (GUTs), we believe at still higher energies $\left(\Lambda_{\mathrm{GUT}} \sim 10^{15} \mathrm{Ge} V\right)$, the electromagnetic, strong and weak nuclear forces will merge into a single force.

We believe the Higgs boson to be on the same scale as $\Lambda_{E W} \sim 100 \mathrm{Ge} V$, and therefore given the center of mass energy of $1.96 \mathrm{Te} V$ at the Tevatron, we can reasonably search 
for the Higgs.

\subsection{Standard Model}

The major theoretical underpinnings of the Standard Model (SM) have been largely unchanged for almost forty years (with the significant exception of nonzero neutrino mass, see [3]). By the late 1970s, the Lagrangian for the Standard Model had been written down in its current form. Figure 2.1 summarizes the fundamental particles along with their crucial properties like mass, charge, and spin. With the exception of the Higgs, hereafter abbreviated as $H$, all of the particles predicted have been discovered.

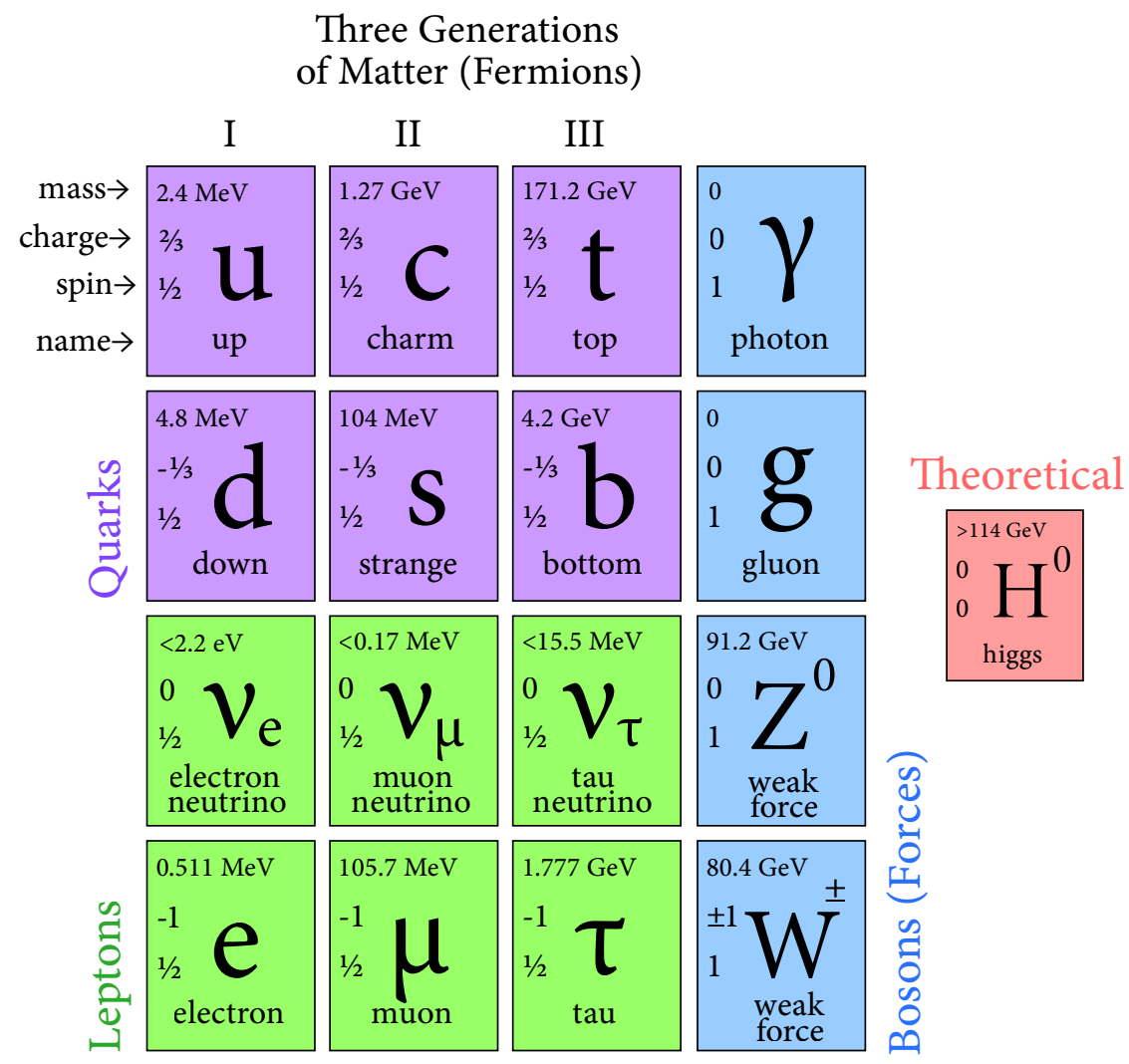

FIGURE 2.1: The minimum set of particles predicted by the Standard Model. Quarks and Leptons are fermions with spin $=1 / 2$ and the four bosons have spin 1. The Higgs Boson, which has not yet been observed has spin 0 . Figure from [5] 


\subsubsection{Fundamental Particles}

Particles can be classified according to the spin they have, either integer spin $(0,1,2, \ldots)$, or half-integer spin $\left(\frac{1}{2}, \frac{3}{2}, \ldots\right)$. Particles with integer spin are bosons and particles with half integer spin are fermions. Bosonic fields and fermionic fields transform differently under Lorentz Transforms, and have opposite symmetrization requirements for systems of identical particles. All particles that make up matter are fermions, whereas the particles that carry forces are bosons.

Looking at Figure 2.1, it is apparent that there is a left to right symmetry in the fermions. Each column is a generation, and each successive generation has particles with higher masses. The number of generations is not set by the SM, but all indications are that there are only three generations of matter. This symmetry between generations is called flavor symmetry and is broken by the fact that the particles have masses. Of course, in the high mass limit, flavor symmetry is regained between generations, which is often a useful fact when calculating cross sections and branching ratios.

\section{Fermions}

There are two classes of fermions, quarks and leptons. As seen in Figure 2.1, there are six quarks and six leptons, as well as corresponding antiparticles.

The six quarks are: up $(u)$, down $(d)$, charm (c), strange $(s)$, top $(t)$, and bottom (b), and their oppositely charged antiparticles form the hadronic sector of the SM. Quarks couple to all four force carrying bosons (because they carry both color and electroweak charge) and occur in isospin doublets for each generation. Interestingly, the quark eigenstates that interact with the $W$ and $Z$ are linear combinations of the mass eigenstates that interact with the gluon. The eigenstates are related by the CKM Matrix [6]. Because of the nature of the color charge gluon interaction, free quarks are not observed in nature, existing only in bound states of two (mesons) or three (baryons) quarks.

The six leptons are $\nu_{e}, \nu_{\mu}, \nu_{\tau}, e, \mu$, and $\tau$, and they come in isospin doublets like the 
quarks. The first three are neutral neutrinos and the others are charged leptons. Unlike quarks, leptons do not carry color charge, only electroweak charge. Consequently, they can exist as free particles. The neutrinos are believed to be left handed particles and therefore only couple to left handed $W$ and $Z$ bosons. The charged leptons couple to both left and right handed $W, Z$ and $\gamma$ bosons. More information on the SM predicted couplings is given in Figure 2.2.

\section{Bosons}

Bosons are the force carriers and have integer spin. The photon, $\gamma$ is the most well known and is the massless force carrier for the electromagnetic force. The $Z$ boson, a carrier of the weak force, has no electric charge and behaves much like a very massive $\gamma$, with a mass of $91.2 \mathrm{GeV} / \mathrm{c}^{2}$. Both are their own antiparticles, unlike the $W$, also a carrier of the weak force, which comes in two forms, $W^{+}$and $W^{-}$. Unlike the $\gamma, Z$ and the $W$ interact with each other, making the weak force non-abelian. Finally, the gluon is the carrier of the strong force, and is a massless bi-colored boson. Because it carries color charge, it can interact with other gluons, thus making the strong force non-abelian as well. The Higgs boson, still theoretical, is the quantum of the scalar Higgs field. It couples to itself, and to all massive particles. Couplings can be seen in Figure 2.2.

\subsubsection{Standard Model Symmetries}

The Standard Model is formulated as a gauge theory. Gauge theories are elegant because once the gauge fields have been postulated, the interactions between the fields are a property of the gauge symmetry group. The SM is a

$$
\mathrm{SU}(3)_{C} \times \mathrm{SU}(2)_{L} \times \mathrm{U}(1)_{Y}
$$

theory. The $\mathrm{SU}(3)$ has a subscript $C$ to indicate it represents the Quantum Chromodynamics (QCD) sector of the SM. This sector is also known as the colored sector because of the analogous relationship between the three colors of quarks and the three different 


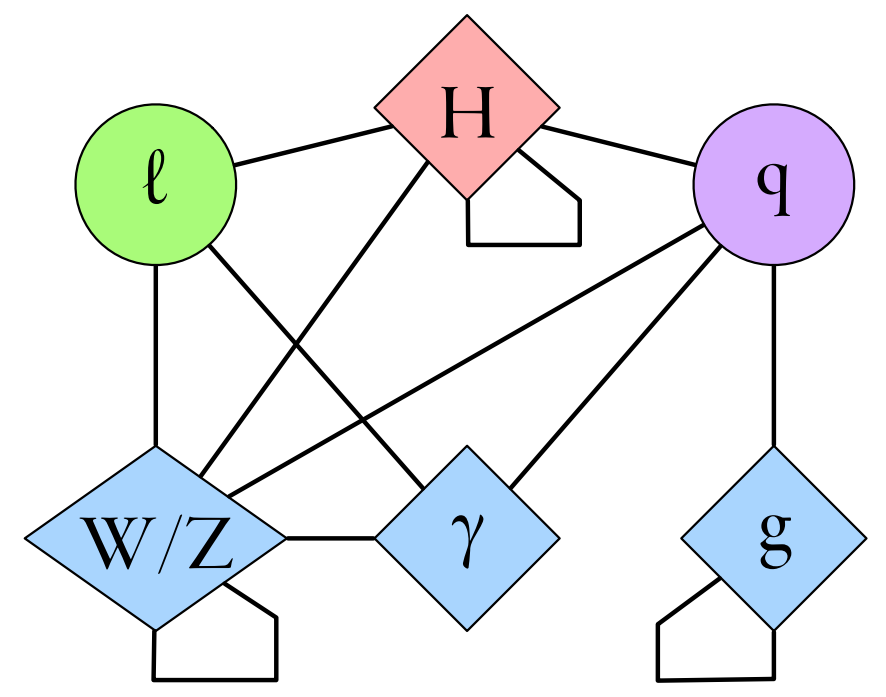

Figure 2.2: Tree level interactions in the Standard Model. Noticeably the $H, W$, and $g$ all have self-couplings, making the fields non-abelian.

additive components of white light: red, green and blue. The $\mathrm{SU}(2)$ group forms the weak sector and has the subscript $L$ to indicate that the group contains left-handed weak isospin doublets. The $\mathrm{U}(1)$ forms the electromagnetic sector and has the subscript $Y$ to indicate the group forms right-handed hypercharge singlets. The combined $\mathrm{SU}(2) \times \mathrm{U}(1)$ groups form the electroweak force.

From the gauge group, tree level interactions can be easily predicted. More detail for each sector will be given individually, but the tree level interactions between particles in the SM are given in Figure 2.2. The diagram can be summarized as

- The $W / Z$, and $g$ bosons have self coupling, indicating that the associated fields are non-abelian.

- Both quarks and leptons interact with the electroweak and Higgs sectors, coupling to $Z, W, \gamma$, and $H$ bosons.

- Gluons only couple to quarks and other gluons.

- The $W$ and $Z$ couple to the $H$, giving them mass, but the $\gamma$ and $g$ are massless. 


\subsection{Quantum Chromodynamics}

Quantum Chromodynamics is the study of the strong force between quarks and gluons. The vertices of QCD are given in Figure 2.3. Gluons are the massless force carrying bosons, and are bicolored, whereas quarks carry a single color charge. The coupling of particles via the strong force is given by [7]

$$
\alpha_{s}\left(q^{2}\right)=\frac{12 \pi}{\left(33-2 n_{f}\right) \log \left(q^{2} / \Lambda_{q c d}^{2}\right)}
$$

where $\Lambda_{q c d} \sim 200 \mathrm{MeV}$ and $n_{f}$ is the number of quark flavors.

The most unusual thing about the strong force is that as distance between the particles
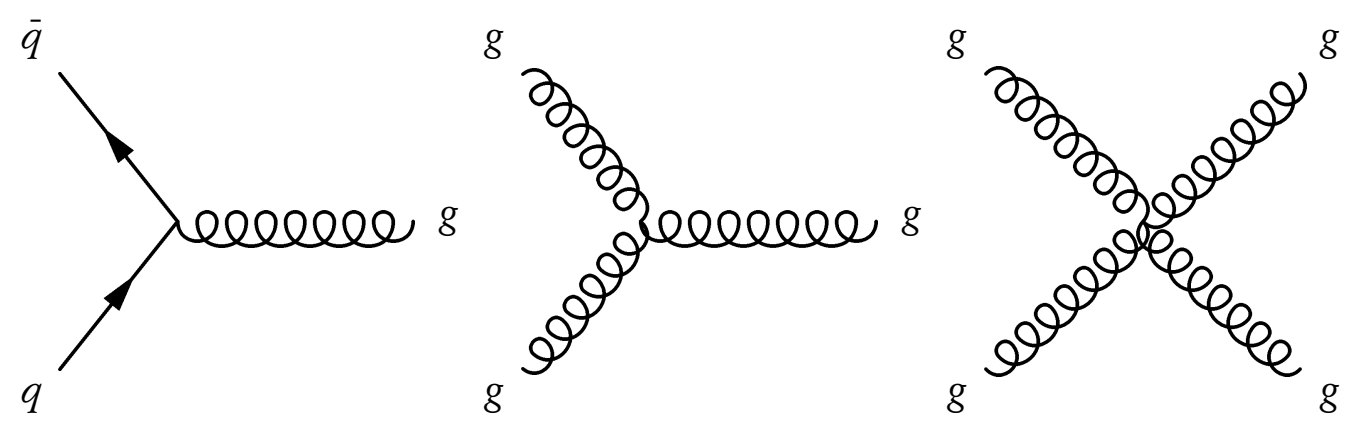

FIGURE 2.3: The vertices of QCD show quark-gluon and gluon-gluon coupling.

increases, the force between them also increases. This leads to the twin phenomena of confinement and asymptotic freedom. Confinement requires that free quarks do not exist; only quark bound states can exist. Asymptotic freedom states that in the limit of high energies (or alternatively small distances), quarks propagate as free particles, which is the logical extension of a force that increases with distance. This allows perturbation theory to be used for high center of mass energy scattering, like what is observed at the Tevatron. Figure 2.4 gives a rough schematic description of the QCD color states. Each of the three basis states can be added with complex coefficients, which is not shown on 


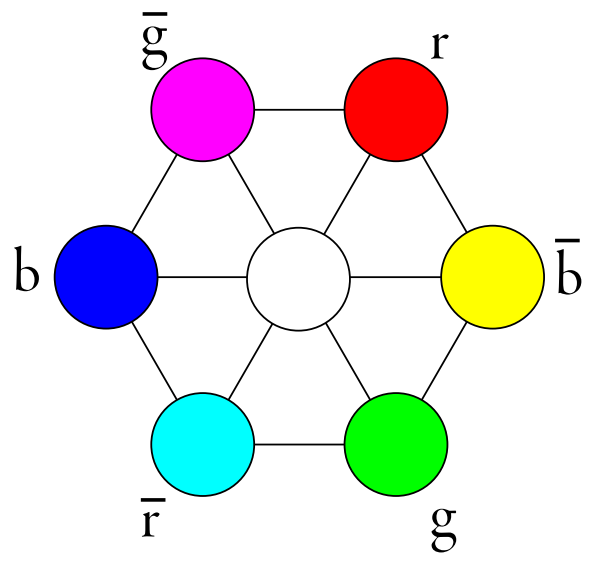

FIGURE 2.4: A schematic diagram of the colors in QCD SU(3) group

the diagram. However, from the figure, it is evident that there is more than one way to achieve a colorless (white) state.

Confinement requires colored gluons and colorless quark bound states. The three basis states can be combined into nine independent states. There are eight colored octet states (equation 2.3) and a colorless singlet state $(r \bar{r}+b \bar{b}+g \bar{g}) / \sqrt{3}$. The colorless state is not an allowed gluon state, so there are eight distinct allowed gluons. One convention defines the gluons as

$$
\begin{array}{ll}
(r \bar{b}+b \bar{r}) / \sqrt{2} & -i(r \bar{b}-b \bar{r}) / \sqrt{2} \\
(r \bar{g}+g \bar{r}) / \sqrt{2} & -i(r \bar{g}-g \bar{r}) / \sqrt{2} \\
(b \bar{g}+g \bar{b}) / \sqrt{2} & -i(b \bar{g}-g \bar{b}) / \sqrt{2} \\
(r \bar{r}+b \bar{b}) / \sqrt{2} & (r \bar{r}+b \bar{b}-2 g \bar{g}) / \sqrt{6}
\end{array}
$$

Correspondingly, there are two ways for quark bound states to achieve a colorless final state. The first is a meson (Figure 2.5), which is a combination of a color and the corresponding anti-color, such as $r \bar{r}$. The second is a baryon (Figure 2.6), which is an equal combination of the three colors (or anti-colors) like $r g b$. The QCD Lagrangian is 


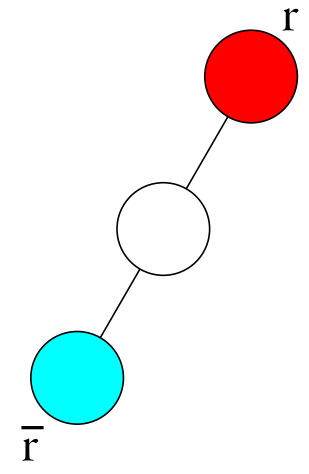

FIGURE 2.5: A possible meson color configuration

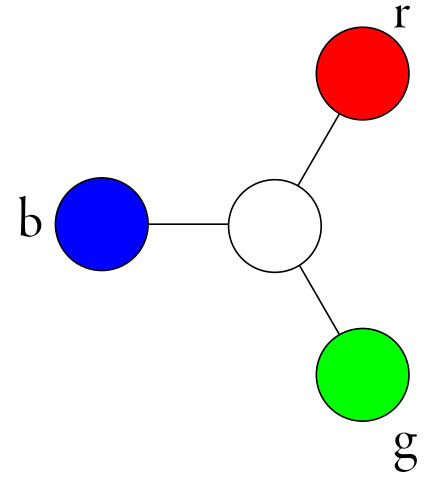

FIGURE 2.6: A possible baryon color configuration

given by [8]

$$
\mathscr{L}_{q c d}=-\frac{1}{4} F_{a}^{\mu \nu} F_{a \mu \nu}+\bar{\psi}_{j}\left(i \gamma_{\mu} D_{j k}^{\mu}-M_{j} \delta_{j k}\right) \psi_{k}
$$

where the covariant derivative is

$$
D_{j k}^{\mu}=\delta_{j k} \partial^{\mu}+i g\left(T_{a}\right)_{j k} G_{a}^{\mu}
$$

and $F_{a}^{\mu \nu}$ is the ath field strength tensor, $M$ is the quark mass matrix, $g$ is the strong coupling constant, and $T$ is the $\mathrm{SU}(3)$ generator matrices.

\subsection{Electroweak Interactions}

The electroweak interaction has the symmetry group $\mathrm{SU}(2)_{L} \times \mathrm{U}(1)_{Y}$. Weak Isospin $\left(T_{L}\right)$ and hypercharge $(Y)$ are the generators of the transformations. They relate to normal electromagnetic charge through the relation

$$
Q=T_{3}+\frac{1}{2} Y
$$

where $T_{3}$ is the third component of weak isospin. The electroweak Lagrangian is

$$
\mathscr{L}_{\text {ew }}=-\frac{1}{4} W^{\mu \nu} W_{\mu \nu}-B^{\mu \nu} B_{\mu \nu}+\bar{\psi} i \gamma^{\mu} D_{\mu} \psi
$$


with the covariant derivative

$$
D_{\mu}=\partial_{\mu}+i g W_{\mu} T+\frac{1}{2} i g^{\prime} B_{\mu} Y
$$

where $B_{\mu \nu}=\partial_{\mu} B_{\nu}-\partial_{\nu} B_{\mu}$, like the Maxwell field tensor. The $B$ field represents the gauge field corresponding to the $\mathrm{U}(1)_{Y}$. The $\mathrm{SU}(2)$ corresponding gauge field is $W_{\mu \nu}$, defined as

$$
W_{\mu \nu}=\partial_{\mu} W_{\nu}-\partial_{\nu} W_{\mu}-g W_{\mu} \times W_{\nu}
$$

The $W$ and $B$ fields mix (see section 2.3.2) through a mixing angle $\theta_{W}$ (also known as the Weinberg angle). The third component of weak isospin mixes with $B$ as

$$
\left(\begin{array}{l}
Z_{\mu} \\
A_{\mu}
\end{array}\right)=\left(\begin{array}{cc}
\cos \theta_{W} & -\sin \theta_{W} \\
\sin \theta_{W} & \cos \theta_{W}
\end{array}\right)\left(\begin{array}{l}
W_{\mu}^{3} \\
B_{\mu}
\end{array}\right)
$$

creating the familiar Maxwell field $A_{\mu}$ and the $Z$ boson field. Choosing $g^{\prime}=g \tan \theta_{W}$ and $e=g \sin \theta_{W}$, the observable $W$ is related to the ladder operators in $\mathrm{SU}(2)$, leading to a complete definition of all four fields as equations $2.11-2.13$

$$
\begin{aligned}
W_{\mu}^{ \pm} & =\frac{1}{\sqrt{2}}\left(W_{\mu}^{1} \mp i W_{\mu}^{2}\right) \\
Z_{\mu} & =\frac{-g^{\prime} B_{\mu}+g W_{\mu}^{3}}{\sqrt{g^{2}+g^{\prime 2}}} \\
A_{\mu} & =\frac{g B_{\mu}+g^{\prime} W_{\mu}^{3}}{\sqrt{g^{2}+g^{\prime 2}}}
\end{aligned}
$$

\subsubsection{Goldstone Model}

Before attacking the full complexities of the SM, it is useful to consider a simplified case of symmetry breaking from a nonzero vacuum expectation value. Following the treatment of Goldstone [9], let us posit the existence of a complex scalar field $\hat{\phi}$.

$$
\hat{\phi}=\frac{1}{\sqrt{2}}\left(\hat{\phi}_{1}-i \hat{\phi}_{2}\right) \quad \hat{\phi}^{\dagger}=\frac{1}{\sqrt{2}}\left(\hat{\phi}_{1}+i \hat{\phi}_{2}\right)
$$


with the Lagrangian density

$$
\hat{\mathscr{L}}_{G}=\left(\partial_{\mu} \hat{\phi}^{\dagger}\right)\left(\partial^{\mu} \hat{\phi}\right)-\hat{V}(\hat{\phi})
$$

Now we consider two cases, $\hat{V}_{s}(\hat{\phi}), \hat{V}_{s b}(\hat{\phi})$

$$
\hat{V}_{s}=\frac{1}{4} \lambda\left(\hat{\phi}^{\dagger} \hat{\phi}\right)^{2}+\mu^{2} \hat{\phi}^{\dagger} \hat{\phi} \quad \hat{V}_{s b}=\frac{1}{4} \lambda\left(\hat{\phi}^{\dagger} \hat{\phi}\right)^{2}-\mu^{2} \hat{\phi}^{\dagger} \hat{\phi}
$$

Clearly the Lagrangian is invariant under transforms in $\mathrm{U}(1), e^{-i \alpha}$. And as shown in Figure 2.7, the symmetric potential $V_{s}$ has a minimum at $(\hat{\phi} \hat{\phi})_{\min }=0$. In a perturbative situation, there will be a Taylor expansion in $|\hat{\phi}|$ about the minimum. Because the minimum is zero, the classical average value of the $\left(\hat{\phi}^{\dagger} \hat{\phi}\right)$ field is zero as well. The

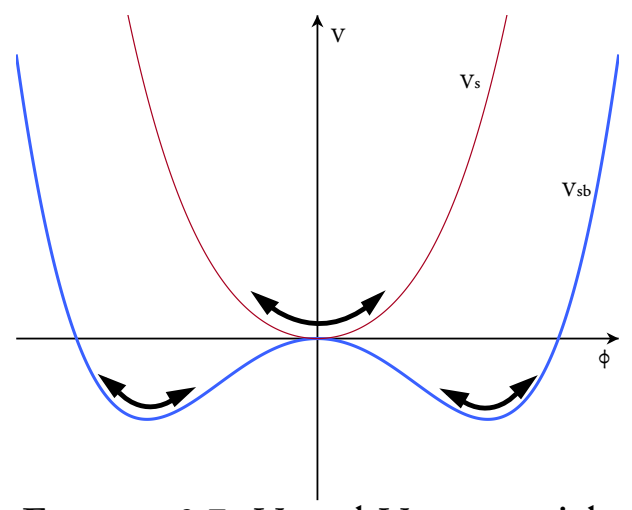

FIGURE 2.7: $V_{s}$ and $V_{s b}$ potentials

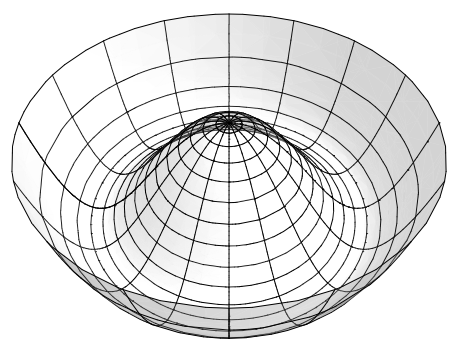

FIGURE 2.8: Goldstone Symmetry Breaking Potential

symmetry breaking potential $V_{s b}$ only changes the sign of the second term. This causes a qualitative change in behavior. We follow the same strategy as $V_{s}$, doing a taylor expansion about the minimum, $\left(\phi^{\dagger} \phi\right)_{\min }=2 \mu^{2} / \lambda$ which can be rearranged into $v=2|\mu| / \lambda^{1 / 2}$, where $v$ is the vacuum expectation value (vev). A plot of the symmetry breaking potential in the $\hat{\phi}_{1}, \hat{\phi}_{2}$ plane is Figure 2.8. Expanding about the minimum makes two modes immediately apparent, a $\theta$ mode with no restoring potential, and a radial mode with approximately parabolic restoring potential. Because the radial component will be 
expanded about $v$, we posit the following form of $\phi(x)$

$$
\hat{\phi}(x)=\frac{v+\hat{b}(x)}{\sqrt{2}} \exp (i \hat{\theta}(x) / v)
$$

which leads to the Lagrangian density (up to second order)

$$
\hat{\mathscr{L}}_{G}=\frac{1}{2} \partial_{\mu} \hat{h} \partial^{\mu} \hat{b}-\mu^{2} \hat{b}^{2}+\frac{1}{2} \partial_{\mu} \hat{\theta} \partial^{\mu} \hat{\theta}+\mu^{4} / \lambda+O\left(\hat{\theta}^{3}, \hat{b}^{3}\right)
$$

The $\mu^{2} \hat{b}^{2}$ term indicates that the $\hat{b}$ mode has a mass $\sqrt{2} \mu$. Yet the $\hat{\theta}$ mode has no corresponding mass term, indicating it is a massless boson, known as the Goldstone boson. This is logical considering that there is no restoring force in the $\theta$ direction.

This shows how the addition of a complex scalar field $\phi$ with nonzero vev breaks an internal symmetry, giving itself mass. Goldstone's Theorem [8] requires there be a massless mode as well. We now go on to the $\mathrm{SU}(2) \times \mathrm{U}(1)$ symmetry of the SM.

\subsubsection{Higgs Mechanism}

Following the same prescription as the Goldstone case, we introduce a complex scalar field isospin doublet

$$
\phi=\left(\begin{array}{c}
\phi^{+} \\
\phi^{0}
\end{array}\right)
$$

the electroweak sector Lagrangian excluding fermions is therefore

$$
\begin{aligned}
\mathscr{L}_{\phi}= & \left(D_{\mu} \phi\right)^{\dagger}\left(D^{\mu} \phi\right)+\mu^{2}\left(\phi^{\dagger} \phi\right)-\frac{\lambda}{4}\left(\phi^{\dagger} \phi\right)^{2} \\
& -\frac{1}{4} W_{\mu \nu} W^{\mu \nu}-B_{\mu \nu} B^{\mu \nu}
\end{aligned}
$$

Again, the minimum is at $\left(\hat{\phi}^{\dagger} \hat{\phi}\right)=-\mu^{2} / 2 \lambda$, and we expand about the minimum, using the same form as before, adjusted for $\mathrm{SU}(2)$ symmetry

$$
\phi(x)=\exp \left(\frac{i \xi(x) \cdot \tau}{2 v}\right)\left(\begin{array}{c}
0 \\
(v+H(x)) / \sqrt{2}
\end{array}\right)
$$


The fact that the $\mathrm{SU}(2)$ is a local symmetry allows us to gauge transform such that the $\xi(x)$ fields vanish (unitary gauge). Thus the $\phi(x)$ field is

$$
\phi(x)=\left(\begin{array}{c}
0 \\
(v+H(x)) / \sqrt{2}
\end{array}\right)
$$

Substituting $\phi$ into $2.20[8]$ as

$$
\begin{aligned}
\mathscr{L}_{\phi}= & \frac{1}{2}\left(\partial_{\mu} H \partial^{\mu} H\right)-\mu^{2} H^{2} \\
& -\frac{1}{4}\left(\partial_{\mu} W_{1 \nu}-\partial_{\nu} W_{1 \mu}\right)\left(\partial^{\mu} W_{1}^{\nu}-\partial^{\nu} W_{1}^{\mu}\right)+\frac{1}{8} g^{2} v^{2} W_{1 \mu} W_{1}^{\mu} \\
& -\frac{1}{4}\left(\partial_{\mu} W_{2 \nu}-\partial_{\nu} W_{2 \mu}\right)\left(\partial^{\mu} W_{2}^{\nu}-\partial^{\nu} W_{2}^{\mu}\right)+\frac{1}{8} g^{2} v^{2} W_{2 \mu} W_{2}^{\mu} \\
& -\frac{1}{4}\left(\partial_{\mu} W_{3 \nu}-\partial_{\nu} W_{3 \mu}\right)\left(\partial^{\mu} W_{3}^{\nu}-\partial^{\nu} W_{3}^{\mu}\right)-\frac{1}{4} B_{\mu \nu} B^{\mu \nu} \\
& +\frac{1}{8} v^{2}\left(g W_{3 \mu}-g^{\prime} B_{\mu}\right)\left(g W_{3}^{\mu}-g^{\prime} B^{\mu}\right)+\cdots
\end{aligned}
$$

including only terms to second order in fields, which excludes $W W H, W W W$ and other known SM interactions.

The first two components of the $W$ field have quadratic terms. This indicates the fields are massive with mass $M_{W}=g v / 2$. The third component of the $W$ field mixes with the $B$ field. We can choose an orthogonal combination that mixes the two by an angle $\theta_{W}$ as in equation 2.10. Combined with the ladder $W$ operators instead of isospin 
components 1 and 2, and equations 2.11-2.13, we get

$$
\begin{aligned}
\mathscr{L}_{\phi}= & \frac{1}{2}\left(\partial_{\mu} H \partial^{\mu} H\right)-\mu^{2} H^{2} \\
& -\frac{1}{4}\left(\partial_{\mu} W_{\nu}^{+}-\partial_{\nu} W_{\mu}^{+}\right)\left(\partial^{\mu} W^{+\nu}-\partial^{\nu} W^{+\mu}\right)+\frac{1}{8} g^{2} v^{2} W_{\mu}^{+} W^{+\mu} \\
& -\frac{1}{4}\left(\partial_{\mu} W_{\nu}^{-}-\partial_{\nu} W_{\mu}^{-}\right)\left(\partial^{\mu} W^{-\nu}-\partial^{\nu} W^{-\mu}\right)+\frac{1}{8} g^{2} v^{2} W_{\mu}^{-} W^{-\mu} \\
& -\frac{1}{4}\left(\partial_{\mu} Z_{\nu}-\partial_{\nu} Z_{\mu}\right)\left(\partial^{\mu} Z^{\nu}-\partial^{\nu} Z^{\mu}\right)+\frac{1}{8}\left(g^{2}+g^{2}\right) v^{2} Z_{\mu} Z^{\mu} \\
& -\frac{1}{4}\left(\partial_{\mu} A_{\nu}-\partial_{\nu} A_{\mu}\right)\left(\partial^{\mu} A^{\nu}-\partial^{\nu} A^{\mu}\right)+\cdots
\end{aligned}
$$

which is the electroweak lagrangian without any fermion terms. In this case, it is apparent that there are four gauge bosons, three of which have mass, $H, W^{ \pm}, Z$, and one which is massless. The addition of the Higgs field also introduces mass terms for the fermions in the SM. That derivation will not be discussed here, in favor of deferring to a lucid explanation in Chapter 22 of [8] .

\subsection{Higgs Phenomenology}

The previous section focused on the tree level Standard Model theory. Now we must explore the specifics of higher order corrections as it applies to the search for the Higgs boson at the Tevatron.

\subsubsection{Indirect Constraints}

Partial wave scattering amplitudes for gauge boson pairs in the SM violate unitarity if the Higgs Boson mass exceeds $1 \mathrm{TeV} / \mathrm{c}^{2}[10,11]$. There is also a vacuum stability constraint [12] requiring the Higgs boson mass to be greater than $4 \mathrm{GeV} / \mathrm{c}^{2}$. Together these two constraints set a lower and upper bound on the Higgs boson mass within the confines of the SM. The constraints are believed to be related to the level of the $\Lambda_{\mathrm{GUT}}$, where the SM 


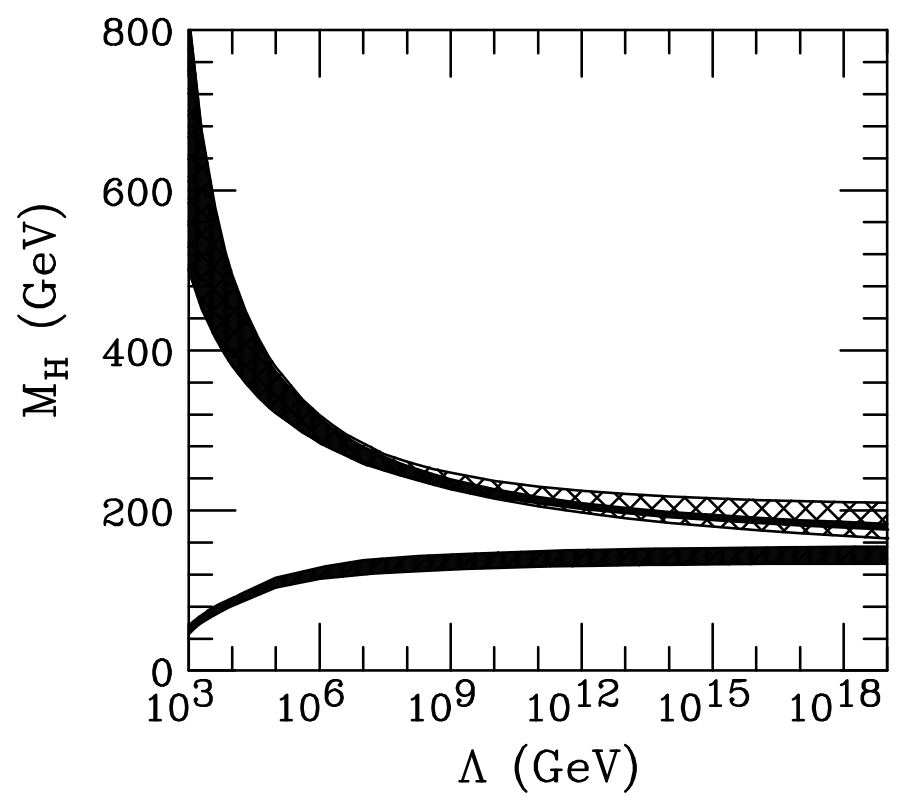

FIGURE 2.9: Theoretical Constraints on Higgs Mass [12] [13]

breaks down and we expect new physics. Figure 2.9 relates the two constraints and $\Lambda_{\mathrm{GUT}}$.

The SM also provides constraints on the Higgs boson mass from the measured top quark and $W$ boson masses. The current world average of the $W$ Boson mass is $80.398 \pm$ $0.025 \mathrm{GeV} / \mathrm{c}^{2}$ [6]. The top quark mass average is $173.1 \pm 1.6 \mathrm{GeV} / \mathrm{c}^{2}$ [14].

Figure 2.12 and Figure 2.13 show that all the measured SM parameters combined with the direct exclusion from LEP favor a Higgs boson between $114 \mathrm{GeV} / \mathrm{c}^{2}$ and 140 $\mathrm{GeV} / \mathrm{c}^{2}$. This happens to be the region where the $\mathrm{ZH}$ associated production process is the most sensitive.

\subsubsection{Tevatron Higgs Production}

Figure 2.14 summarizes the cross sections for different production mechanisms at $\sqrt{s}=$ $1.96 \mathrm{TeV}$ at the Tevatron.

The four dominant production processes are given in Figure 2.14, and the Higgs 


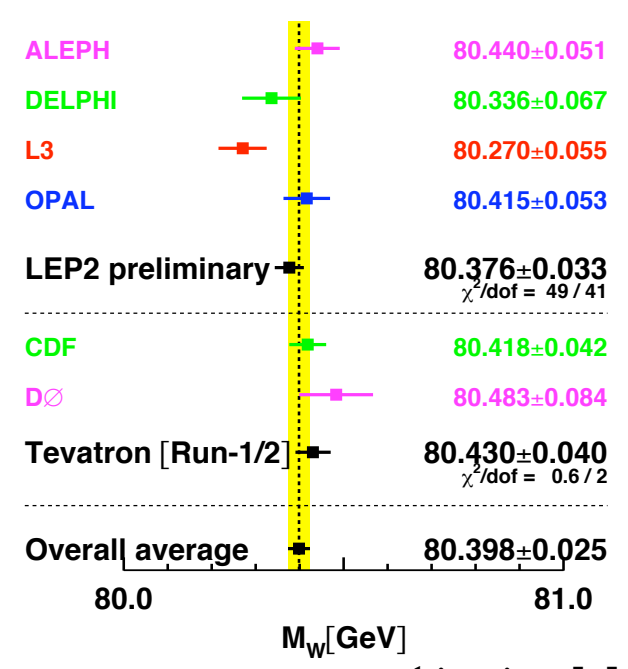

FIGURE 2.10: $M_{W}$ combination [6]

branching ratios are given as a function of mass in Figure 2.16. The production of Higgs through gluon-gluon fusion is predicted to have the largest cross section, but with a final state of two quarks (most likely $b \bar{b}$ ) at lower Higgs masses, it is completely swamped by QCD background. The next largest cross section is associated WH production, which is the subject of other searches at the Tevatron. This dissertation focuses on associated $Z H$

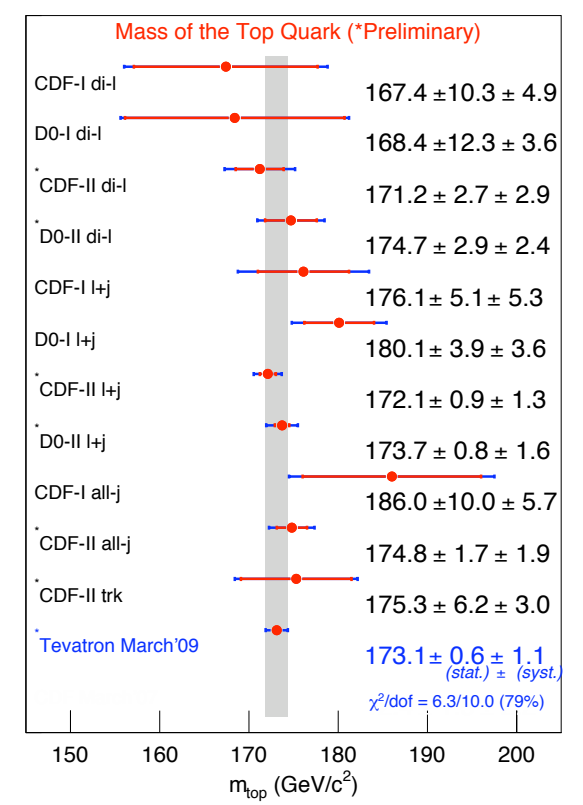

FIGURE 2.11: $m_{\text {top }}$ combination [14] 


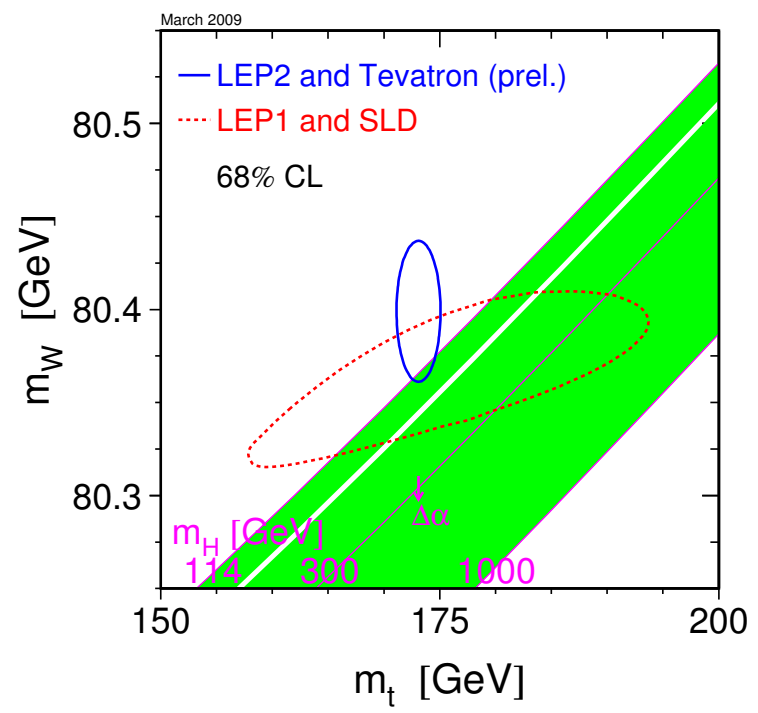

FIGURE 2.12: Summary of Electroweak Constraints on the Higgs [15] [16]. The blue ellipse is the $68 \%$ confidence interval centered around the current best measurements of $m_{W}$ and $m_{t}$, and favors a Higgs mass below $150 \mathrm{GeV} / \mathrm{c}^{2}$. Direct exclusion from LEP excludes the Higgs below $114 \mathrm{GeV} / \mathrm{c}^{2}$ at $95 \%$ C.L.

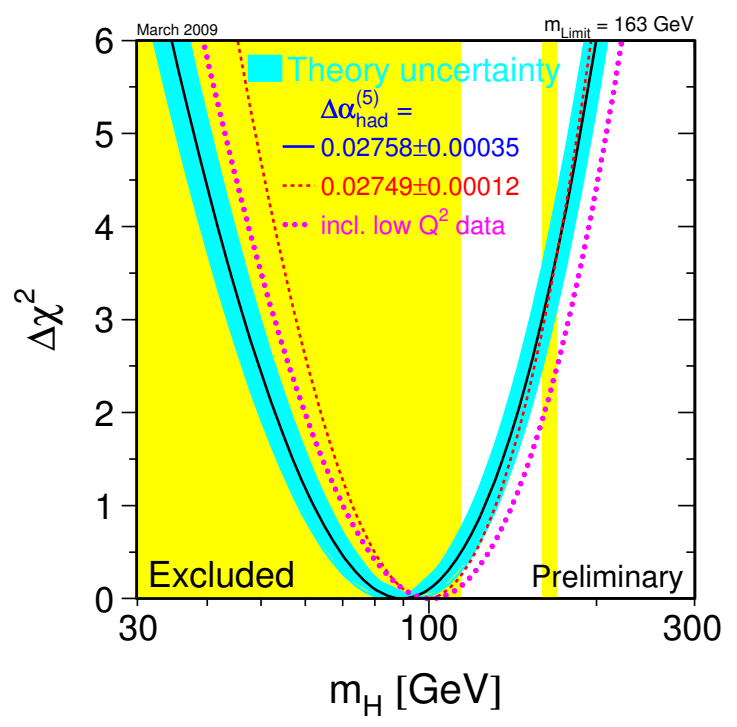

FIGURE 2.13: Global Fit to electroweak data [15] [16]. Excluded regions are shown in yellow, but here too we see a preference for a low mass Higgs boson 
SM Higgs production

\begin{tabular}{cc}
\hline$M_{H}$ & $q \bar{q} \rightarrow Z H(\mathrm{fb})$ \\
\hline 100 & 169.0 \\
105 & 145.0 \\
110 & 125.0 \\
115 & 107.9 \\
120 & 93.7 \\
125 & 81.6 \\
130 & 71.2 \\
135 & 62.4 \\
140 & 54.8 \\
145 & 48.2 \\
150 & 37.6 \\
\hline
\end{tabular}

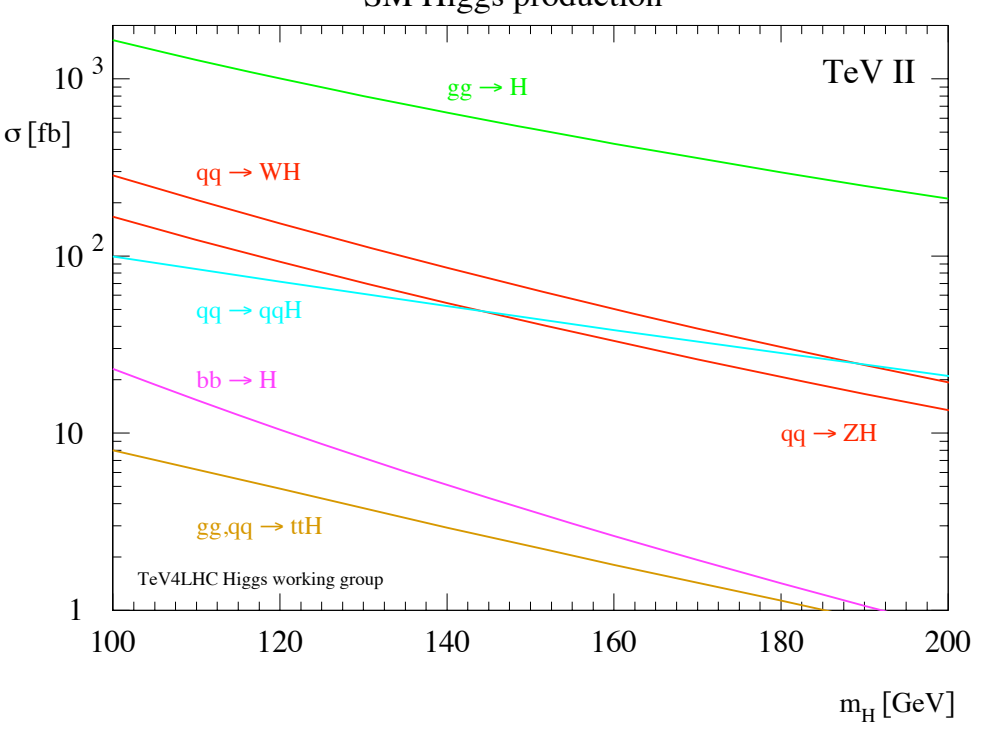

Figure 2.14: $p \bar{p} \rightarrow Z H$ Production Cross Section [17]

production via Higgs-strahlung, as shown in the third diagram of Figure 2.15.

The $Z H$ state consists of two heavy bosons. Since both couple to quarks and to leptons, they can both decay hadronically or leptonically. Higgs decays to a particle are proportional to the coupling between $H$ and the particle. As the heaviest particle accessible for Higgs boson masses below twice the $W$ boson mass, the $H$ preferentially decays to $b \bar{b}$, as in Figure 2.16. The $Z$ predominately decays hadronically, but does decay to $e^{+} e^{-}$and $\mu^{+} \mu^{-}$a total of $7 \%$ of the time. The diagram is Figure 2.17. The $p \bar{p} \rightarrow Z H \rightarrow l \bar{l} b \bar{b}$ process has a low cross section, but the requirement of a dilepton signature from the $Z$ greatly reduces QCD background. 


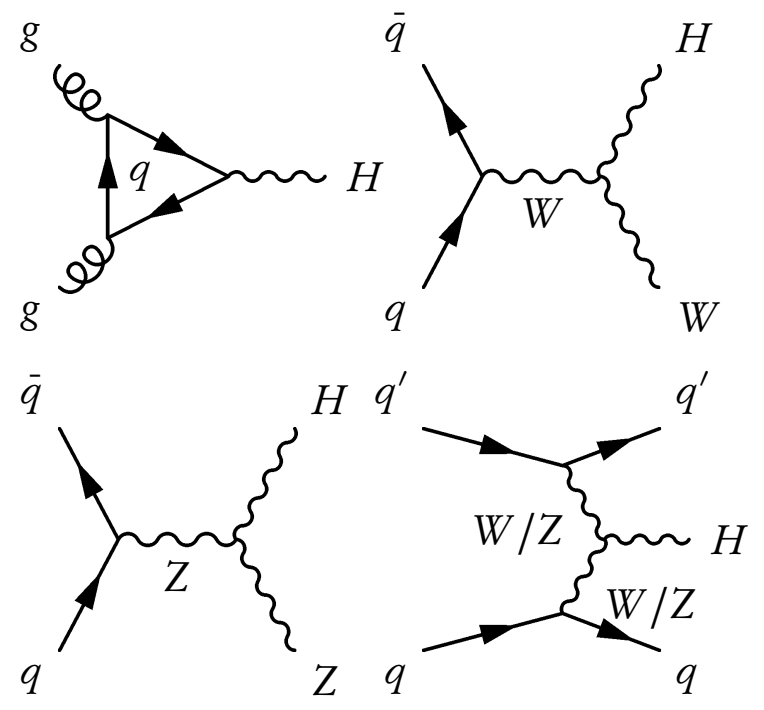

FIGURE 2.15: Dominant Higgs production processes at the Tevatron [18]. The subject of this dissertation is the lower left diagram.

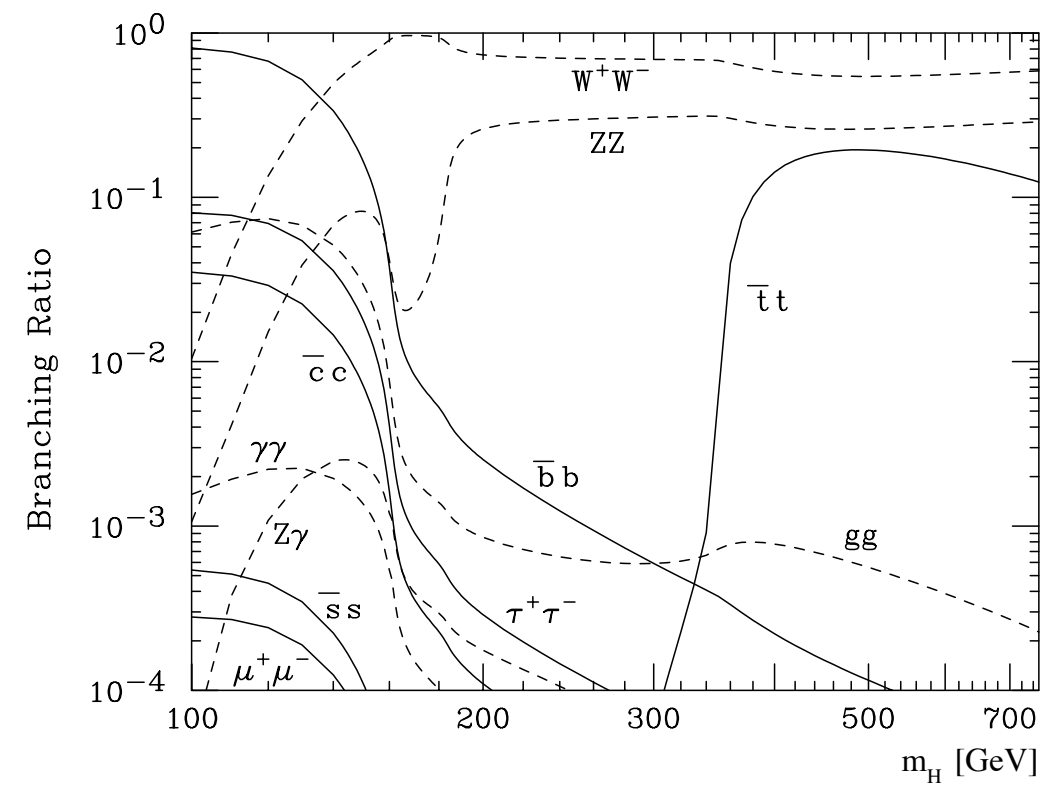

FIGURE 2.16: Higgs Boson Branching Ratios [6] 


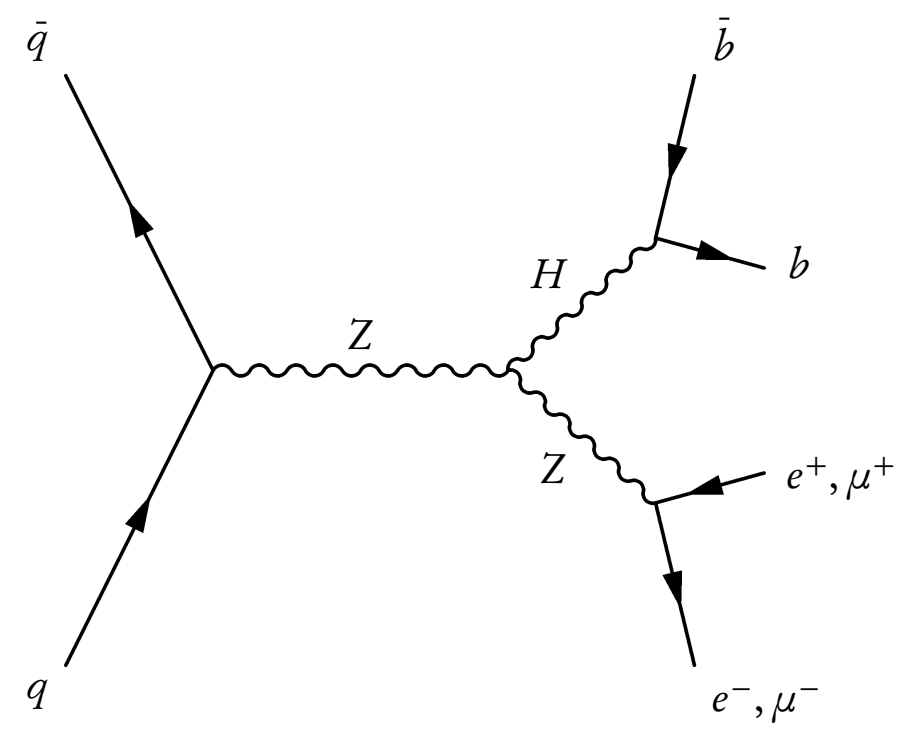

FigURE 2.17: $\mathrm{ZH} \rightarrow l^{+} l^{-} b \bar{b}$ production mechanism 


\section{Experimental Apparatus}

Since the 1940s, the study of particle physics has required collisions at higher and higher particle energies. The latest operational particle accelerator is the Tevatron, located at Fermi National Accelerator Lab (FNAL) in Batavia, Illinois. Originally constructed in the 1980s, the Tevatron was upgraded in 1999 to achieve a center of mass collision energy of $\sqrt{s}=1.96 \mathrm{TeV}$. This makes the Tevatron the highest energy hadron collider currently operational. The colliding particles are protons $(p)$ and anti-protons $(\bar{p})$, and through a multi-stage process, the Tevatron accelerates them to a speed of $\beta=0.999999$, or one part per million away from the speed of light.

The Large Hadron Collider (LHC) is due to come online in late 2009. Instead of a $p, \bar{p}$ collider like the Tevatron, the LHC is a $p, p$ collider. It has a higher energy $(\sqrt{s}=14 T e V)$, but the Higgs production mechanism is expected to be dominated by gluon-gluon fusion, which favors high mass $\left(150 \mathrm{GeV} / \mathrm{c}^{2}<m_{H}<190 \mathrm{GeV} / \mathrm{c}^{2}\right) \mathrm{Higgs}$ searches. 


\subsection{Tevatron}

The Tevatron is a $6.3 \mathrm{~km}$ circumference circular accelerator that collides $p$ and $\bar{p}$, shown in Figure 3.1. Hydrogen gas is separated into constituent atoms, which are then ionized into $H^{-}$. These ions enter a Crockcroft-Walton pre-accelerator [19], which applies an electric field to the ions and accelerates them to $750 \mathrm{keV}$. The ions then enter the linear accelerator (Linac), which uses $\mathrm{RF}$ waves to further increase the energy to $400 \mathrm{MeV}$. The electrons are stripped from the $H^{-}$ions, leaving protons, which are injected into the booster. The booster is a synchrotron with a $0.5 \mathrm{~km}$ circumference, and it accelerates the protons to $8 \mathrm{GeV}$. These protons are then injected into Main Injector, a larger synchrotron $3 \mathrm{~km}$ in circumferance.

\section{FERMILAB'S ACCELERATOR CHAIN}

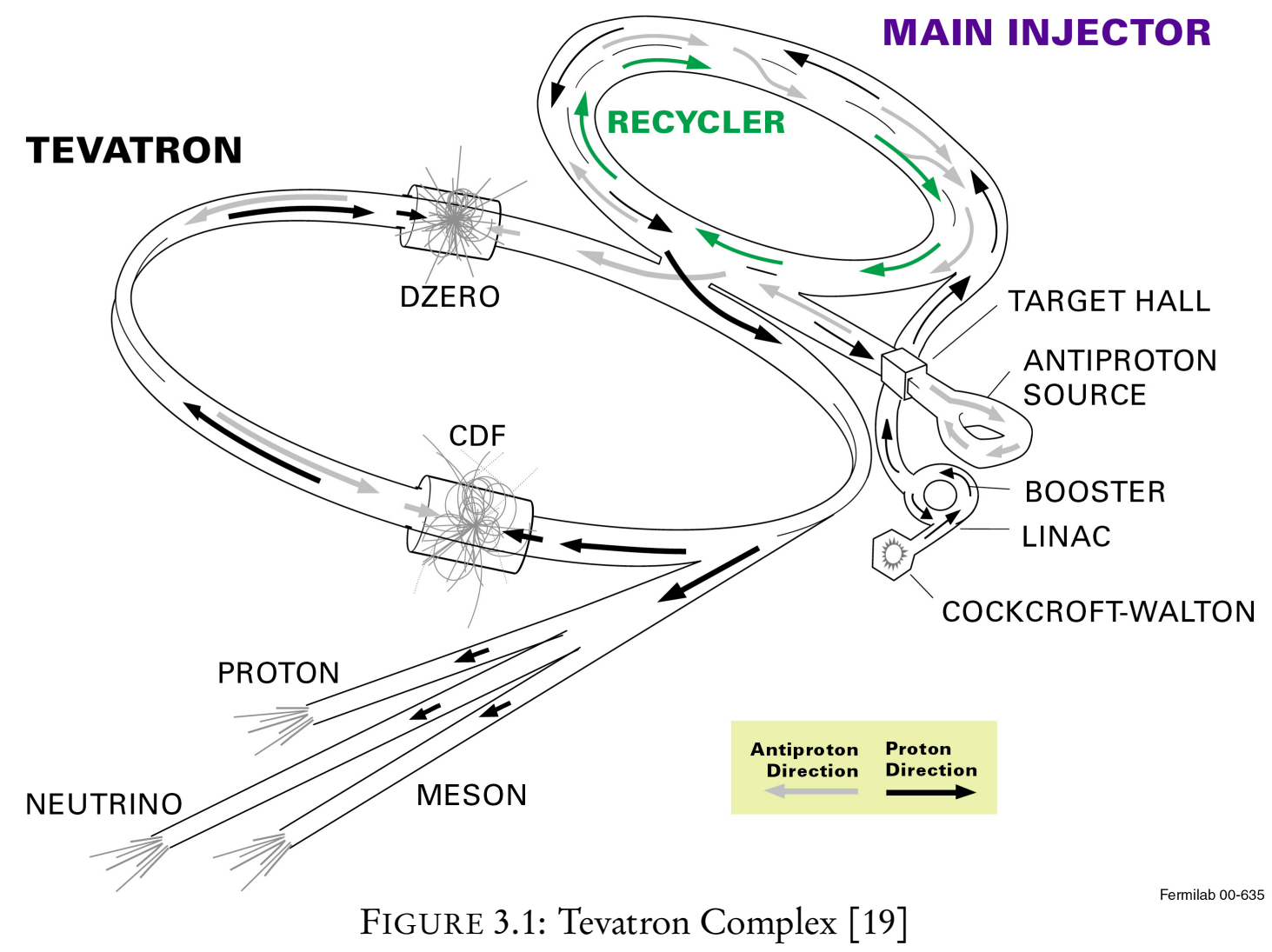


The main injector performs several functions, although not concurrently. First, it accelerates the protons from the booster to $150 \mathrm{GeV}$, and injects them into the Tevatron. Second, for $\bar{p}$ production, it accelerates protons to $120 \mathrm{GeV}$, which are then directed into a nickel target, which produces a large number of particles, including the constituents for $\bar{p}$, which are directed toward the anti-proton source. Third, it is used to collect $\bar{p}$ produced by the anti-proton source, and accelerating them to $150 \mathrm{GeV}$ as well, for injection into the Tevatron.

Many different types of particles are produced when the nickel target is struck by 120 $\mathrm{GeV}$ protons. The anti-protons are separated out by magnetic spectroscopy, and are then moved to the Debuncher. Since the anti-protons have a very large spread in momentum, the Debuncher reduces the momentum spread. The anti-protons are then moved to the Accumulator, which stores anti-protons until they are ready for injection, at which time they are moved to the main injector, accelerated to $150 \mathrm{GeV}$ and then injected into the Tevatron. Producing anti-protons in this manner is difficult, and is the limiting factor in instantaneous luminosity at the Tevatron.

The Recycler, which shares a tunnel with the Main Injector, was originally conceived to store anti-protons between physics runs. This proved unfeasible, but the Recycler turned out to be useful as an additional place to store anti-protons besides the Accumulator. This greatly increases the anti-protons available during a physics run, and consequently greatly increases the instantaneous luminosity of the Tevatron.

The main Tevatron ring accepts protons and anti-protons at an energy of $150 \mathrm{GeV}$ from the Main Injector. They are accelerated using superconducting 4.2 $T$ magnets in the $6.3 \mathrm{~km}$ circumference ring to $0.98 \mathrm{TeV}$, making the center of mass energy $\sqrt{\mathrm{s}}=1.96$ $\mathrm{TeV}$. The $p$ and $\bar{p}$ beams travel in opposite directions around the ring, and cross at the two detectors, the Collider Detector at Fermilab (CDF) and DØ detectors.

If the $p$ and $\bar{p}$ were equally distributed throughout the ring, the chance of a collision at any given time would be very low because of a lack of sufficient densities. To remedy this, 
the particles are distributed into bunches, about $50 \mathrm{~cm}$ long, containing approximately $10^{11} p$ and $10^{10} \bar{p}$. There are 36 bunches of both $p$ and $\bar{p}$ located around the ring, and they cross every $396 n s$ [6]. Bunching greatly increases instantaneous luminosity, which is given by the formula

$$
\mathscr{L}=\frac{N_{B} N_{p} N_{\bar{p}} f}{2 \pi \sigma_{p} \sigma_{\bar{p}}}
$$

where $N_{B}$ is the number of bunches in the ring, $N_{p}\left(N_{\bar{p}}\right)$ are the number of protons (antiprotons) per bunch, $f$ is the bunch revolution frequency, and $\sigma_{p}\left(\sigma_{\bar{p}}\right)$ is the effective width of the proton (anti-proton) bunches. The integrated luminosity (in units of $\mathrm{pb}^{-1}$ ), when combined with the cross section (in $\mathrm{pb}$ ) gives the number of collisions that occurred. Since the beginning of Run II in March 2001, the integrated luminosity is shown in Figure 3.2. This analysis uses $2.7 \mathrm{fb}^{-1}$ of CDF II data, through period 17.

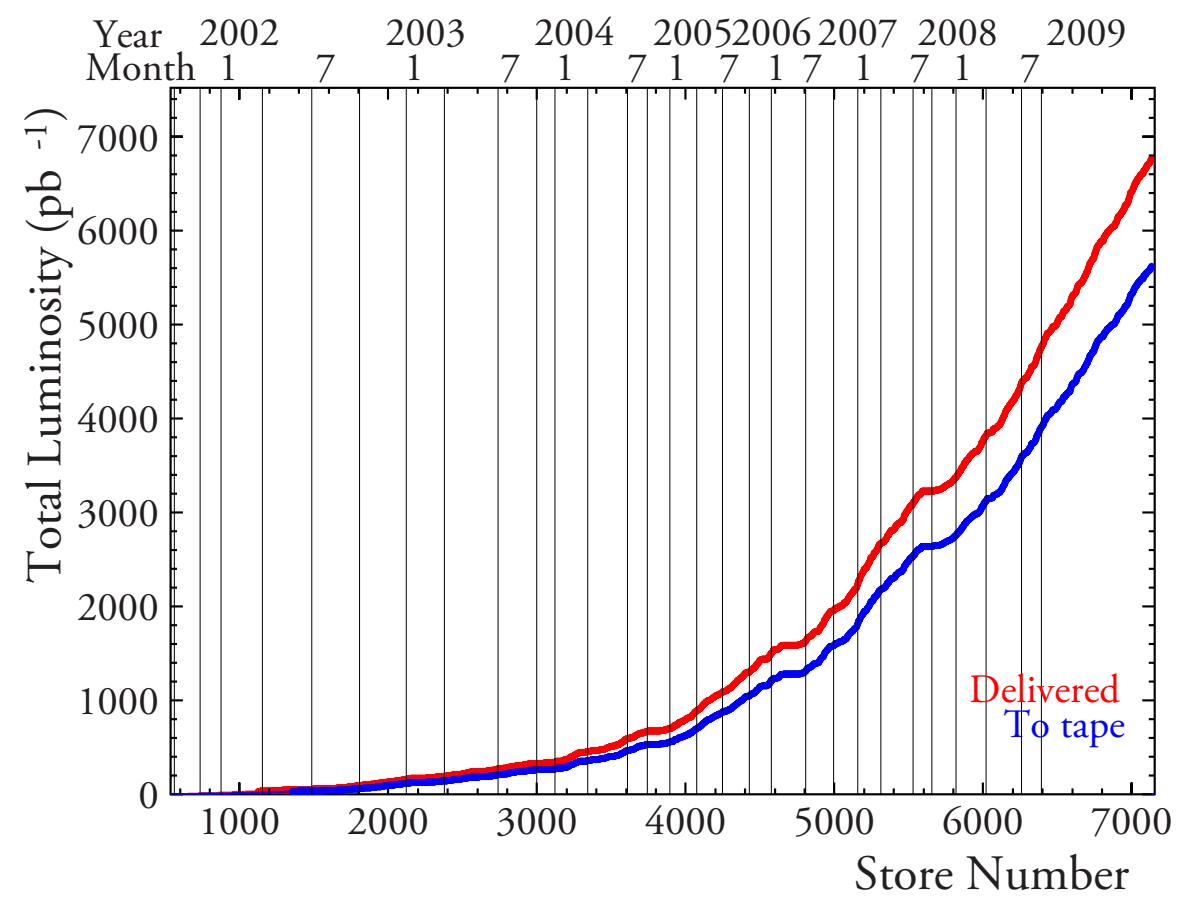

FIGURE 3.2: Integrated luminosity at CDF II 


\subsection{CDF II Detector}

The CDF detector is a general purpose, azimuthally and forward-backward symmetric detector at the Tevatron. The $z$ axis is taken to be along the proton beam direction, and the $\phi$ is defined with respect to the outward direction on the Tevatron ring. Because the cylindrical coordinate is not invariant under Lorentz transforms, the pseudo-rapidity is used, defined as $\eta=-\ln \tan (\theta / 2)$, which is additive under Lorentz boosts in the beam direction in the massless approximation. Together, $z, \phi$, and $\eta$ form the coordinate system for the CDF detector. Distance between two objects is defined as $\Delta R=\sqrt{(\Delta \phi)^{2}+(\Delta \eta)^{2}}$ A schematic of the detector is shown in Figure $3.3[20,21]$.

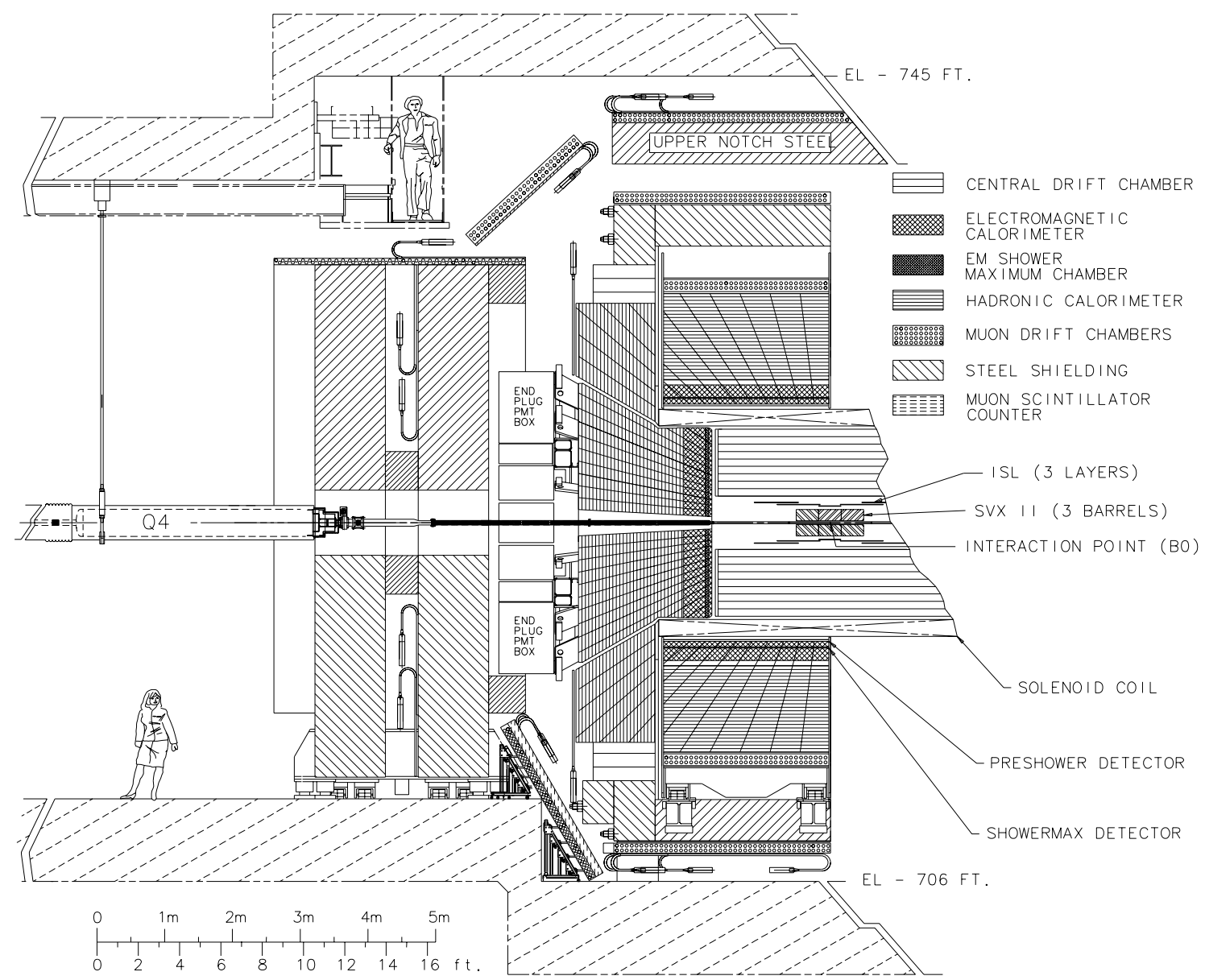

FIGURE 3.3: CDF detector schematic [20, 21] 
In order of inward to outward from the beam pipe, the CDF detector has the following parts:

- Cerenkov Luminosity Counter (CLC) that measures instantaneous luminosity.

- Silicon Vertex Detector (SVX) that provides tracking of charged particles near the interaction point.

- Central Outer Tracker (COT) drift chamber consisting of drift wires in a strong (1.4 T) magnetic field to measure momenta and charge.

- Electromagnetic calorimeter to measure energy deposition from objects like electrons and photons.

- Hadronic calorimeter to measure energy deposition from protons, pions and other hadrons.

- Muon detector measures muons, which are assumed to pass through the other detectors essentially unaffected.

All parts that make up the tracking volume are shown in Figure 3.4.

\subsubsection{Cerenkov Luminosity Counter}

The Cerenkov Luminosity Counter is used to measure instantaneous luminosity. It is placed very close to the beam line, at $3.7<|\eta|<4.7[20,22,23]$. Since deceleration of charged particles in matter produces Cerenkov radiation, the CLC measures the amount of Cerenkov radiation produced, thereby measuring the number of $p$ and $\bar{p}$ in every bunch. The relation used is

$$
\mu f_{\mathrm{BC}}=\sigma_{\text {inelastic }} \mathscr{L}
$$

where $\mu$ is the average number of $p, \bar{p}$ interactions per bunch crossing, $f_{\mathrm{BC}}$ is the bunch crossing rate, and $\sigma_{\text {inelastic }}$ is the inelastic cross section of the beam. Knowing the other three variables allows us to solve for $\mathscr{L}$, the instantaneous luminosity. 


\section{CDF Tracking Volume}

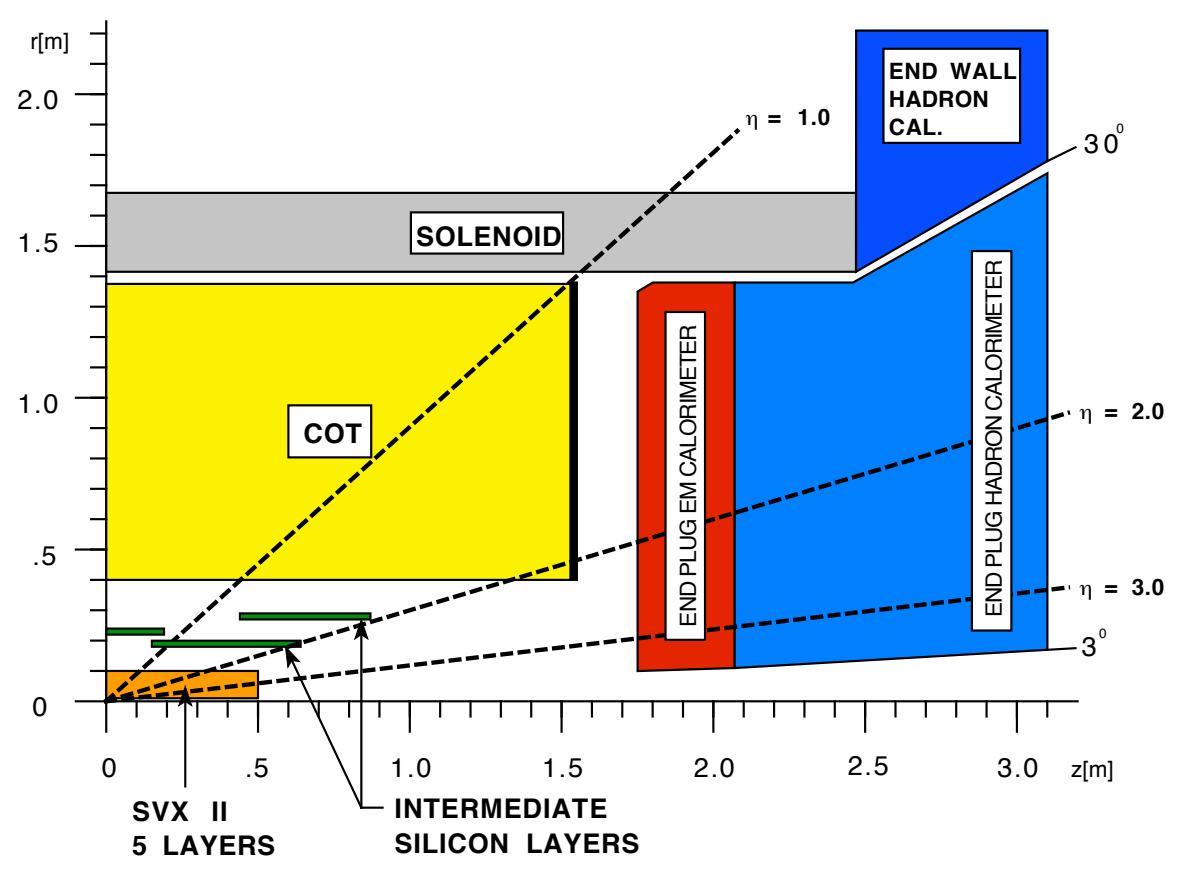

FIGURE 3.4: CDF Tracking Volume

\subsubsection{Silicon Tracking}

The silicon tracking system $[24,25]$ is located closest to the beam pipe and provides high precision measurement of position for particles from the collision. In the case of the $\mathrm{ZH}$ analysis, the $b$ quarks from the Higgs boson are measured using the silicon detector.

A voltage is applied across the silicon detector to strip out the excess electrons. When an ionizing particle from a collision passes through the silicon, it ionizes the silicon, causing a buildup of charge at the terminal, which is then measured. The position of the silicon sensor that generated the current allows the location of the particle to be inferred and the location of successive hits allows a path to be reconstructed for the particle.

The silicon detector is broken into the Silicon Vertex (SVXII) detector and the intermediate silicon layers (ISL). The SVXII detector has five concentric barrels, each of which has silicon sensors on the inner surface. Additionally, there is a silicon strip mounted directly on the beampipe, which is called Layer 00 (LO0). Outside the SVXII, 
the ISL is another cylindrical detector that extends silicon coverage to $|\eta|=2.0$, as shown in Figure 3.4. The barrel structure of the SVX detector is shown in Figure 3.5. The total SVX detector has 722,432 channels.

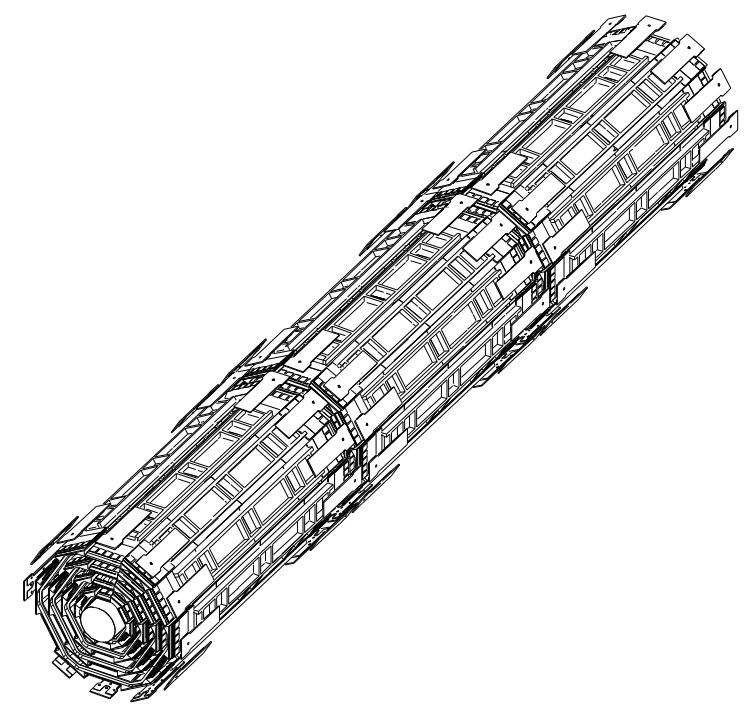

FIGURE 3.5: Silicon detector barrel structure

\subsubsection{Central Outer Tracker}

The Central Outer Tracker [26] (COT) is another, much larger tracking volume that extends from $0.43 m$ to $1.32 m$ radially and from $-1.5 m$ to $+1.5 \mathrm{~m}$ along the beam direction. The chamber is filled with a low pressure mixture of argon and ethane and contains 8 "superlayers", each of which contains 12 wire layers for a total of 96 layers. When a particle goes through the chamber, it ionizes the gas, leading to free electrons being attracted to the sensing wires which are then measured. The superlayers alternate as axial superlayers (measure $\phi$ ) and stereo superlayers (measure $z$ ). The fine resolution of the wires in the drift chamber allow a transverse momentum resolution of $0.15 \% \times p_{T} / G e V$. Combined with silicon, the resolution is $0.07 \% \times p_{T} / G e V$. In order to measure the the charge of a particle, the solenoid around the COT applies a 1.4 $T$ magnetic field, which causes the path of the particle to curl into a helix, allowing the charge and momentum to be inferred. 


\subsubsection{Calorimeters}

The electromagnetic and hadronic calorimeters sit outside the solenoid. They are divided into central $(|\eta|<1.1)[27,28]$ and plug $(1.1<|\eta|<3.6)$ [29]. The EM calorimeter absorbs energy from electrons and photons, whereas the hadronic calorimeter absorbs energy from particles not absorbed in the EM calorimeter (not including muons).

The calorimeters are composed of alternating layers of scintillator and metal. Particles passing through the calorimeter interact with the dense materials and produce a shower of particles which are detected in the photomultiplier tubes. The em calorimeter metal is lead and the hadronic calorimeter metal is iron. The towers are segmented into 15 degree wedges in $\phi$ and 0.1 in $\eta$. In the plug region, $\phi$ segmentation is 7.5 degree wedges until $\eta=2.11$, after which again the wedges in $\phi$ are 15 degrees. Together the scintillator and metal in the calorimeters form six interaction lengths of material, which would be expected to trap $99.8 \%$ of the energy of interacting particles passing through on average.

\subsubsection{Muon Detectors}

The muon detectors are located outside all other detectors because the muons are long lived and and do not strongly interact with the calorimeter. The detectors are single wire drift chambers, and cover most of the space where $|\eta|<1.0$. There are three parts to the muon detectors, the central muon detector (CMU), the central muon upgrade (CMP), and the central muon extension (CMX). The CMP is an additional layer of drift chamber outside the CMU. The coverage is shown in Figure 3.6. 


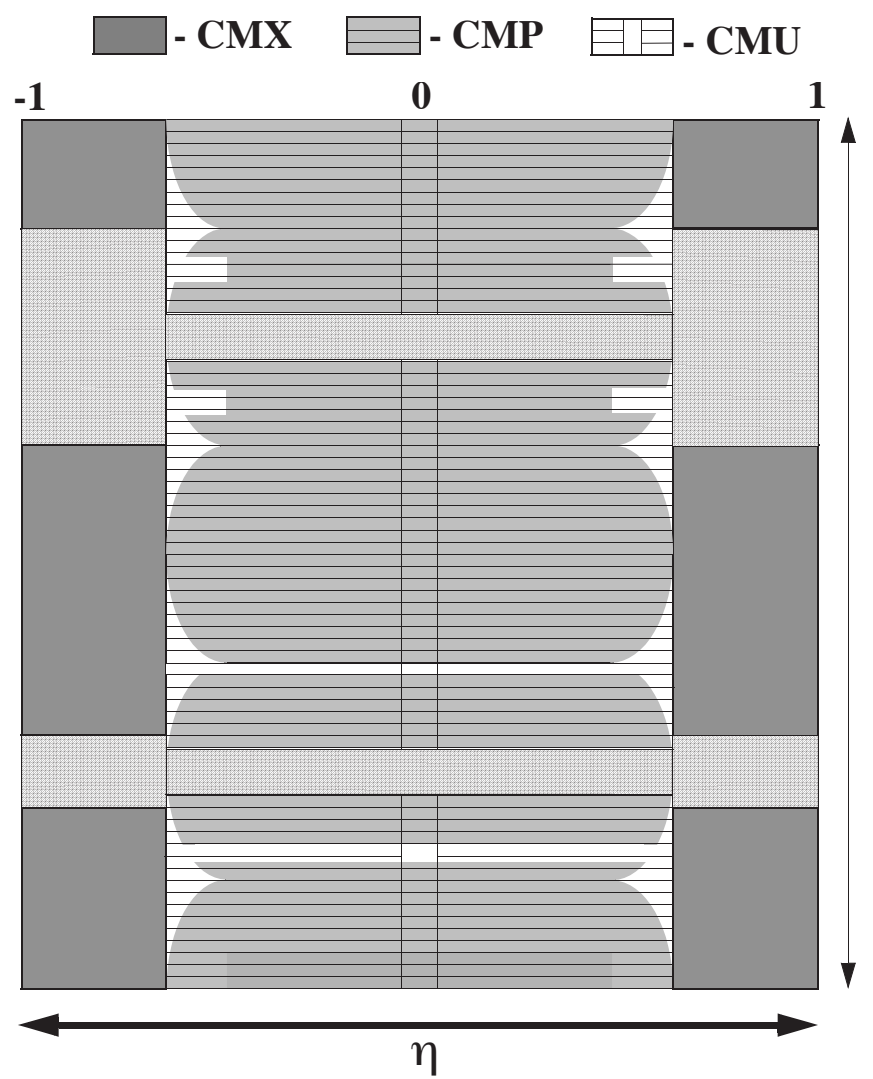

FIGURE 3.6: Muon detector coverage

\subsection{CDF Software}

The CDF detector is an amazingly complex machine, with many different interoperating systems. To extract maximum sensitivity for a physics analysis, all the components must function as a whole to reconstruct particles and trajectories from hits on wires. This requires a combination of hardware and software to isolate interesting events for analysis and reconstruct the particle content of the events.

\subsubsection{Event Triggers}

Collisions occur at a rate of approximately $2.5 \mathrm{MHz}$. Storing all the hits and raw detector level information for an event is approximately $200 \mathrm{~KB}$. That translates to a raw data rate of nearly $500 \mathrm{~GB} / \mathrm{s}$, which was well beyond the reach of computing when the CDF 
detector was constructed and upgraded. Coupled with the fact that most $p \bar{p}$ collisions result in some sort of soft scattering, which are not terribly interesting from a physics perspective, we know events must be discarded.

The system for deciding what events to keep is called triggering. At CDF, there are three levels of event triggers. Each one successively applies more stringent criteria. If an event passes all three triggers, it is recorded to a permanent storage medium.

The Level 1 trigger (L1) is purely hardware based and it uses calorimeter information, tracks reconstructed in the COT with the eXtremely Fast Tracker (XFT)[30] algorithm, and muon chamber stubs to make a decision. The specialized hardware nature of the trigger and simplistic algorithm ensures fast rejection, allowing the event rate to be reduced from approximately $2.5 \mathrm{MHz}$ to $20 \mathrm{kHz}$.

The Level 2 trigger (L2) has better (but slower) resolution and identification algorithms. It also considers tracking information from silicon, allowing better rejection of unwanted events. It reduces the event rate from $20 \mathrm{kHz}$ to $300 \mathrm{~Hz}$.

The Level 3 trigger (L3) is entirely in software and is performed by a computing cluster. It takes raw detector output, reconstructs higher level objects, clustered calorimeter energies, and sophisticated reconstructed tracking from both COT and silicon as inputs. It reduces the event rate from $300 \mathrm{~Hz}$ to $75 \mathrm{~Hz}$, after which the events are written to permanent storage.

\subsubsection{Lepton Identification}

The $\mathrm{ZH}$ analysis depends on accurate measurement of charged leptons, $e, \mu(\tau$ does not have sufficient acceptance to contribute to the result at this luminosity). These two charged leptons are relatively easy to measure in the CDF detector, as they leave tracks in the COT and do not shower like gluons and quarks. The following quantities are used in selection of electrons and muons (see chapter 4). 


\section{Electron Quantities}

- $E_{T}$ - Transverse energy - the energy deposited in the calorimeters. For electrons, at most two towers (neighboring in $\eta$ ) are allowed.

- $p_{T}$ - Transverse momentum - the momentum of the electron as measured in the COT tracking detector.

- Had/EM - The ratio of energy deposited in the hadronic to the energy deposited in the EM calorimeters. Electrons mostly deposit in the EM calorimeter, so this should be low $(<10 \%)$.

- $L_{s b r}$ - This indicates the agreement of the electron with the expected lateral shower profile.

- $|\Delta x|$ and $|\Delta z|$ - Separation between COT track position and EM shower position.

- $E / P$ - Ratio of calorimeter energy to momentum from the COT. May deviate from unity because of bremsstrahlung.

- Isolation - Ratio of energy deposited outside the center of an electron cluster to the total energy inside a $\Delta R=0.4$ cone, excluding the electron. Electrons from a $Z$ boson are isolated from jets and other leptons, so this quantity should be small [31].

- $\chi_{\text {strip }}^{2}$ - Comparison of shower profile to expected profile.

\section{Muon Quantities}

- $p_{T}$ - Transverse momentum - the momentum of the muon as measured in the COT tracking detector.

- $|\Delta x|$ and $|\Delta z|$ - Separation between COT track position and the stub seen in the muon chambers. 
- $E_{\text {em,hadronic }}$ must be low for a muon as they do not deposit much energy in the calorimeters.

- Isolation - Muon COT track must be relatively isolated from other tracks within a $\Delta R=0.4$ cone. Muons from a $Z$ boson decay are isolated from jets and other leptons.

\subsubsection{Jet Modeling and Reconstruction}

By the principle of confinement, QCD requires that objects with nonzero color charge cannot exist outside of a bound state. This means that in high energy collisions, any high $p_{T}$ quarks or gluons (which carry color charge) must hadronize, or turn into a set of hadrons that are colorless. To do this, the energy of the original quark or gluon causes pair production of new quark antiquark pairs from the vacuum. This process repeats until the newly produced items can form colorless bound states. Typically, a high energy quark hadronizes into a stream of 10-30 particles, collectively referred to as a jet.

In the CDF detector reconstruction, jets are found by the JetCLU [32] algorithm, which operates by iteratively calculating the centroid of energy deposition in the calorimeter towers until the centroid no longer changes. These raw jet energies are corrected to account for

- Nonuniform calorimeter response as a function of jet energy.

- $\eta$ corrections to account for gaps in the detector

- Multiple $p, \bar{p}$ interactions at a single bunch crossing, which can happen at high luminosities.

These lead to significant scale corrections for the measured jet energy, given in Figure 3.7. The uncertainty on the jet energy scale is given in Figure 3.8. Since the systematic on 


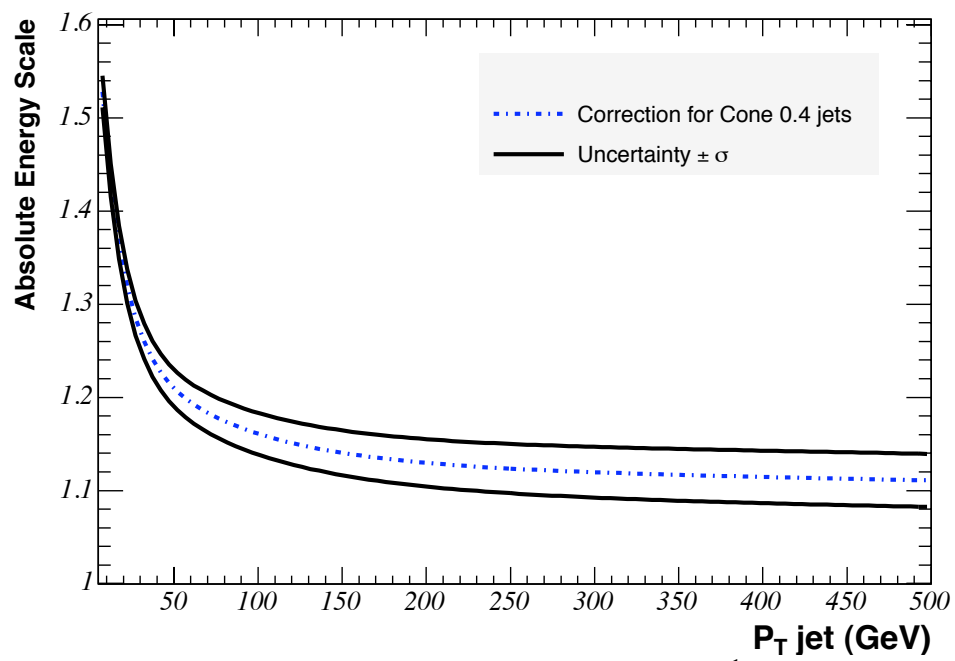

FIGURE 3.7: Jet energy scale

the jet energy scale will affect different processes differently, this systematic must be propagated forward in the analysis. This is examined in chapter 6.

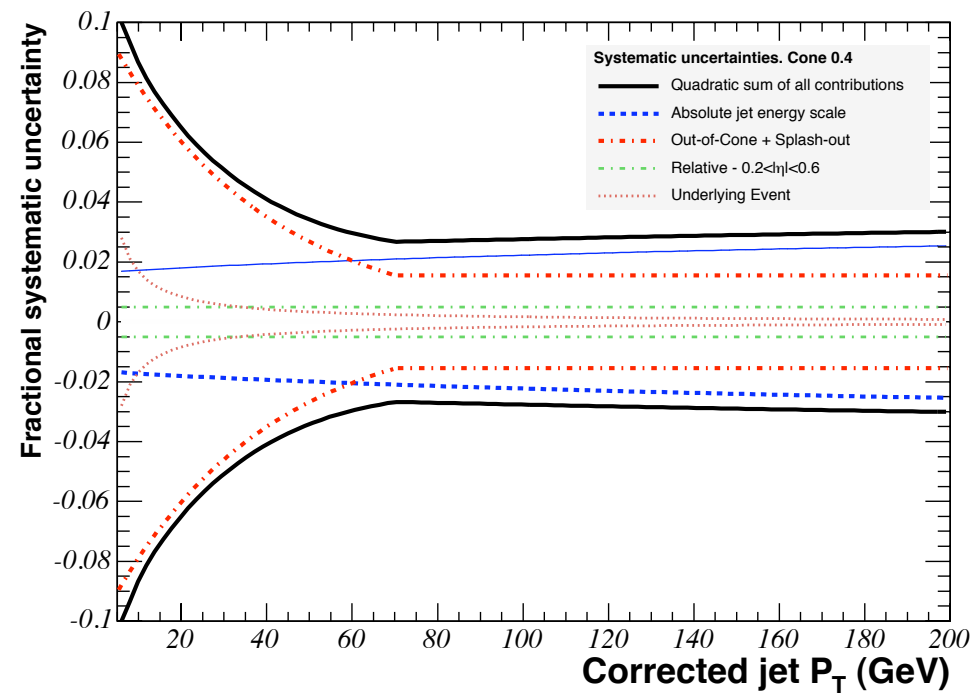

FIGURE 3.8: Jet energy scale systematic uncertainty 


\subsubsection{SecVtx Tagging}

$b$ quarks decay through the weak force, which requires they have long lifetimes compared to particles that decay through the strong force. The high resolution particle tracking available from the silicon vertex (SVX) detector allows detection of production of the $b$ quark at the primary interaction vertex, a short decay distance $(\sim \mathscr{O}(1 \mathrm{~mm}))$ and a secondary decay vertex to other particles. The SECVTX (Secondary Vertex) algorithm looks for a decay vertex displaced from the collision vertex using the silicon detector. Tracks where this displacement is found are then matched with jets within a $\Delta R=0.4$ cone, allowing a jet to be tagged as coming from a $b$ quark. Two different sets of criteria (loose and tight) are possible, and both are used in the $\mathrm{ZH}$ analysis (see chapter 4). Approximately $45 \%$ of $b$ quarks meet loose criteria and $35 \%$ of $b$ quarks meet tight criteria at a jet $E_{T}$ of $30 \mathrm{GeV}$. 


\section{Event Selection}

We select $q \bar{q} \rightarrow Z H \rightarrow l^{+} l^{-} b \bar{b}$ candidates by requiring (i) high- $p_{T}$ lepton trigger, (ii) two leptons each with $p_{T}>20 \mathrm{GeV} / \mathrm{c}$ and a dilepton invariant mass in the $\mathrm{Z}$ mass window, $76 \mathrm{GeV} / \mathrm{c}^{2}<M_{l l}<106 \mathrm{GeV} / \mathrm{c}^{2}$ (iii) two cone-0.4 jets with $E_{T}>15 \mathrm{GeV}$, one of which has $E_{T}>25 \mathrm{GeV} / \mathrm{c}^{2}$, and (iv) 2 loose SECVTX $b$-tags or exclusively 1 tight SECVTX $b$-tag. These requirements are summarized in Table 4.1.

This selection was designed for the $\mathrm{ZH} \rightarrow l^{+} l^{-} b \bar{b}$ neural network analysis at CDF. Results with $1 \mathrm{fb}^{-1}$ of data using the same selection are published in [33]. A description of selection and trigger requirements follows.

A central lepton trigger

One high $E_{T}$ central lepton with 'tight' requirements A second high $E_{T}$ lepton of the same flavor with the opposite sign $\mathrm{Z}$ boson mass window of $76 \mathrm{GeV} / \mathrm{c}^{2}$ to $106 \mathrm{GeV} / \mathrm{c}^{2}$ Two or more jets with $E_{T}>15 \mathrm{Ge} V$ and $|\eta|<2$

One or more jets with $E_{T}>25 \mathrm{GeV}$ Either: 2 'loose' $b$-tags or 1 'tight' $b$-tag

Table 4.1: Summary of Event Criteria 


\subsection{Event Criteria}

In $p \bar{p}$ collisions, the interacting particles are the quarks and gluons that compose the protons. Since the coupling of quarks to the strong force is much higher than the coupling to the electroweak force, the dominant product of interaction is hadronic. Since we seek to reduce this large hadronic background, we search for a high $p_{T}$ lepton using the CDF central high $p_{T}$ lepton trigger [34]. These are summarized in Tables 4.2 and 4.3.

\begin{tabular}{c|c} 
Trigger & Central Electron \\
\hline Level 1 & Central Calorimeter $E_{T} \geq 8 \mathrm{GeV}$ \\
& XFT $p_{T} \geq 8.34 \mathrm{GeV} / \mathrm{c}$ \\
& $\mathrm{Had} / \mathrm{EM}<0.125$ \\
\hline Level 2 & $\mathrm{L} 2 E_{T} \geq 16 \mathrm{GeV}$ \\
& $\mathrm{L} 2 \mathrm{Had} / \mathrm{EM} \leq 0.125$ \\
& $|\eta|<1.317$ \\
\hline Level 3 & Central $E_{T}>18 \mathrm{GeV}$ \\
& Central Had/EM $<0.125$ \\
& Central $\Delta z \leq 2 \mathrm{~cm}$ \\
& Track $p_{T}>9 \mathrm{GeV} / \mathrm{c}$ \\
\hline
\end{tabular}

Table 4.2: Central electron trigger path

\begin{tabular}{c|c|c} 
Trigger & CMUP & CMX \\
\hline Level 1 & CMU stub $p_{T} \geq 6 \mathrm{GeV} / \mathrm{c}$ & $\mathrm{CMX} \mathrm{stub} p_{T} \geq 6 \mathrm{GeV} / \mathrm{c}$ \\
& $\mathrm{XFT} p_{T} \geq 4.09 \mathrm{GeV} / \mathrm{c}$ & $\mathrm{XFT} p_{T} \geq 8.34 \mathrm{GeV} / \mathrm{c}$ \\
& $\mathrm{CMP} \mathrm{Stub}$ & \\
\hline Level 2 & $\mathrm{XFT} p_{T} \geq 14.77 \mathrm{GeV} / \mathrm{c}$ & $\mathrm{XFT} p_{T} \geq 14.77 \mathrm{GeV} / \mathrm{c}$ \\
\hline Level 3 & $p_{T} \geq 18.0 \mathrm{GeV} / \mathrm{c}$ & $p_{T} \geq 18.0 \mathrm{GeV} / \mathrm{c}$ \\
& $\mathrm{CMP} \Delta x<20$ & $\mathrm{CMX} \Delta x<10$ \\
& $\mathrm{CMU} \Delta x<10$ & \\
\hline
\end{tabular}

Table 4.3: Central muon trigger paths

The central electron trigger looks for energy in an EM calorimeter tower with a corresponding XFT track. At level 2, there must be a EM tower with $E_{T}>8 \mathrm{GeV}$ and nearby towers must sum up to $E_{T}>16 \mathrm{GeV}$. At level 3, there must be a corresponding track with $p_{T}>9 \mathrm{GeV} / \mathrm{c}$ and $\Delta z \leq 2 \mathrm{~cm}$. The minimum $E_{T}$ is $18 \mathrm{GeV}$, but because of trigger turn on effects, we only consider electrons above $20 \mathrm{GeV}$. 
Muons have two triggers, CMUP and CMX, corresponding to the parts of the muon detector. The CMUP trigger requires aligned muon hits in CMU and CMP, whereas the CMX trigger only requires hits in the CMX. A corresponding XFT track is also required.

Lepton $\eta$ and $\phi$ also affect the trigger efficiency. For $Z H \rightarrow l^{+} l^{-} b \bar{b}, 60 \%$ of events where the $Z$ decays to electrons are triggered by CEM, and $50 \%$ of events where the $Z$ decays to muons are triggered by the CMUP and CMX triggers combined [31].

Since this is a search, it is critical to maximize acceptance for $Z H$ signal. To that effect, we use a lepton selection looser than the standard CDF selection [34] [35]. At least one of the two leptons must meet the tight lepton requirements, however the other can meet the looser requirements defined below in table 4.4 for electrons and Table 4.5 for muons. Together the two leptons must have an invariant mass between 76 and $106 \mathrm{GeV} / \mathrm{c}^{2}$.

A natural quesiton to ask is why $Z \rightarrow \tau^{+} \tau^{-}$are not considered. Decays to $\tau^{+} \tau^{-}$are complicated by the additional decay of the $\tau$ to hadrons or charged leptons and neutrinos. In cases where the $\tau$ secondarily decays to hadrons, the event cannot match the two $e$ or $\mu$ requirement. If the selection were modified to allow $\tau$ hadronic decays, large new QCD backgrounds would be introduced. The case where both $\tau$ decay leptonically to an opposite sign pair is very rare $(6 \%)$, and even then there would be four neutrinos present in the event, leading to high missing transverse energy, making it unsuitable for our selection.

\subsubsection{Jets}

Figure 2.16 tells us that a low mass Higgs boson decays to $b \bar{b}$ most of the time. QCD requires the $b$ quarks hadronize into jets, which are then measured in the calorimeter. Kinematics favor $b$ quarks with high transverse momentum because the Higgs is so massive compared to the $b$ quark. Figure 4.1 shows that the leading $b$ quark almost always has $p_{T}$ over $25 \mathrm{GeV} / \mathrm{c}$ and the second $b$ quark almost always has $p_{T}$ over 15 $\mathrm{GeV} / \mathrm{c}$. Setting the jet cuts at those values reduces a significant amount of background 


\begin{tabular}{c|c}
\hline Tight Electron Selection & Loose Electron Selection \\
\hline$E_{T}>18 \mathrm{GeV}$ & $E_{T}>10 \mathrm{GeV}$ (central) \\
$p_{T}>9 \mathrm{GeV} / \mathrm{c}$ & $E_{T}>18 \mathrm{GeV}$ (plug) \\
$\mathrm{Had} / \mathrm{Em}<0.055+0.0004 \cdot \mathrm{E}$ & $\mathrm{Had} / \mathrm{Em}<0.055+0.00045 \cdot \mathrm{E}$ \\
$E / P<2.5+0.015 E_{T}$ & Isolation $\cdot E_{T}^{\text {raw }} / E_{T}^{\mathrm{corr}}<0.1$ \\
$\left|Z_{\text {vertex }}\right|<60 \mathrm{~cm}$ & $p_{T}>5 \mathrm{GeV} / \mathrm{c}$ (if central) \\
$|\eta|<1$ & $\left|Z_{\text {vertex }}\right|<60 \mathrm{~cm}$ \\
$L_{\mathrm{shr}}<0.2$ & Fiducial \\
Isolation $\cdot E_{T}^{\text {raw }} / E_{T}^{\text {corr }}<0.1$ & \\
$-3.0<Q \cdot|\Delta x|<1.5$ & \\
$\chi_{\text {strip }}^{2}<25.0$ & \\
$\left|Z_{\text {electron }}-Z_{\text {vertex }}\right|<3 \mathrm{~cm}$ & \\
2 stereo and 2 axial super-layer segments & \\
\hline
\end{tabular}

Table 4.4: Electron requirements

\begin{tabular}{c|c|c}
\hline $\begin{array}{c}\text { Tight Muon Selection } \\
\left(p_{T}>20 \mathrm{GeV} / \mathrm{c}\right)\end{array}$ & $\begin{array}{c}\text { Loose Muon Selection } \\
\left(p_{T} \leq 20 \mathrm{GeV} / \mathrm{c}\right)\end{array}$ & $\begin{array}{c}\text { Loose Muon Selection } \\
\left(p_{T}>20 \mathrm{GeV} / \mathrm{c}\right)\end{array}$ \\
\hline Had Energy $<6 \mathrm{GeV}$ & Had Energy $<6 \mathrm{GeV}$ & Had Energy $<12 \mathrm{GeV}$ \\
Em Energy $<2 \mathrm{GeV}$ & Em Energy $<2 \mathrm{GeV}$ & Em Energy $<4 \mathrm{GeV}$ \\
Isolation $<0.1$ & Isolation $<0.1$ & Isolation $<0.1$ \\
$d_{0}<0.2(\mathrm{w} /$ o silicon hits) & $d_{0}<0.2(\mathrm{w} / \mathrm{o}$ silicon hits $)$ & \\
$d_{0}<0.02(\mathrm{w} /$ silicon hits) \\
$\geq 3$ stereo segments & $d_{0}<0.02(\mathrm{w} /$ silicon hits $)$ & \\
$\geq 3$ axial segments & $\geq 1$ COT segment & $\geq 1$ COT segment \\
\hline Tight CMUP requirements & & \\
$|\Delta x|_{C M U}<3.0 \mathrm{~cm}$ & & \\
$|\Delta x|_{C M P}<5.0 \mathrm{~cm}$ & & \\
\hline Tight CMX requirements & & \\
$|\Delta x|_{C M X}<6.0 \mathrm{~cm}$ & & \\
$\rho$ & & \\
\hline
\end{tabular}

Table 4.5: Muon requirements. Any of the three sets of criteria can be satisfied. Loose muons are not required to have muon detector stubs. $\rho$ is the COT exit radius.

while maintaining most of the signal.

We choose to use $\Delta R=0.4$ cone jets, meaning the distance in the $\eta, \phi$ plane $(\Delta R=$ $\left.\sqrt{\Delta \phi^{2}+\Delta \eta^{2}}\right)$ is 0.4 . This is used when finding the bounds of the jet in the reconstruction software. Larger cones allow capture of more of the partons that make up a jet at the expense of more noise. 


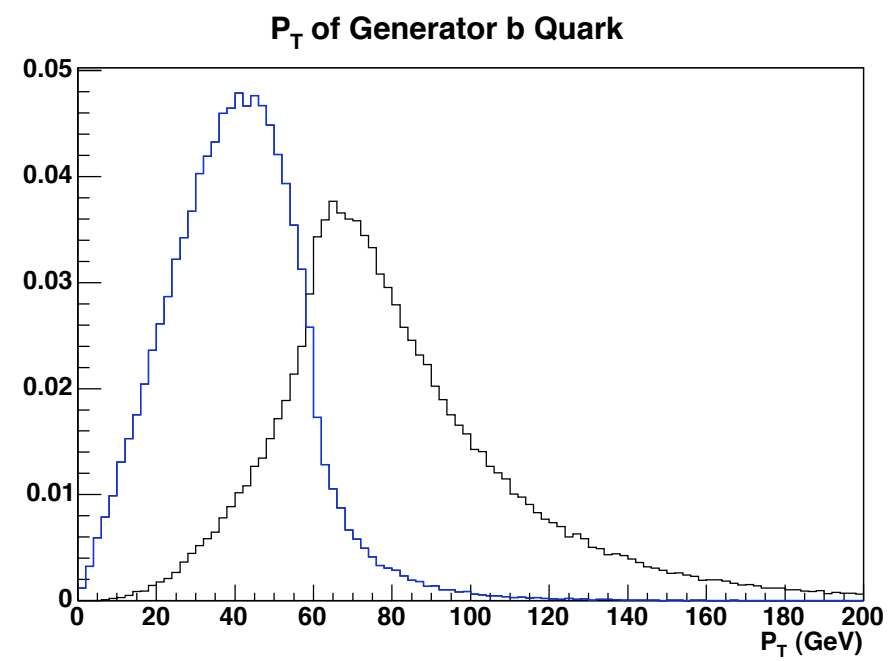

FIGURE 4.1: $Z H$ Higgs decay $b$-quark kinematics. Transverse momenta (top) and of the two $b$ quarks produced [31]

\subsection{2 b-Tagging}

Higgs events are sometimes hard to separate from Drell-Yan events based on kinematics alone. However, the Higgs boson strongly favors decay to $b \bar{b}$ over other hadronic decays. By searching for this $b$ quark signature, we greatly enhance sensitivity to signal. We use the SECVTX $b$-tagging algorithm [36], which uses the Silicon detector to look for a secondary vertex in the event since $b$ quarks have such a short lifetime.

We separate events into two categories, those with two 'loose' $b$-tags, and those with one or more $b$-tags, preferring to classify an event as two loose if both criteria are satisfied. The signal and background partition into the two categories differently, leading to a higher signal to background ratio in the two loose tag channel. This fact is later used to enhance sensitivity (see chapter 6).

$b$-tags are very good at reducing Drell-Yan + jets background. Requiring a $b$-tag cuts $\sim 98 \%$ of light flavor $(u, d, s$ quark) jet events and $\sim 80 \%$ of heavy flavor $(c, b)$ jet events. Fakes, which are similar to Drell-Yan + jets kinematically, are events where a lepton is reconstructed as a jet. Fakes achieve similar reduction factors. In contrast, $Z H$ is reduced 
by only $35 \%$ by the $b$-tag requirement, dramatically improving the signal to background ratio.

\subsection{Backgrounds}

The selection must be balanced between cutting out background while maintaining sufficient signal acceptance. This leads to three large backgrounds to be accounted for: Drell-Yan $(Z+$ jets), $t \bar{t}$ pair production, and diboson $(W Z / Z Z)$ production, shown in Figure 4.2 and Figure 4.3.

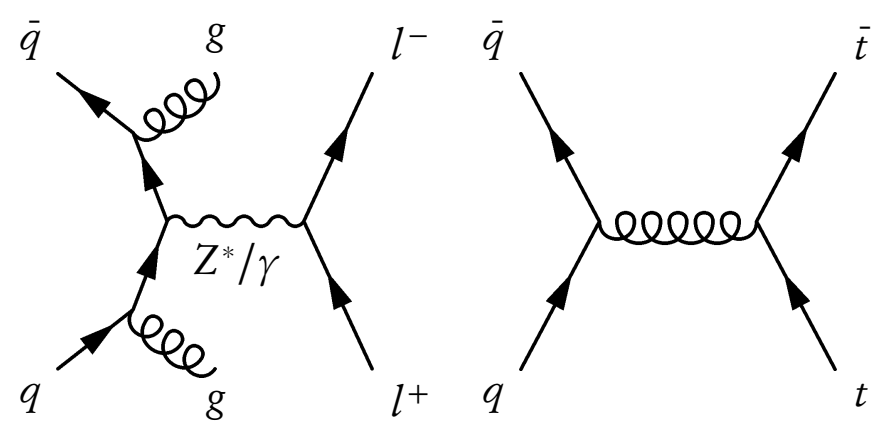

FIGURE 4.2: Tree level diagrams for the Drell-Yan + jets and $t \bar{t}$ background processes

The Drell-Yan background events typically have two jets that come from initial state radiation. This produces the requisite two jet, two lepton signature that passes our selection. The $t \bar{t}$ pair production has a $\sim 100 \%$ branching ratio to $W W b \bar{b}$. Both $W$ bosons can decay hadronically or leptonically. In the case where both decay leptonically, the final state is $l^{+} l^{-} \nu \bar{v} b \bar{b}$. Since the neutrinos are invisible to the detector, the observed state is the same as our selection, except with high missing transverse energy.

Diboson production results in $W Z / Z Z$. $W W$ is also produced, but is entirely eliminated by kinematic selection. $W Z$ also has very low acceptance for kinematic reasons, so we only expect 1.0 event. However $Z Z$ can have one $Z$ decay hadronically to $b \bar{b}$ and the other to $l^{+} l^{-}$. Kinematically, ZZ is very similar to $Z H$ with the exception of the reconstructed dijet mass distribution. This makes it difficult to separate from 


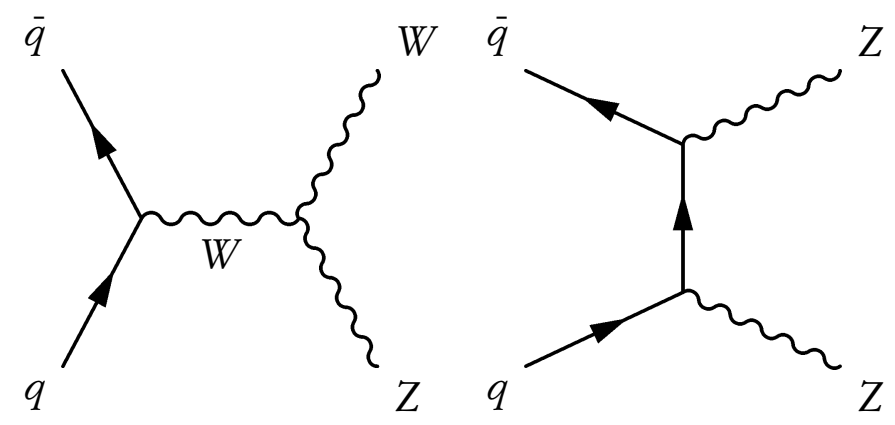

FIGURE 4.3: Tree level diagrams for the diboson $W W / W Z / Z Z$ processes

$Z H$ and consequently significant amounts of $Z Z$ are allowed into the selection. The $W W / W Z / Z Z$ category in Tables 4.9-4.8 is $92 \% Z Z$ and $8 \% W Z$ after tag requirements.

\subsection{Signal Acceptance}

Figure 2.14 gives $Z H$ production cross sections at the Tevatron, and Figure 2.16 gives Higgs boson branching ratios. The Feynman diagram for the production process is shown as Figure 4.4. With $2.7 \mathrm{fb}^{-1}$ of collected data, based on ALPGEN [37] Monte Carlo and the effects of trigger and selection efficiencies we expect events as shown in table 4.6.

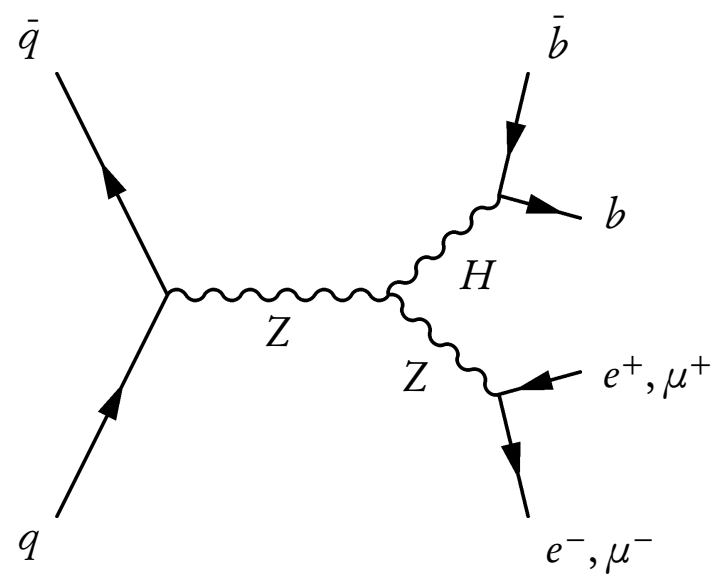

FIGURE 4.4: $\mathrm{ZH} \rightarrow l^{+} l^{-} b \bar{b}$ production mechanism 


\begin{tabular}{c|cc|c|cc}
\hline$M_{H}$ & $\sigma(\mathrm{fb})$ & $B R(H \rightarrow b \bar{b})$ & Pretag & 1 Tight & 2 Loose \\
\hline 100 & 169.0 & 0.803 & 4.52 & 1.97 & 0.96 \\
105 & 145.0 & 0.785 & 4.12 & 1.81 & 0.93 \\
110 & 125.0 & 0.758 & 3.23 & 1.41 & 0.71 \\
115 & 107.9 & 0.716 & 2.88 & 1.25 & 0.65 \\
120 & 93.7 & 0.659 & 2.60 & 1.13 & 0.58 \\
125 & 81.6 & 0.587 & 1.79 & 0.79 & 0.42 \\
130 & 71.2 & 0.502 & 1.31 & 0.57 & 0.31 \\
135 & 62.4 & 0.410 & 0.93 & 0.45 & 0.25 \\
140 & 54.8 & 0.319 & 0.68 & 0.31 & 0.17 \\
145 & 48.2 & 0.235 & 0.44 & 0.21 & 0.12 \\
150 & 37.6 & 0.160 & 0.28 & 0.12 & 0.07 \\
\hline
\end{tabular}

Table 4.6: Expected number of $Z H \rightarrow l^{+} l^{-} b \bar{b}$ events for Higgs masses between 100 and $150 \mathrm{GeV} / \mathrm{c}^{2}$ in the two loose $b$-tag and one tight $b$-tag channels. Pretag indicates all selection requirements except $b$-tagging.

\subsection{Event Totals}

\subsubsection{Pretag}

\begin{tabular}{lrrr} 
Source & $e e$ & $\mathrm{~mm}$ & $l l$ \\
\hline$Z \rightarrow e e$ & $3768.54 \pm 986.85$ & $0.00 \pm 0.00$ & $3768.54 \pm 986.85$ \\
$Z \rightarrow e e+$ hf & $391.71 \pm 68.15$ & $0.00 \pm 0.00$ & $391.71 \pm 68.15$ \\
$Z \rightarrow \mu \mu$ & $0.04 \pm 0.01$ & $2685.53 \pm 681.26$ & $2685.57 \pm 681.26$ \\
$Z \rightarrow \mu \mu+$ hf & $0.00 \pm 0.00$ & $263.07 \pm 45.68$ & $263.07 \pm 45.68$ \\
$Z \rightarrow \tau \tau$ & $3.07 \pm 0.63$ & $0.66 \pm 0.13$ & $3.73 \pm 0.65$ \\
$Z \rightarrow \tau \tau+$ hf & $0.15 \pm 0.04$ & $0.01 \pm 0.00$ & $0.16 \pm 0.04$ \\
$W W, W Z, Z Z$ & $91.76 \pm 12.73$ & $65.87 \pm 9.16$ & $157.62 \pm 15.69$ \\
Fakes & $640.28 \pm 111.30$ & $35.80 \pm 13.61$ & $676.08 \pm 112.12$ \\
$t t$ & $19.13 \pm 3.83$ & $14.86 \pm 2.97$ & $33.99 \pm 4.85$ \\
\hline \hline Total & $4916.33 \pm 995.44$ & $3067.02 \pm 683.00$ & $7986.35 \pm 1207.22$ \\
Data & 4297 & 2960 & 7257
\end{tabular}

Table 4.7: Pretag background expectation 

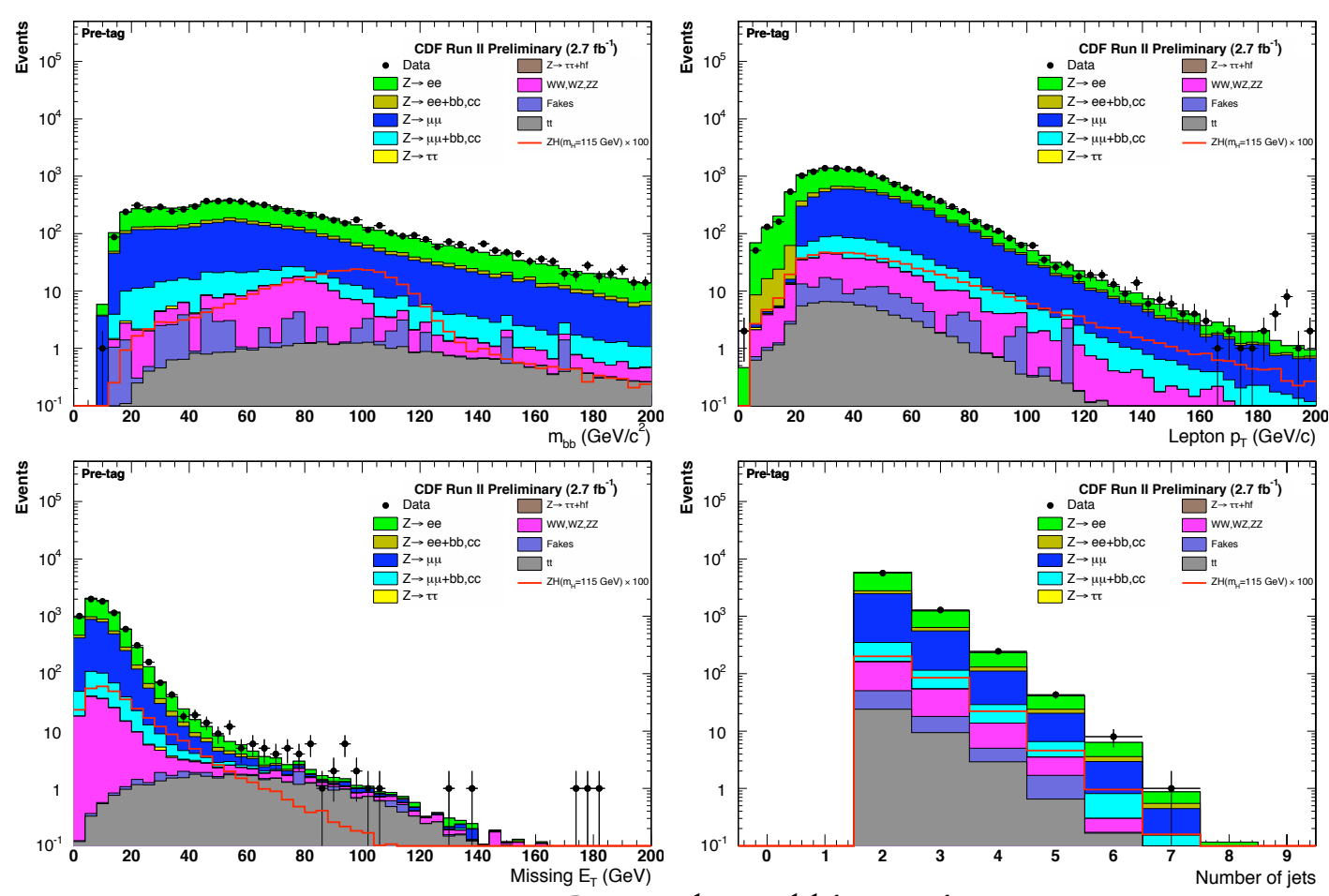

FIGURE 4.5: Pretag channel kinematics

\subsubsection{Double Loose b-Tag}

\begin{tabular}{lrrr} 
Source & $e e$ & $\mu \mu$ & $l l$ \\
\hline$Z \rightarrow e e$ & $2.93 \pm 0.73$ & $0.00 \pm 0.00$ & $2.93 \pm 0.73$ \\
$Z \rightarrow e e+$ hf & $11.39 \pm 2.76$ & $0.00 \pm 0.00$ & $11.39 \pm 2.76$ \\
$Z \rightarrow \mu \mu$ & $0.00 \pm 0.00$ & $2.55 \pm 0.61$ & $2.55 \pm 0.61$ \\
$Z \rightarrow \mu \mu+$ hf & $0.00 \pm 0.00$ & $8.12 \pm 1.93$ & $8.12 \pm 1.93$ \\
$Z \rightarrow \tau \tau$ & $0.00 \pm 0.00$ & $0.00 \pm 0.00$ & $0.00 \pm 0.00$ \\
$Z \rightarrow \tau \tau+$ hf & $0.00 \pm 0.00$ & $0.00 \pm 0.00$ & $0.00 \pm 0.00$ \\
$W Z, Z Z$ & $1.69 \pm 0.33$ & $1.24 \pm 0.24$ & $2.94 \pm 0.41$ \\
Fakes & $0.03 \pm 0.01$ & $0.02 \pm 0.01$ & $0.04 \pm 0.01$ \\
$t \bar{t}$ & $4.38 \pm 0.88$ & $3.29 \pm 0.66$ & $7.66 \pm 1.09$ \\
\hline \hline Total & $20.42 \pm 3.01$ & $15.21 \pm 2.14$ & $35.63 \pm 3.69$ \\
Data & 16 & 16 & 32
\end{tabular}

Table 4.8: Double $b$-tag background expectation 

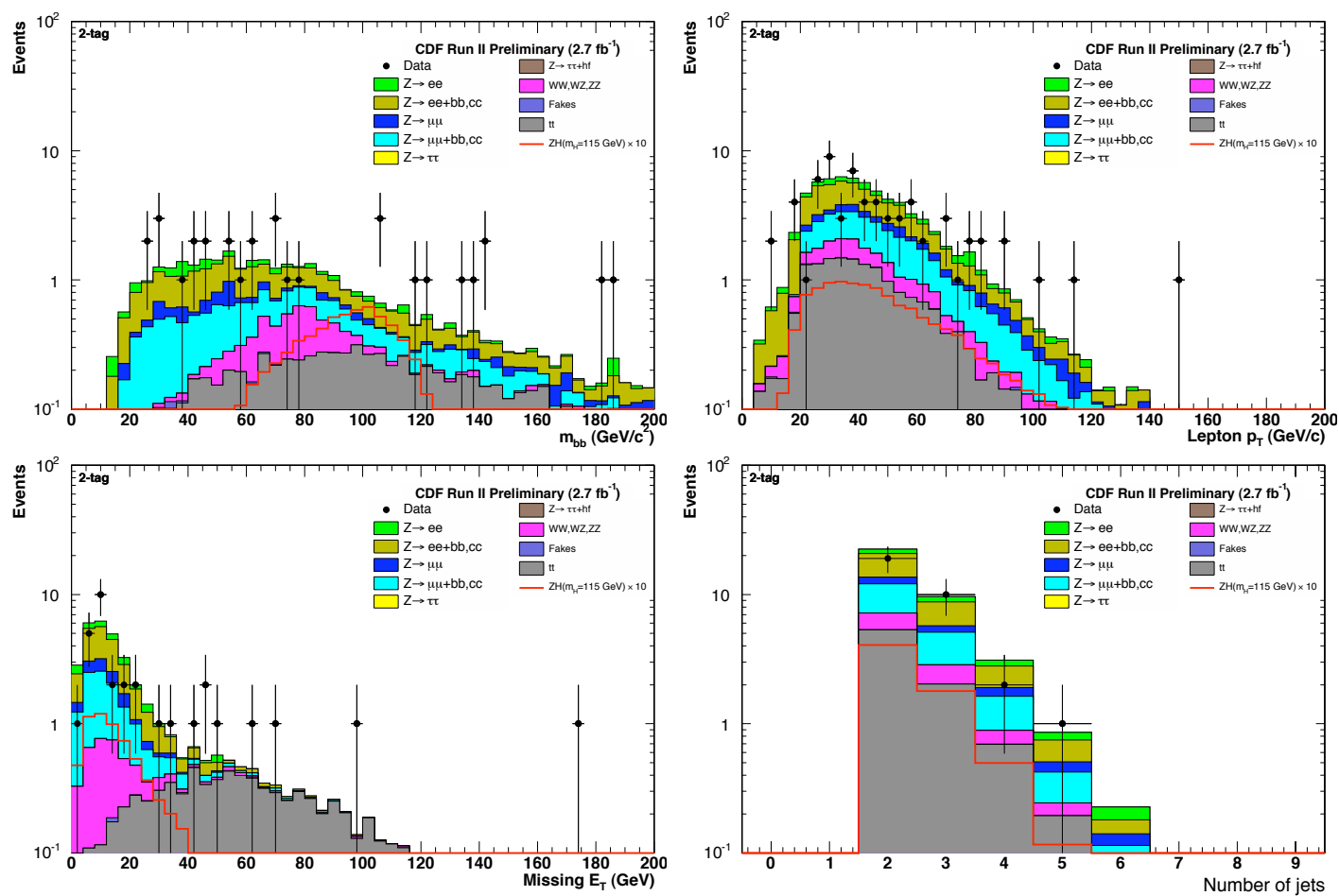

FIGURE 4.6: Double $b$-tag channel kinematics

\subsubsection{Single Tight b-tag}

\begin{tabular}{lrrr} 
Source & $e e$ & $\mu \mu$ & $l l$ \\
\hline$Z \rightarrow e e$ & $70.67 \pm 18.84$ & $0.00 \pm 0.00$ & $70.67 \pm 18.84$ \\
$Z \rightarrow e e+$ hf & $63.03 \pm 11.43$ & $0.00 \pm 0.00$ & $63.03 \pm 11.43$ \\
$Z \rightarrow \mu \mu$ & $0.00 \pm 0.00$ & $58.89 \pm 14.89$ & $58.89 \pm 14.89$ \\
$Z \rightarrow \mu \mu+$ hf & $0.00 \pm 0.00$ & $44.21 \pm 8.14$ & $44.21 \pm 8.14$ \\
$Z \rightarrow \tau \tau$ & $0.05 \pm 0.02$ & $0.02 \pm 0.01$ & $0.07 \pm 0.02$ \\
$Z \rightarrow \tau \tau+$ hf & $0.04 \pm 0.01$ & $0.01 \pm 0.00$ & $0.04 \pm 0.01$ \\
$W Z, Z Z$ & $6.64 \pm 1.04$ & $4.97 \pm 0.79$ & $11.61 \pm 1.30$ \\
Fakes & $12.80 \pm 6.40$ & $3.09 \pm 1.55$ & $15.89 \pm 6.58$ \\
$t \bar{t}$ & $7.71 \pm 1.54$ & $6.15 \pm 1.23$ & $13.86 \pm 1.97$ \\
\hline \hline Total & $160.94 \pm 23.15$ & $117.34 \pm 17.10$ & $278.28 \pm 28.78$ \\
Data & 152 & 106 & 258
\end{tabular}

Table 4.9: Single $b$-tag background expectation 

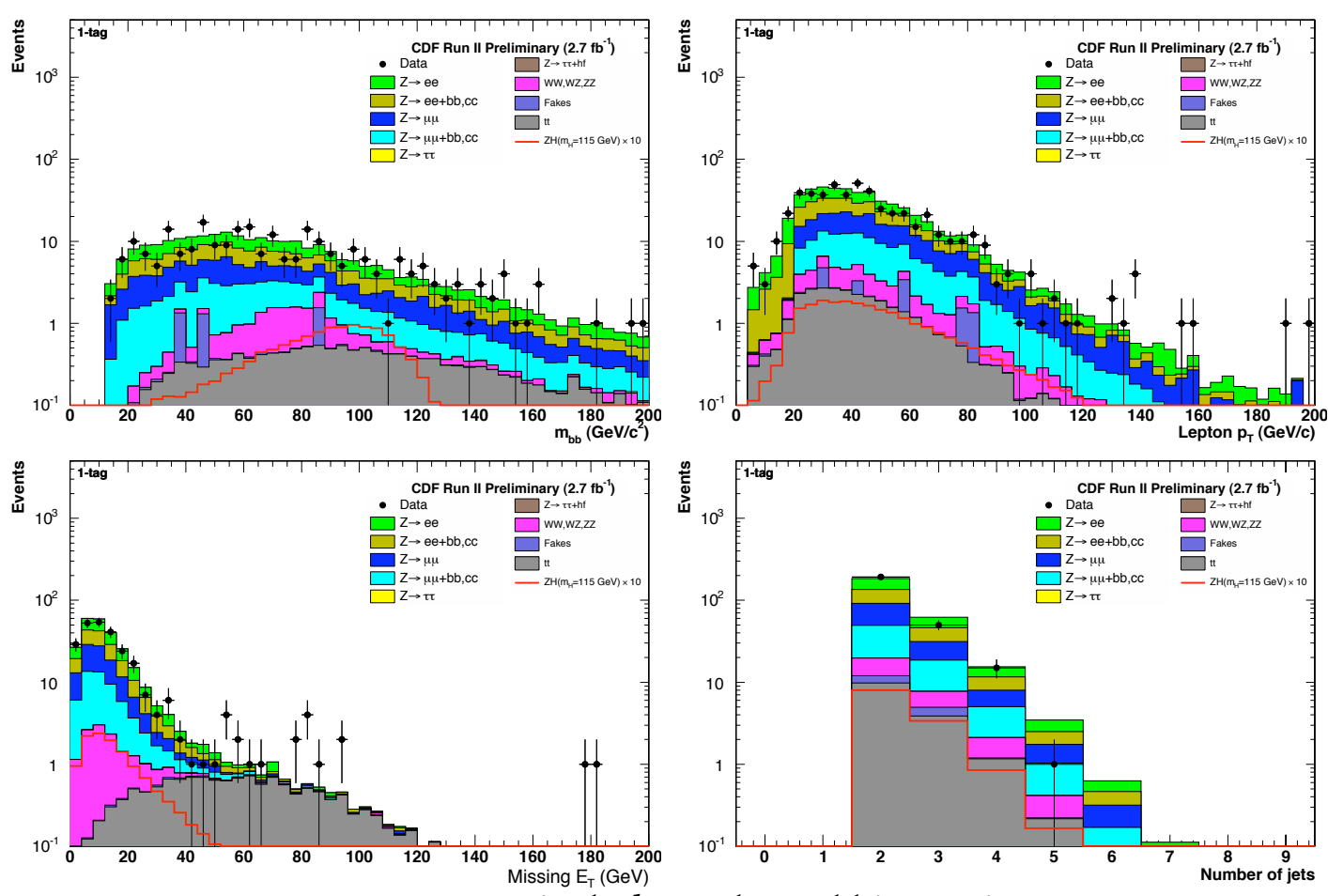

FIGURE 4.7: Single $b$-tag channel kinematics 


\section{5}

\section{Matrix Elements}

Perturbation theory allows calculation of transition probabilities between quantum states. Since the standard model is a perturbative theory, in an ideal world, the probability of a transition between the initial state $p \bar{p}$ and the final measured partons would be calculable. In the real world, many specifics about the initial and final states are unknowable. But, by integrating over unknown quantities and making a few assumptions, an analogous probability can be obtained. If we call the complete set of measured event kinematics $\mathbf{x}$, and the set of physics parameters (particle masses, SM couplings, etc.) used to perform the calculation $\Theta$, the probability density function $P_{j}$ of the $j^{\text {th }}$ process is proportional to the differential cross section in equation 5.1.

$$
P_{j}(\mathbf{x} \mid \boldsymbol{\Theta})=\frac{1}{\sigma(\boldsymbol{\Theta})} \frac{d \sigma(\boldsymbol{\Theta})}{d \mathbf{x}}
$$

\subsection{Matrix Element Approximations}

Without any approximations, if the initial and final states could be known exactly (including quantum numbers that are unknowable like color), the differential cross section would simply be the integrated matrix element transition probability calculated using 
perturbation theory. Since there is a great deal we do not know about the initial and final states of the we must make the following approximations

\subsubsection{Initial State}

Since protons and anti-protons are composite particles, the actual interaction occurs between the constituents of the protons, the quarks and gluons. Unfortunately we do not know the exact kinematics of the quarks and gluons. We also do not know color and spin configurations of the colliding particles. We do not even know if the interacting particles were quarks or if they were gluons; we only know that we collided $p$ with $\bar{p}$.

To account for this broad lack of knowledge of the initial state, we use a parton density function (PDF) from the CTEQ group [38], version 6.1, which is a parametrization

of the momenta of the components of a proton. We integrate over the parton density functions for both the $p$ and $\bar{p}$, leading to

$$
\frac{d \sigma(\boldsymbol{\Theta})}{d p_{f}}=\int d \Phi\left|\mathscr{M}_{j}\left(p_{i}, p_{f} \mid \boldsymbol{\Theta}\right)\right|^{2} f_{\mathrm{PDF}}\left(p_{i}, p_{1}\right) \bar{f}_{\mathrm{PDF}}\left(p_{i}, p_{2}\right)
$$

where $f_{\mathrm{PDF}}$ is the parton density function for the proton and $\bar{f}_{\mathrm{PDF}}$ is the parton density function for the anti-proton. Because the PDF returns a probability of measuring a momenta (given some collider and theory parameters), it allows integration over all accessible regions of the 4-momentum for initial state particles. Usually initial state particles are not virtual, which reduces the dimension of integration by one.

\subsubsection{Final State}

QCD confinement makes it impossible to measure color, spin, or momentum of the actual final state quarks. The quarks hadronize into jets which loosely resemble the original quark. In the case of backgrounds, there can be jets from gluons which hadronize as well. Additionally all of the final state partons' measured variables are affected by limited detector resolution and granularity. 
To account for this we introduce the transfer functions $W\left(p_{f}, \mathbf{x}\right)$. Each transfer function links a final state parton to the corresponding measured reconstructed object. Since there are a range of parton momenta that can result in the measured value, this implies an integration over final parton momenta. Changing variables for the differential cross section and including the additional integrations in $d \Phi$, equation 5.2 becomes

$$
\frac{d \sigma(\boldsymbol{\Theta})}{d \mathbf{x}}=\int d \Phi\left|\mathscr{M}_{j}\left(p_{i}, p_{f} \mid \boldsymbol{\Theta}\right)\right|^{2}\left[\prod W\left(p_{f}, \mathbf{x}\right)\right] f_{\mathrm{PDF}}\left(p_{i}, p_{1}\right) \bar{f}_{\mathrm{PDF}}\left(p_{i}, p_{2}\right)
$$

We make a few simplifying assumptions about the process and detector to reduce the necessary integrations.

- Leptons are measured perfectly. This is equivalent to the lepton transfer function being a three dimensional delta function.

- Jet angles are measured perfectly, and jet energies are parametric functions of parton energy. The jet transfer functions can be expressed as

$$
\delta\left(\theta_{\text {jet }}-\theta_{\text {parton }}\right) \delta\left(\phi_{\text {jet }}-\phi_{\text {parton }}\right) W\left(E_{\text {parton }}, E_{\text {jet }}\right)
$$

where $W\left(E_{p}, E_{j}\right)$ is the parametrized relationship between parton energy and jet energy.

- Incoming partons are massless (good approximation for light quarks), and have no transverse momentum.

- The two leading jets are $b$ quark jets.

- Final state leptons are massless, and final state $b$ quarks have an invariant mass of $4.7 \mathrm{GeV} / \mathrm{c}^{2}$.

Additionally, each production process has multiple Feynman diagrams, which requires

$$
\left|\mathscr{M}_{j}\left(p_{i}, p_{f} \mid \boldsymbol{\Theta}\right)\right|^{2} \rightarrow \sum_{a}\left|\mathscr{M}_{j}^{(a)}\left(p_{i}, p_{f} \mid \boldsymbol{\Theta}\right)\right|^{2}
$$


where the index $a$ runs over the different Feynman diagrams available to a production process. At minimum, the number of incoming quark flavors and colors must be summed over. This makes the general form of the differential cross section

$$
\frac{d \sigma(\boldsymbol{\Theta})}{d \mathbf{x}}=\int d \Phi\left[\sum_{a}\left|\mathscr{M}_{j}^{(a)}\left(p_{i}, p_{f} \mid \boldsymbol{\Theta}\right)\right|^{2}\right]\left[\prod W\left(p_{f}, \mathbf{x}\right)\right] f_{\mathrm{PDF}}\left(p_{i}, p_{1}\right) \bar{f}_{\mathrm{PDF}}\left(p_{i}, p_{2}\right)
$$

\subsection{Jet Transfer Function}

As mentioned in the simplifications, the $W\left(E_{\text {parton }}, E_{\text {jet }}\right)$ function links the measured jet

energy to a possible parton energy $E_{\text {parton }}$ with some probability. By integrating over all parton energies, this allows removal of the unknown parton energy from the differential cross section.

To get this transfer function we start with a Monte Carlo sample of $\mathrm{ZH} \rightarrow l^{+} l^{-} b \bar{b}$ events. We eliminate jets in the final state that can be traced to initial state radiation (ISR) quarks or gluons. For $b$ quark jets (matched to partons within a $\Delta R=0.4$ cone), we find the parton energy $E_{\text {parton }}$ and the Level 5 corrected ([32]) jet energy $E_{\text {jet }}$. We then perform a fit of $\delta=E_{p}-E_{j}$ with our functional form, which we take to be a double Gaussian, defined as equation 5.5 [39].

$$
W_{j}(\delta)=\frac{1}{\sqrt{2 \pi}\left(p_{2}+p_{3} p_{5}\right)}\left[\exp \left(\frac{-\left(\delta-p_{1}\right)^{2}}{2 p_{2}^{2}}\right)+p_{3} \exp \left(\frac{-\left(\delta-p_{4}\right)^{2}}{2 p_{5}^{2}}\right)\right]
$$

where each $p_{i}$ depends linearly on $E_{p}$ :

$$
p_{i}=a_{i}+b_{i} E_{p}
$$

The fitted transfer function, has constants given in Table 5.1 and Figure 5.1-5.2. 


\begin{tabular}{ccc}
\hline$p_{i}$ & $a_{i}$ & $b_{i}$ \\
\hline$p_{1}$ & $-3.99 \pm 0.15$ & $-0.035 \pm 0.002$ \\
$p_{2}$ & $2.66 \pm 0.13$ & $0.072 \pm 0.001$ \\
$p_{3}$ & $0.652 \pm 0.020$ & $0.00 \pm 0.000$ \\
$p_{4}$ & $0.374 \pm 0.23$ & $-0.274 \pm 0.005$ \\
$p_{5}$ & $7.82 \pm 0.20$ & $0.092 \pm 0.003$ \\
\hline
\end{tabular}

Table 5.1: Constants for the $W\left(E_{\text {parton }}, E_{\text {jet }}\right)$ transfer function.

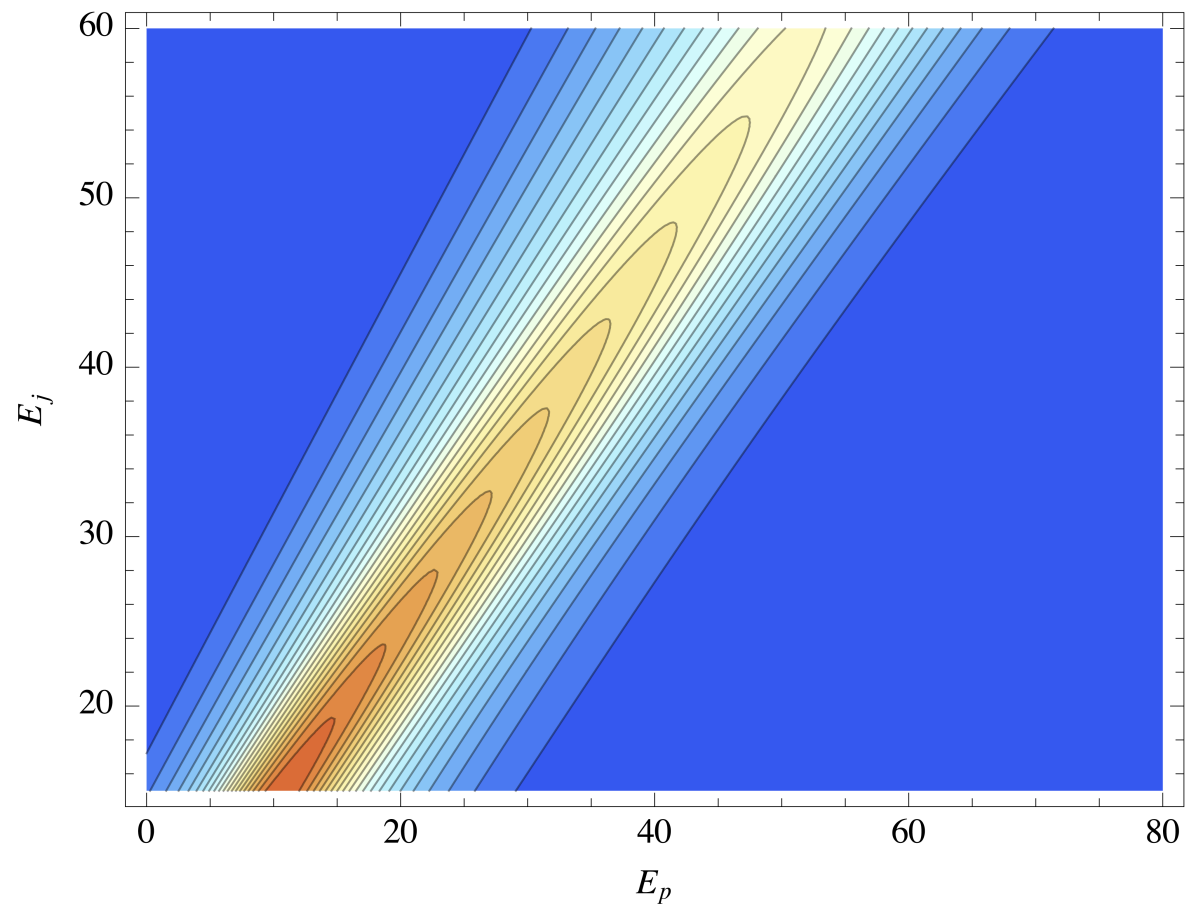

FIGURE 5.1: The parton-jet transfer function contours are shown. As expected, at low transverse energies, the jet and parton energies match to within an offset and smearing. The smearing increases as $E_{j}$ and $E_{p}$ increase. 

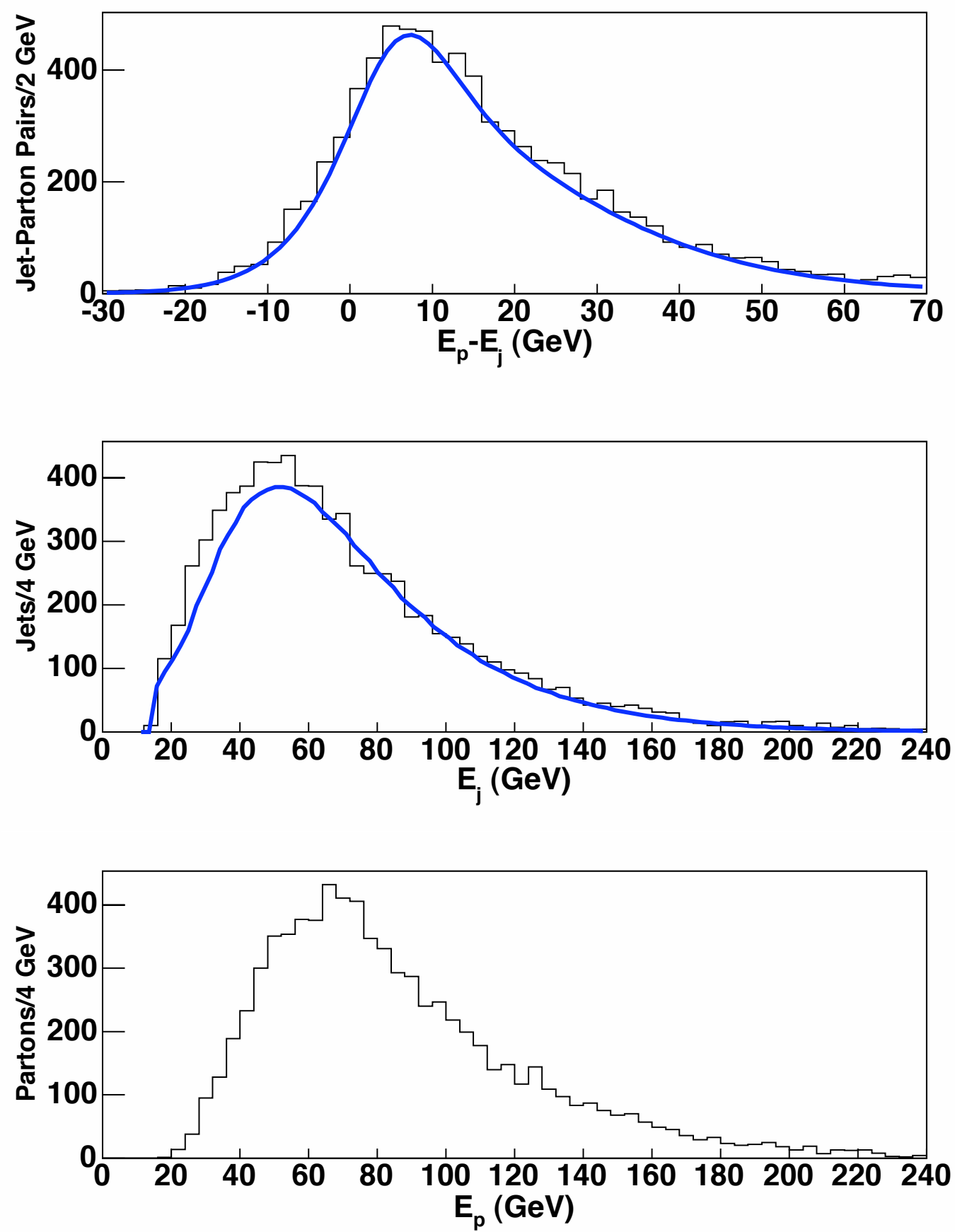

FIGURE 5.2: Fitted parton and jet spectra. The first plot shows $E_{p}-E_{j}$, which is the fitted distribution. The second shows the $E_{j}$ distribution given the $E_{p}$ distribution as the input distribution in the third plot. All plots are from Monte Carlo with $M_{H}=120$ GeV $/ c^{2}$ 


\subsection{Processes}

All four matrix elements we use for the $Z H$ search integrate over the following variables:

- Lepton momenta (x2)

- Initial parton momenta (x2)

- Final state quark momenta (x2)

- $\mathrm{x}$ and $\mathrm{y}$ components of system momenta (recoil)

To perform the integration, we use the Monte Carlo importance sampling technique VEGAS from the GNU Scientific Library (GSL) version 1.9. Although a full description of VEGAS is best left to [40], the key feature is a multi-pass iterative buildup of a sampling distribution for the integrand. Moreover, the sampling distribution is adaptive to the integrand, being determined to higher precision in regions of higher contribution to the integral. Since the sampling distribution closely follows the integrand, the variance of the points chosen by VEGAS is lower than the variance of points from a more naive approach.

A more in depth explanation of the matrix elements is presented in [39], including the variables of integration, changes of variables required and the corresponding Jacobians.

\subsection{1 $\mathrm{ZH} \rightarrow l^{+} l^{-} b \bar{b}$ and $\mathrm{ZZ}$}

Here we use Monte Carlo for FeMtobarn processes (MCFM) [41, 42] to calculate

$$
\sum_{a}\left|\mathscr{M}_{j}^{(a)}\left(p_{i}, p_{f} \mid \boldsymbol{\Theta}\right)\right|^{2}
$$

In addition to the integration needed for all matrix elements, there is an additional integration over the unknown intermediate $Z$ and $H$ bosons (see Figure 2.17). We use the 
known $Z$ boson mass and width and the theoretical $H$ pole mass parameters to reduce the dimensionality of the integration.

The $Z Z \rightarrow l^{+} l^{-} b \bar{b}$ matrix element only differs from the $Z H \rightarrow l^{+} l^{-} b \bar{b}$ in terms of the kinematic distributions of the hadronic decay. The dijet mass for $Z Z$ events will reconstruct to the $Z$ boson pole mass instead of the Higgs boson pole mass.

\subsubsection{Z $\mathrm{Z}$ jets}

We use MadEvent/MadGraph [43] to calculate $|\mathscr{M}|^{2}$ in the case of $Z+$ jets. Unlike the other processes, there are many different mechanisms of $Z+$ jets event production. At least 65 distinct Feynman diagrams result in a final state of two leptons and two jets.

In order to carry out the integration over all the 8 specified variables for a given event, a great many evaluations $(\mathscr{O}(100,000))$ of $|\mathscr{M}|^{2}$ are required. Although each diagram can be evaluated fairly quickly (milliseconds), in order to achieve higher speed, MadGraph does statistical sampling of possible diagrams, choosing to weight dominant processes more heavily (several orders of magnitude faster). Unlike other processes we have matrix elements for, there is no integration over intermediate particles in the event.

\subsection{3 $t \bar{t}$}

We expect two neutrinos in $t \bar{t}$ events. Since they are invisible and have three degrees of freedom in the massless approximation, together they introduce six degrees of freedom that must be integrated over. The momenta of the intermediate particles, $t \bar{t}, W^{ \pm}$must also be integrated over.

The $|\mathscr{M}|^{2}$ for $t \bar{t}$ can be analytically derived, as in $[44,45]$, reproduced here

$$
|\mathscr{M}|^{2}=\frac{g_{s}^{4}}{9} F \bar{F}\left(\left(2-\beta^{2} s_{q t}^{2}\right)-X_{s c}\right)
$$

where $\beta$ is the top quark velocity in the $q \bar{q}$ rest frame, $X_{s c}$ contains terms describing spin correlations between top quarks, $g_{s}$ is the strong coupling constant, $s_{q t}$ is the sine of the 
angle between the incoming parton and the top quark, and $F(\bar{F})$ are the top (anti-top) quark propagators respectively. 


\section{Analysis Technique}

The sensitivity of a search can be estimated from the empirical measure of

$$
\text { signal } / \sqrt{\text { background }}
$$

The combination of SM prediction and detector effects like selection and tagging produces a $S / \sqrt{B} \sim \mathscr{O}\left(10^{-1}\right)$. This is insufficient to do any kind of pure counting experiment, so advanced analysis techniques are required. Likelihood fitting allows extraction of the maximum amount of information from each event. We use the matrix elements as described in chapter 5 to form a likelihood function.

\subsection{Likelihood Fitting -1}

Likelihood functions in a parameter need not be unique [46]. We use the matrix elements described in the previous chapter to form a likelihood function for each event. Each event has a signal and background probability based on matrix elements, $P_{s}$ and a $P_{b}$ respectively. The expression

$$
L(S, \mathbf{x} \mid \boldsymbol{\Theta})=S P_{S}(\mathbf{x} \mid \boldsymbol{\Theta})+(1-S) P_{b}(\mathbf{x} \mid \boldsymbol{\Theta})
$$


is a per event likelihood in $S$ given event kinematics $\mathbf{x}$ and theory parameters $\boldsymbol{\Theta}$. Notice that this function is linear in the $S$ parameter, with the slope of the line indicating if the event is more signal-like or background-like. This definition of $\mathscr{L}$ possesses the normalization condition $\int \mathscr{L}=1$ given $\int P_{s} d \mathbf{x}=\int P_{b} d \mathbf{x}=1$, allowing $\mathscr{L}$ to be interpreted as the probability density function of the data.

The standard prescription for a likelihood fit on a set of data events is to take the product of the individual likelihood curves. Therefore equation 6.1 becomes

$$
\mathscr{L}(S)=\prod_{i} L\left(S, \mathbf{x}_{i}\right)=\prod_{i} S\left[P_{s}\left(\mathbf{x}_{i} \mid \boldsymbol{\Theta}\right)+(1-S) P_{b}\left(\mathbf{x}_{i} \mid \boldsymbol{\Theta}\right)\right]
$$

where $i$ is the index over events in the sample. As a matter of computability, we choose to instead maximize the log-likelihood

$$
\log \mathscr{L}(S)=\sum_{i} \log L\left(S, \mathbf{x}_{i}\right)=\sum_{i}\left[\log \left(S P_{s}\left(\mathbf{x}_{i} \mid \boldsymbol{\Theta}\right)+(1-S) P_{b}\left(\mathbf{x}_{i} \mid \boldsymbol{\Theta}\right)\right)\right]
$$

In the combined likelihood $S$ means signal fraction, $N_{s} / N$ for the sample under consideration. One of crucial properties of likelihood fitting is that the maximum likelihood estimator is normally distributed $[6,46]$.

\subsection{Resampling}

When considering events to fit, there are two complications. First, due to the slow speed of Monte Carlo generation and matrix element calculation, the statistics of events are limited. Secondly, the events that are generated by the slow Monte Carlo have weights based on factors like trigger efficiencies.

Resampling techniques allow the extraction of more information from a limited set of events. The technique we use is called bootstrapping, which involves taking random subsamples from the large pool of events. A cursory overview of bootstrapping can be found in [47] and detailed information in [48]. Barlow [49] states the estimation of an 
error allows $(\mathscr{N} / n)^{2}$ subsamples in the bootstrap, where $\mathscr{N}$ is the number of events in the pool, and $n$ is the number of events in the subsample. Since we are estimating the spread of a likelihood fit, the Barlow limit applies.

Bootstrapping allows us to form subsets of events similar to what we would expect from the $\mathrm{CDF}$ detector based on theory predictions. The theory predictions are given by Table 4.9 and Table 4.8. The subsets of events assembled are called psendo-experiments (PEs). Because the events are sampled from a large pool of events, it allows weighted sampling, as more heavily weighted events are part of the subsamples more often.

\subsection{Normalization}

To ensure normalization of the matrix elements described in chapter 5 , we perform an ad-hoc two component normalization using the likelihood fitter. Correctly normalized density functions are required for any likelihood fitting technique.

The four matrix elements, $Z H, Z J J, t \bar{t}$, and $Z Z$ are indexed by $j$. In principle, each should integrate to unity over the allowable phase space.

$$
\int d \mathbf{x} P_{j}(\mathbf{x} \mid \boldsymbol{\Theta})=\frac{1}{\sigma(\boldsymbol{\Theta})} \int d \mathbf{x} \frac{d \sigma(\boldsymbol{\Theta})}{d \mathbf{x}}=1
$$

Practically, we obtain this instead because the matrix elements were all coded independently by different people without paying attention to absolute cross section, only the shape of the probability density function.

$$
\int d \mathbf{x} P_{j}(\mathbf{x} \mid \boldsymbol{\Theta})=\int d \mathbf{x} \frac{d \sigma(\boldsymbol{\Theta})}{d \mathbf{x}}=\sigma(\boldsymbol{\Theta})
$$

In the likelihood fit, $P_{s}$ and $P_{b}$ must have a correct relative normalization. Since there are four matrix elements, and relative normalizations must commute, that leaves three independent normalization constants to find. We find the three normalization constants by choosing one matrix element as a basis and calibrating the other three using a two 
component likelihood fit. We choose to normalize the other matrix elements against the $t \bar{t}$ matrix element with a subsample size of 500 events. We choose 500 event subsamples because we wanted sufficient Monte Carlo statistics in the smallest sample to perform a sufficient number of resamples in the Barlow limit. We choose the $t \bar{t}$ process as the reference point to normalize other matrix elements against because it is strongly distinguishable from each of the others which enhances the statistical power of the fit. To normalize, we let $P_{s} \rightarrow P_{t \bar{t}}$ and $P_{b} \rightarrow P_{\{Z H, Z J J, Z Z\}}$. Then we run the likelihood

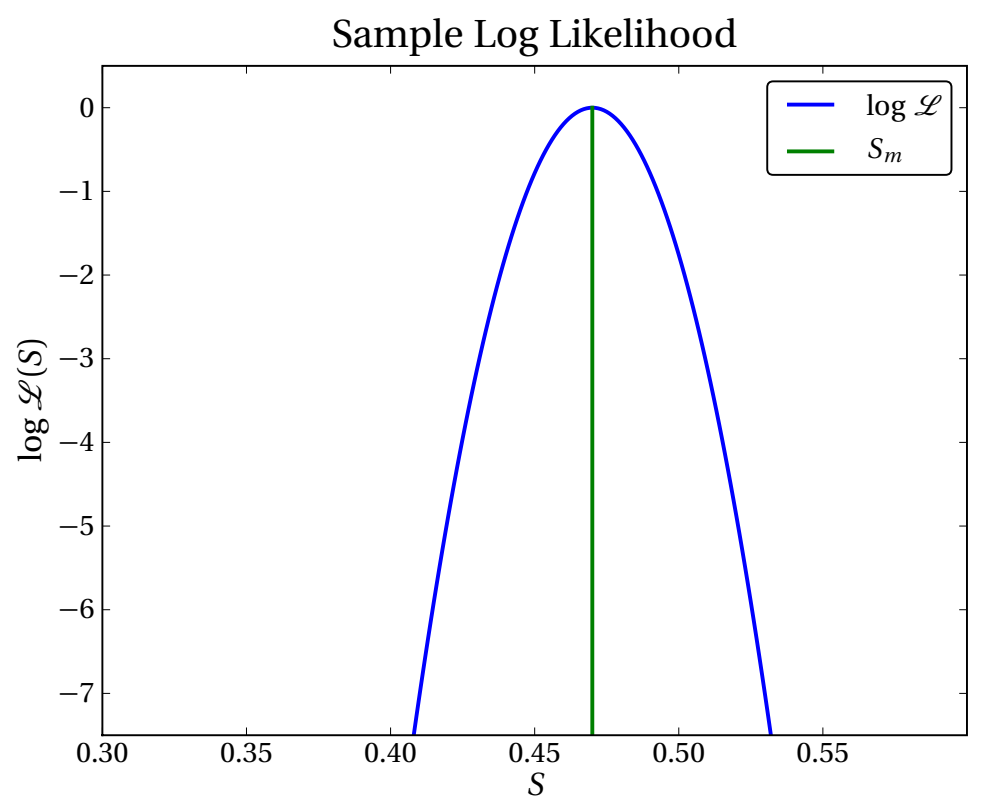

FIGURE 6.1: A single log likelihood curve, with the most likely value of $S$ marked as $S_{m}$

fitter on sets of events that are $50 \% t \bar{t}$ and $50 \%$ of $\{Z H, Z J J, Z Z\}$. One sample log likelihood curve is shown as Figure 6.1. The central limit theorem causes the polynomial expression for likelihood to become normally distributed, or correspondingly parabolic in log-likelihood space.

The bootstrap resampling technique introduces significant variation between subsamples. Each subsample produces a curve like the one in Figure 6.1, and each curve has a most likely value, $S_{m}$ (short for $S_{\text {measured }}$ ). Individual $S_{m}$ values are histogrammed for each 

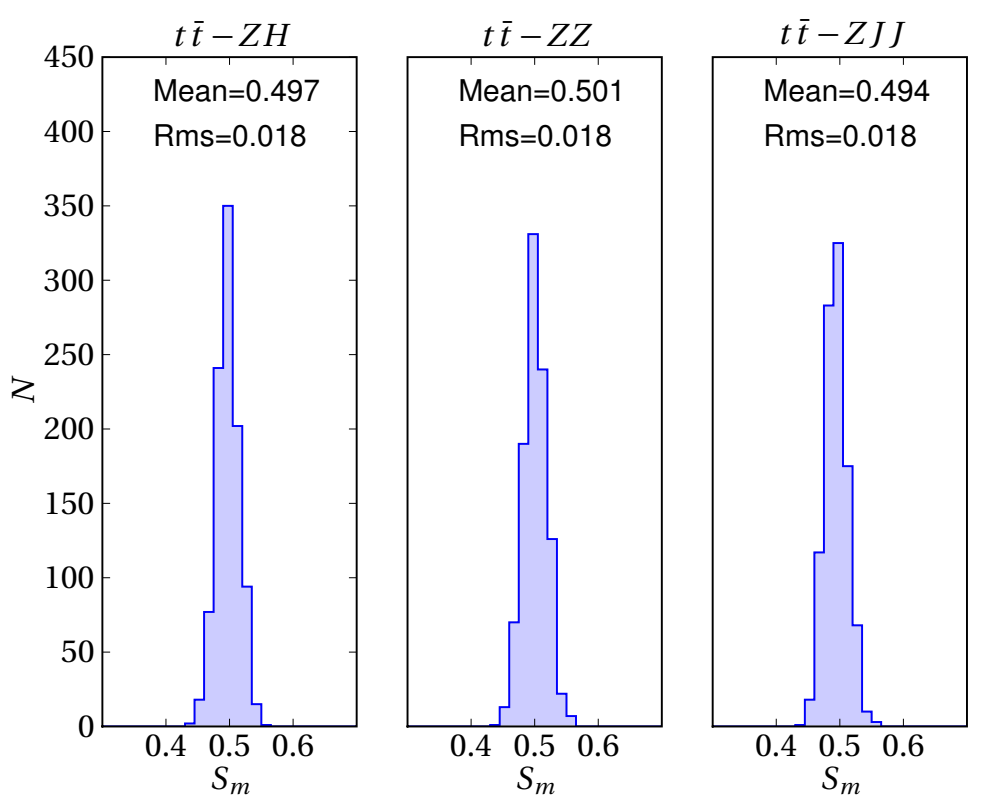

FIGURE 6.2: The aggregate histogram of most likely values $\left(S_{m}\right)$ for normalization sets of events at $m_{H}=115 \mathrm{GeV} / \mathrm{c}^{2}$. Each of the matrix elements were multiplied by the appropriate constant (Table 6.1) such that the histogram is centered at 0.5

of the three cases $Z H, Z J J, Z Z$, and the $P_{b}$ values are multiplied by normalization constants which are adjusted until the mean of the histogram occurs at 0.5 . The histograms for background and signal at $m_{H}=115 \mathrm{GeV} / \mathrm{c}^{2}$ are in Figure 6.2. Other Higgs mass points produce very similar histograms. The properties of maximum likelihood fitting ensure the distribution of most likely values is Gaussian [46]. The subsample size of 500 does not affect the mean of the $S_{m}$ distribution, and so does not affect the normalization constant obtained. 


\begin{tabular}{cc}
\hline Matrix Element & Normalization $\left(\sigma_{t \bar{t}} / \sigma(\boldsymbol{\Theta})\right)$ \\
\hline$t \bar{t}$ & 1 \\
$Z J J$ & $1.90 \times 10^{-2}$ \\
$Z Z$ & $8.90 \times 10^{-4}$ \\
$Z H 100$ & $1.77 \times 10^{3}$ \\
$Z H 105$ & $2.01 \times 10^{3}$ \\
ZH 110 & $2.15 \times 10^{3}$ \\
$Z H 115$ & $2.35 \times 10^{3}$ \\
$Z H 120$ & $2.50 \times 10^{3}$ \\
ZH125 & $2.65 \times 10^{3}$ \\
ZH130 & $2.85 \times 10^{3}$ \\
ZH135 & $3.05 \times 10^{3}$ \\
ZH140 & $3.35 \times 10^{3}$ \\
ZH145 & $3.65 \times 10^{3}$ \\
ZH150 & $4.00 \times 10^{3}$ \\
\hline
\end{tabular}

Table 6.1: Normalization constants for the matrix elements. $t \bar{t}$ was chosen as the basis for the normalization, and thus has a normalization of 1

\subsection{Pseudo-Experiments}

As described in section 6.2, pseudo-experiments are bootstrapped sets of events that follow the distributions we expect from theory (Tables 4.9 and 4.8). Because the theory predicts a constant rate of production for any given process, the number of events of events for that process must follow a Poisson distribution. The means of the Poisson distributions for each process are given in Table 4.9 and 4.8.

The SM predicts a signal $/ \sqrt{\text { background }}$ ratio of $\sim \mathscr{O}(0.1)$ (see Table 6.2). Put another way, at $m_{H}=115 \mathrm{GeV} / \mathrm{c}^{2}$ and integrated luminosity of $2.7 / \mathrm{fb}$, there is a background expectation of $\sim 300 \pm \sqrt{300}=300 \pm 17$ (stat) events and a signal expectation of $\sim$ $1.9 \pm 1.4$ (stat) events. The background fluctuations due to Poisson statistics completely overwhelm any signal that may be present.

Consider a toy example where we are fitting a one dimensional kinematic distribution with two shapes, a signal shape and a background shape. In the case of these pseudoexperiments, there would be large fluctuations in the total integrated area between 


\begin{tabular}{cccc}
$M_{H}$ & Pretag & 1 Tight & 2 Loose \\
\hline 100 & 0.051 & 0.118 & 0.161 \\
105 & 0.046 & 0.109 & 0.156 \\
110 & 0.036 & 0.085 & 0.119 \\
115 & 0.032 & 0.075 & 0.109 \\
120 & 0.029 & 0.068 & 0.097 \\
125 & 0.020 & 0.047 & 0.070 \\
130 & 0.015 & 0.034 & 0.052 \\
135 & 0.010 & 0.027 & 0.042 \\
140 & 0.008 & 0.019 & 0.028 \\
145 & 0.005 & 0.013 & 0.020 \\
150 & 0.003 & 0.007 & 0.012 \\
Table 6.2: Expected Signal/ $\sqrt{\text { Background Table }}$
\end{tabular}

pseudo-experiments. Since the signal and background shapes are fixed, there would be a large spread in the fitted parameter purely due to Poisson fluctuations.

Since the desirable outcome is to reduce the spread in the fitted parameter, we instead choose to normalize the distributions to some constant before performing the fit. This forces the fit to be based purely on shapes, and eliminates a large source of variation between pseudo-experiments. The same principle applies to the complex multidimensional fit done in this analysis.

We choose to only consider pseudo-experiments where $N_{\mathrm{PE}}=N_{\text {data }}$, where $N_{\text {data }}$ is the number of events observed at the CDF detector, in this case 290 events. This eliminates spread in the fitted parameter $S_{m}$ caused by fluctuations in pseudo-experiment size, making the analysis a pure shape fit. It also has a nice interpretation that can be thought of as a Bayesian prior, that we only consider pseudo-experiments that might be what we observed at the real experiment.

As described in Chapter 4, SECVTX $b$ tagging allows us to distinguish $b$ quark jets from other jets by looking for a secondary vertex in the silicon detector. The selection requires at least one $b$ tag, but there are kinematic differences between single $b$ tagged and double $b$ tagged events. To ensure that pseudo-experiments accurately represent the 
distributions we expect from theory, it is important to make sure the correct numbers of single $b$ tagged and double $b$ tagged events from each process are present. We define the $\zeta$ factors as

$$
\begin{aligned}
& \zeta_{1 \text { tag }}^{(j)}=N_{1 \text { tag }}^{(j)} / N^{(j)} \\
& \zeta_{2 \text { tag }}^{(j)}=N_{2 \text { tag }}^{(j)} / N^{(j)}
\end{aligned}
$$

where the index $j$ runs over the four different matrix elements (and corresponding Monte Carlo event pools), $\{Z H, Z J J, t \bar{t}, Z Z\} . \zeta$ factors are the probability of an event from a certain category being single or double tagged. Table 6.3 and 6.4 give single and double tag rates for each of the four matrix elements.

\begin{tabular}{lccc|l}
\hline & $Z J J$ & $t \bar{t}$ & $Z Z$ & \\
\hline$\zeta_{1 \text { tag }}$ & 0.91 & 0.74 & 0.80 & $\zeta_{1 \text { tag }}^{B}=0.89$ \\
$\zeta_{\text {2tag }}$ & 0.09 & 0.26 & 0.20 & $\zeta_{2 \text { tag }}^{B}=0.11$ \\
\hline
\end{tabular}

Table 6.3: Tagging rates for the backgrounds. The total $\zeta_{\mathrm{k}-\mathrm{tag}}^{B}$ are obtained by $N_{\text {back }}^{\mathrm{k}-\mathrm{tag}} /\left(N_{\text {back }}^{1 \text { tag }}+N_{\text {back }}^{\text {2tag }}\right)$ where $N_{\text {back }}^{\mathrm{k}-\mathrm{tag}}$ are the total background predictions from Table 4.9 and 4.8 and $k$ is 1 or 2 .

\begin{tabular}{lccccccccccc}
\hline $\mathrm{ZH}\left(m_{H}\right)$ & 100 & 105 & 110 & 115 & 120 & 125 & 130 & 135 & 140 & 145 & 150 \\
\hline$\zeta_{\text {tag }}$ & 0.67 & 0.67 & 0.66 & 0.66 & 0.66 & 0.65 & 0.65 & 0.64 & 0.64 & 0.64 & 0.63 \\
$\zeta_{\text {2tag }}$ & 0.33 & 0.33 & 0.34 & 0.34 & 0.34 & 0.35 & 0.35 & 0.36 & 0.36 & 0.36 & 0.37 \\
\hline
\end{tabular}

Table 6.4: Tagging rates for the signal at all Higgs masses between 100 and $150 \mathrm{GeV} / \mathrm{c}^{2}$.

In addition to Poisson variability, each process has an associated rate systematic uncertainty from things like $k$-factor, $b$ tagging uncertainties, and luminosity. Each rate systematic uncertainty is independent from other rate systematics, but is correlated across production processes. So for each production process, we add the different systematics in quadrature (because they are independent) and correlate the same systematic across processes. Therefore, all of the rate systematics can be reduced to a single distribution that is scaled according to the process. We believe the distribution to be Gaussian with a 
mean of zero and width $(\sigma)$ as given in Table 6.5. A detailed examination of factors that compose the systematic are in section 6.7.

\begin{tabular}{lr}
\hline Process & Scale Factor \\
\hline$Z \rightarrow l^{+} l^{-}+u \bar{u}, d \bar{d}, s \bar{s}$ & 0.425 \\
$Z \rightarrow l^{+} l^{-}+c \bar{c}, b \bar{b}$ & 0.435 \\
$W Z, Z Z$ & 0.246 \\
QCD & 0.500 \\
$t \bar{t} \rightarrow l \bar{l} \nu \bar{\nu} b \bar{b}$ & 0.224 \\
\hline Table 6.5: Rate Systematic scale factor
\end{tabular}

Since we are searching for $Z H$ signal in a shape fit, the amount of $Z H$ in the pseudoexperiment must be a parameter of construction. This parameter is called $S_{\text {true }}=S_{t}=$ $N_{\mathrm{ZH}} / N_{\mathrm{PE}}$ and represents the (known) fraction of $\mathrm{ZH}$ signal in the pseudo-experiment, where $N_{Z H}$ is the poisson mean. Now we are ready to follow the pseudo-experiment procedure.

Pseudo-experiment Procedure

To understand the systematics, please see section 6.7.

1. Calculate the number of signal and background events expected given $S_{t}$.

$$
N_{s}=S_{t} N_{\text {data }} \quad N_{b}=N_{\text {data }}-N_{s}
$$

2. Calculate the breakdown of signal and background events into 1-tag and 2-tag channels

$$
\begin{array}{ll}
N_{s}^{(1 \operatorname{tag})}=\zeta_{1 \mathrm{tag}}^{(\mathrm{ZH})} N_{s} & N_{s}^{(2 \operatorname{tag})}=\zeta_{2 \operatorname{tag}}^{(\mathrm{ZH})} N_{s} \\
N_{b}^{(1 \mathrm{tag})}=\zeta_{1 \mathrm{tag}}^{B} N_{b} & N_{b}^{(2 \operatorname{tag})}=\zeta_{2 \operatorname{tag}}^{B} N_{b}
\end{array}
$$


3. Calculate event 1 tag and 2 tag probabilities and signal fractions

$$
\begin{gathered}
P_{1 \mathrm{tag}}=\left(N_{s}^{(1 \mathrm{tag})}+N_{b}^{(1 \mathrm{tag})}\right) / N_{\text {data }} \\
P_{2 \mathrm{tag}}=\left(N_{s}^{(2 \mathrm{tag})}+N_{b}^{(2 \mathrm{tag})}\right) / N_{\text {data }} \\
S_{t}^{(1 \mathrm{tag})}=N_{s}^{(1 \mathrm{tag})} /\left(N_{s}^{(1 \mathrm{tag})}+N_{b}^{(1 \mathrm{tag})}\right) \\
S_{t}^{(2 \mathrm{tag})}=N_{s}^{(2 \mathrm{tag})} /\left(N_{s}^{(2 \mathrm{tag})}+N_{b}^{(2 \mathrm{tag})}\right)
\end{gathered}
$$

4. Generate a Binomial variate with $p=P_{1 \mathrm{tag}}, q=P_{2 \mathrm{tag}}$, and $N=N_{\mathrm{data}}=290$. This will give the single tagged PE size and the double tagged PE size by subtraction from $N_{\text {data }}$. This gives $N_{\text {constraint }}^{(1 \mathrm{tag})}$ and $N_{\text {constraint }}^{(2 \mathrm{tag})}$ respectively. Clearly $N_{\text {constraint }}$ fluctuates across different PEs.

5. Generate a Gaussian $(\mu=0, \sigma=1)$ variate for rate scaling. For each process, scale this variate by the quadrature sum of all rate systematics (dominated by the $k$-factor, see Table 6.5). Scale all background poisson means from Table 4.9 and 4.8 by the scaled Gaussian variate.

6. Generate Poisson variates per process based on the scaled poisson means in the 1 tag and 2 tag channel separately. Independently check that the number of events in the 1 tag channel $=N_{\text {constraint }}^{(1 \text { tag }}$ and the number of events in the 2 tag channel $=N_{\text {constraint }}^{(2 \operatorname{tag})}$. If the constraint is not met for the 1 tag or 2 tag channel, generate a new set of Poisson variates for all processes within the tag channel. This is equivalent to discarding PEs where $N \neq N_{\text {constraint }}$. Because $N_{\text {data }}$ is the sum of the two $N_{\text {constraint }}$, this implies PEs where $N \neq N_{\text {data }}$ are discarded.

7. Some Gaussian variates are heavily suppressed, (with a k-factor of 0.4 , if we fluctuate $2.5 \sigma$ low, the poisson mean is zero), so we limit the number of sets of Poisson variates for a given Gaussian variate to 1000 . The limit changes by a maximum of 
$0.5 \%$ as the number of Poisson sets per Gaussian variate varies from 1 to $10^{6}$, which is significantly less than the statistical error on the limit.

8. At this point there is a list of channels and processes $(9$ background and 1 signal process, 2 tag channels)=20 with a number of events to draw from each. We go to the pools of events (which have weights from trigger efficiencies and cross section), and using rejection based Monte Carlo, we draw the appropriate number of events from each category. These events form a pseudo-experiment, which is then fit using the likelihood fitter.

All tagging rates and $\zeta$ factors are based on Monte Carlo, not observed fluctuations in the data. This makes the analysis 'blind' to the observed number of 1 tag and 2 tag events. This is desirable as poisson $b$ tagging fluctuations would propagate to a better or worse limit otherwise, amplifying $b$ tagging uncertainty in the process.

\subsection{Likelihood Fitting - 2}

From Table 6.2, it is clear that the $S / \sqrt{B}$ is significantly higher in the 2 tag channel than in the 1 tag channel. We seek to take advantage of this to improve sensitivity by separating the two tag channels. However, for illustrative purposes, first consider the simpler case presented in section 6.1 (Combined tags).

\subsubsection{Combined Tag Channels}

We reproduce equation 6.2 here

$$
\mathscr{L}(S)=\prod_{i} L\left(S, \mathbf{x}_{i}\right)=\prod_{i} S\left[P_{s}\left(\mathbf{x}_{i} \mid \boldsymbol{\Theta}\right)+(1-S) P_{b}\left(\mathbf{x}_{i} \mid \boldsymbol{\Theta}\right)\right]
$$

To account for multiple backgrounds we replace $P_{b}$ with a correctly weighted sum of the matrix element density functions for the backgrounds, namely

$$
P_{b}\left(\mathbf{x}_{i} \mid \Theta\right) \rightarrow \lambda_{1} P_{b 1}\left(\mathbf{x}_{i} \mid \Theta\right)+\lambda_{2} P_{b 2}\left(\mathbf{x}_{i} \mid \Theta\right)+\lambda_{3} P_{b 3}\left(\mathbf{x}_{i} \mid \Theta\right)+\ldots
$$


where $\lambda_{j}$ is the background fraction of the $j$ th background as defined by $\lambda_{j}=N_{b}^{(j)} / N_{b}$. Therefore, $\sum_{j} \lambda_{j}=1$. This constraint on $\lambda_{j}$ and the $S,(1-S)$ construction in the likelihood ensure that normalization of $\mathscr{L}$ is maintained at all $S$, an essential condition for likelihood fitting. With the three backgrounds, the likelihood expression becomes.

$$
\mathscr{L}(S)=\prod_{i}\left[\left(S P_{Z H}\left(\mathbf{x}_{i} \mid M_{H}\right)+(1-S)\left(\lambda_{Z J J} P_{Z J J}\left(\mathbf{x}_{i}\right)+\lambda_{Z Z} P_{Z Z}\left(\mathbf{x}_{i}\right)+\lambda_{t \bar{t}} P_{t \bar{t}}\left(\mathbf{x}_{i}\right)\right)\right]\right.
$$

Like the normalization case, pseudo-experiments produce a Gaussian curve in $S$, much like Figure 6.1. And like the normalization case, we make a histogram of most likely values of the signal fraction $\left(S_{m}\right)$.

\subsubsection{Separated Tag Channels}

We know that a $Z H$ signal event has a higher probability than background of being double tagged. We seek to enhance sensitivity by using this fact; we make an approximation that the tagging probability is independent of event kinematics (a reasonable approximation). Then we reweight matrix element probabilities by the tagging probability for that process.

$$
P_{j}(\mathbf{x} \mid \Theta) \rightarrow P(\mathbf{x} \mid \Theta) P_{j}(k)
$$

where $k$ is the number of tags for the event. Since $P_{j}(k)$ is exactly equivalent to the $\zeta_{j}$ factors defined earlier (equation 6.6 and 6.7), we substitute. Effectively this adds another dimension to the $P_{j}$, leading to the new matrix element expression $P_{j}(\mathbf{x}, k \mid \Theta)$. This makes equation 6.10 into

$$
\begin{aligned}
\mathscr{L}(S)=\prod_{i}[ & \left(S P_{Z H}\left(\mathbf{x}_{i}, k_{i} \mid M_{H}\right)\right. \\
& \left.+(1-S)\left(\lambda_{Z J J} P_{Z J J}\left(\mathbf{x}_{i}, k_{i}\right)+\lambda_{Z Z} P_{Z Z}\left(\mathbf{x}_{i}, k_{i}\right)+\lambda_{t \bar{t}} P_{t \bar{t}}\left(\mathbf{x}_{i}, k_{i}\right)\right)\right]
\end{aligned}
$$




\begin{tabular}{ll}
\hline$\lambda_{\text {ZJJ }}$ & 0.8852 \\
$\lambda_{\text {ZZ }}$ & 0.0463 \\
$\lambda_{t \bar{t}}$ & 0.0685 \\
\hline
\end{tabular}

Table 6.6: $\lambda$ background fractions are based on theory predictions, as given in Tables 4.9 and 4.8

which coupled with tables 6.3, 6.4, and 6.6, completely defines the likelihood fitter.

To allow for statistical fluctuations in the fitted signal fraction we scan outside the physical $[0,1]$ region. This occasionally leads to difficulties in finding the likelihood peak in an automated way in the analysis software.

$$
\begin{aligned}
\log \mathscr{L}(S)=\sum_{i} \log \left[\left(S P_{Z H}\left(\mathbf{x}_{i}, k_{i} \mid M_{H}\right)\right.\right. \\
\left.+(1-S)\left(\lambda_{Z J J} P_{Z J J}\left(\mathbf{x}_{i}, k_{i}\right)+\lambda_{Z Z} P_{Z Z}\left(\mathbf{x}_{i}, k_{i}\right)+\lambda_{t \bar{t}} P_{t \bar{t}}\left(\mathbf{x}_{i}, k_{i}\right)\right)\right]
\end{aligned}
$$

When fitting the peak, we use the log likelihood, as in equation 6.13. Reliably finding the peak is not always trivial, so we use the well known and efficient Nelder-Mead $[50,51]$ maximization algorithm. Nelder-Mead, also known as the amoeba method, is preferred over other methods as it does not require the calculation of derivatives and minimizes computationally expensive function evaluations of likelihood. The specific implementation used is from the Apache Java Commons Math 2.0 Snapshot.

\subsection{Feldman-Cousins Method}

The likelihood fitter produces a likelihood curve for each constructed pseudo-experiment. Examples for pseudo-experiment likelihood and log-likelihood curves are shown as Figures 6.3 and 6.4 .

The most likely value is the $S_{\text {measured }}$, the measured signal fraction, for a given $S_{\text {true }}$, the true signal fraction the pseudo-experiment was constructed with. We map the correspondence between the true signal fraction and the measured signal fraction, and 


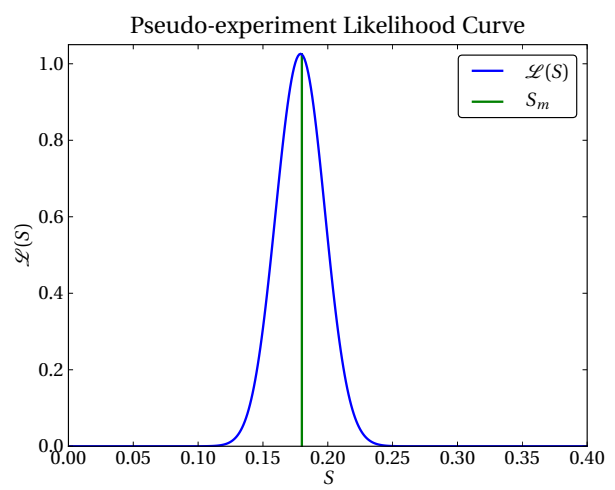

FIGURE 6.3: Pseudo-experiment Likelihood

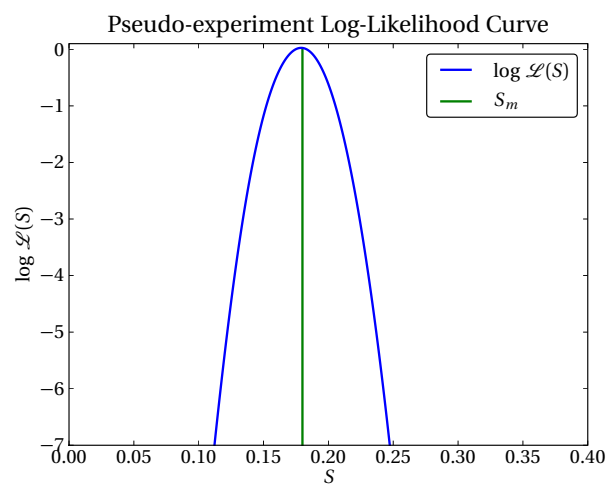

FIGURE 6.4: Pseudo-experiment LogLikelihood

the mapping allows us to adjust for any biases in the estimator.

The Feldman-Cousins method [52] provides a prescription for performing this mapping and setting a confidence interval. It requires scanning in the theory parameter, and forming a distribution of the measured parameter on simulated pseudo-experiments. The relationship can be inverted to obtain the distribution in the theory parameter given the measured parameter. The most likely theory parameter for any given $S_{\text {measured }}$ is $S_{\text {true }}^{\text {best }}$.

$$
R=\frac{\mathscr{H}\left(S_{m} \mid S_{t}\right)}{\mathscr{H}\left(S_{m} \mid S_{t}^{\text {best }}\right)}
$$

The ratio $R$ gives the relative likelihood of two hypotheses, one with a theory parameter of $S_{t}$ and the other with a theory parameter of $S_{t}^{\text {best }}$. Obviously when $S_{t}=S_{t}^{\text {best }}, R=1$, otherwise $R<1 . R\left(S_{t} \mid S_{m}\right)$ can be be thought of as the probability density function in $S_{t}$ for a given $S_{m}$.

The ratio of the relative likelihoods (not the same likelihood as the matrix element likelihood) is something known as a likelihood ratio test, which is equivalent to more well known statistical hypothesis tests like a $t$-test. Choosing a value of the ratio $R$ is equivalent to choosing a statistical significance confidence level.

The ratio $R$ is used as the test statistic in the Feldman-Cousins procedure, and the frequentist Neyman construction is used to form confidence bands. This ensures statistical 


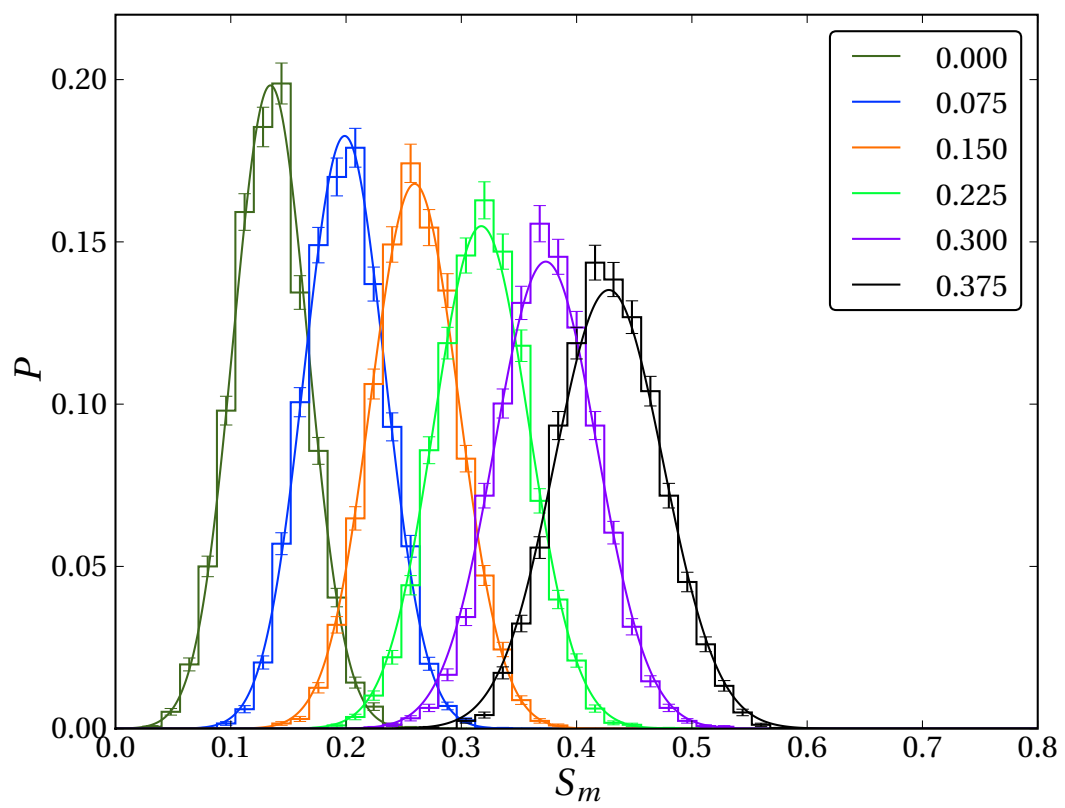

FIGURE 6.5: Measured Signal Fraction Peak Histograms. The legend indicates $S_{t}$.

coverage by construction and allows a self-consistent way of setting a two sided or one sided coverage interval.

We follow the Feldman-Cousins procedure for this analysis. Figure 6.5 shows the histograms $\mathscr{H}\left(S_{m} \mid S_{t}\right)$ for selected $S_{t}$ values in the scan. From the properties of likelihood fitting [46], we know that $S_{m}$ has a Gaussian distribution as long as the $P_{s}(\mathbf{x})$ and $P_{b}(\mathbf{x})$ have compact support. We fit Gaussians to the histograms and use the fitted Gaussians (shown) as the distribution of $S_{m}$, denoted by $\mathscr{H}\left(S_{m} \mid S_{t}\right)$.

Noticeably, the width of the fitted Gaussians increase as the amount of average signal fraction present in pseudo-experiments $\left(S_{t}\right)$ increases. This is exactly what we expect if we think of the likelihood fit as a two component signal-background fit, because the size of the Poisson fluctuations on signal increase as $S_{t}$ increases. We gain a better sense of what the histograms look like in two dimensions (Figure 6.6). The figure indicates that the mean and spread of $\mathscr{H}\left(S_{m}\right)$ can be modeled as a function of $S_{t}$. We fit an ad-hoc cubic polynomial for the mean and width of $\mathscr{H}$. 
The polynomial fit for the mean and width of $\mathscr{H}\left(S_{m}\right)$ gives an analytic model of $H\left(S_{m} \mid S_{t}\right)$. We then take the ratio $R$ as defined in equation 6.14 and normalize the ratio distribution for each $S_{t}$. In the $S_{m}, S_{t}$ plane, this leads to adjusted distribution of Figure 6.7.

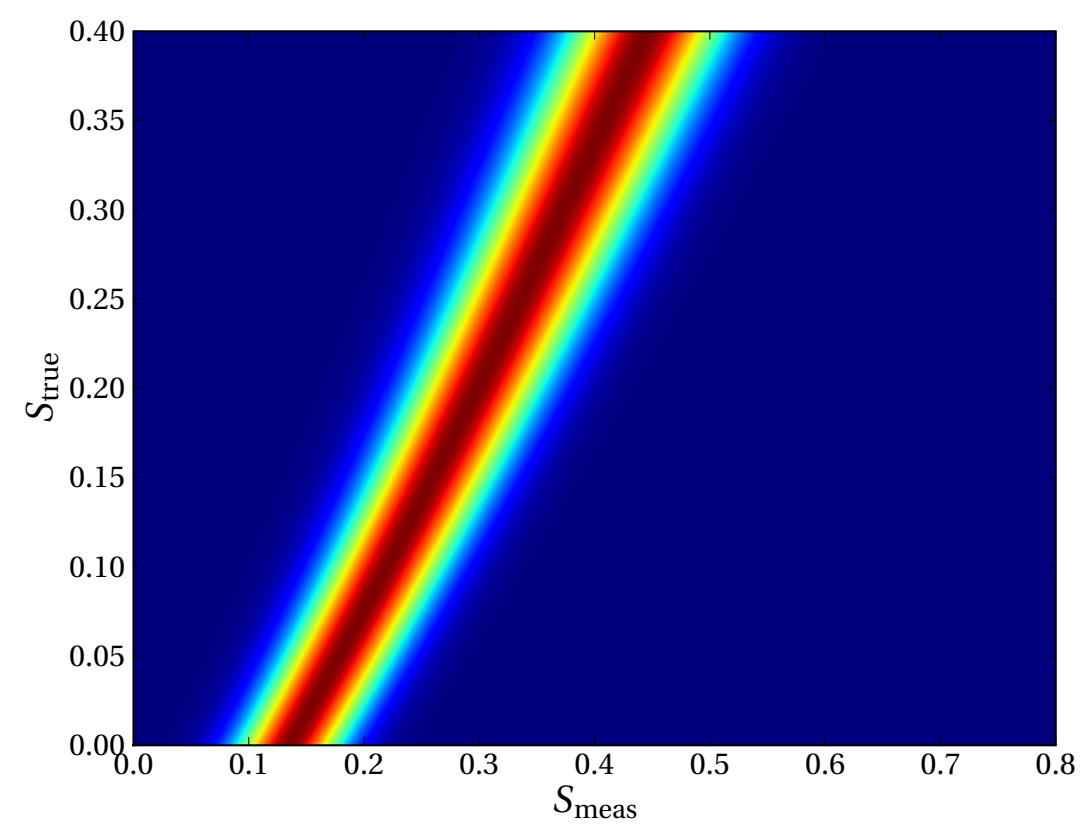

FIGURE 6.6: Measured Signal Fraction 2D Map. Warmer colors indicate higher probabilities of measurement.

For a given $S_{t}$ and coverage threshold, we select the central interval in $R$ such that the integrated area is equal to the coverage threshold. We do this at all $S_{t}$ to get the boundaries at the specified coverage interval. This sets the coverage bands over the $S_{m}, S_{t}$ plane which is all that is necessary for setting a one-sided or two-sided interval using the Feldman-Cousins construction. The results are presented in chapter 7. 


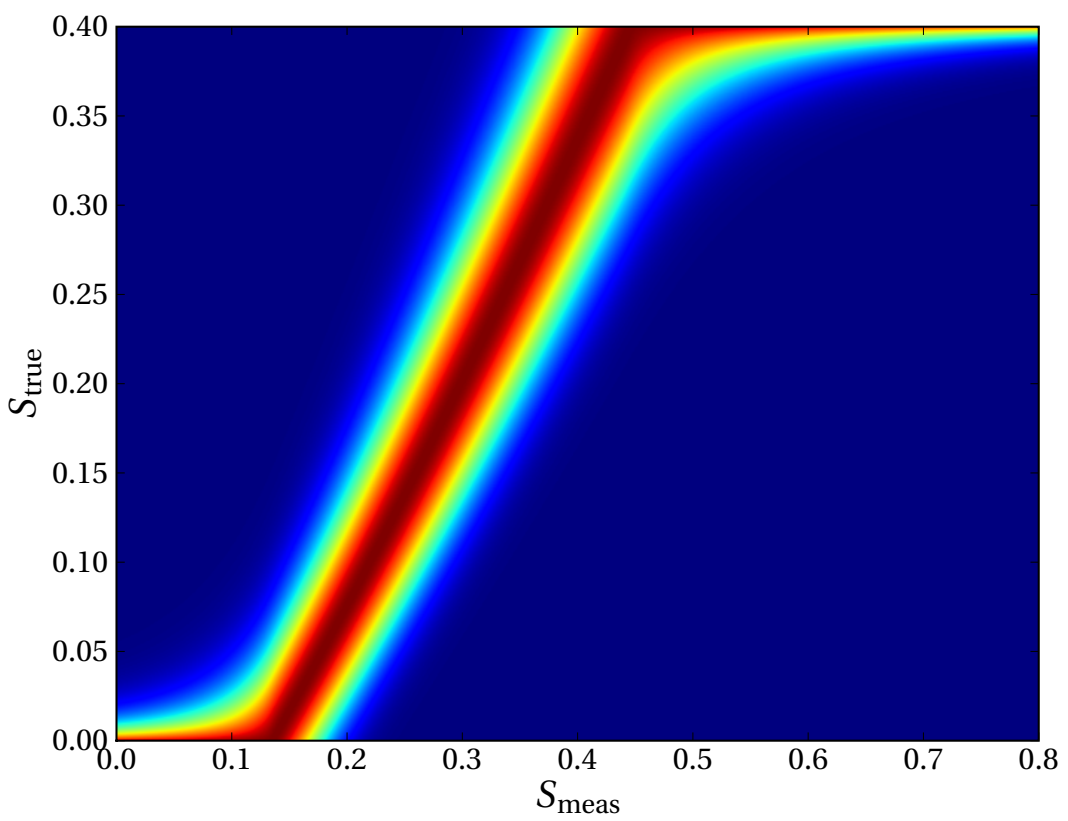

FIGURE 6.7: Feldman-Cousins Ratio

\subsection{Systematics}

There are several systematic effects that must be accounted for when performing the analysis. They can broadly be broken up into two categories, rate systematics and shape systematics. Rate systematics reflect an uncertainty in the number of events of a particular process in relation to other processes or total. Shape systematics affect the shape of the kinematic distributions.

The rate systematics are $k$-factor uncertainty, luminosity uncertainty, and $b$-Tagging uncertainty. The $k$-factor is defined as the ratio of the true cross section of a process to the leading order cross section, $k=\sigma / \sigma_{L O}$. Since the luminosity needs to be measured, there is an experimental uncertainty from the CDF luminosity detector. The $b$-tagging uncertainty indicates that the simulated tagging rate might not match the actual tagging rate. $b$-tagging uncertainty varies for the different quarks in the final state, namely that a light flavor or $c$ quark is tagged differently from an actual $b$ quark. 


\begin{tabular}{lr}
\hline Uncertainty & Value \\
\hline$k$-factor $(Z J J)$ & $40 \%$ \\
$k$-factor $(W Z / Z Z, t \bar{t})$ & $20 \%$ \\
Luminosity & $6 \%$ \\
Tagging $(u \bar{u}, d \bar{d}, s \bar{s})$ & $13 \%$ \\
Tagging $(c \bar{c})$ & $12.4 \%$ \\
Tagging $(b \bar{b})$ & $5.6 \%$ \\
\hline
\end{tabular}

Table 6.7: Rate Uncertainties. Each of the three uncertainties ( $k$-factor, Luminosity, $b$-Tagging) is believed to be independent of the others. Tagging uncertainty is applied once per tag.

Since the three rate uncertainties are independent of each other, the total uncertainty is the quadrature sum of the three uncertainties. Because each of the uncertainties is correlated across samples, the rates of all samples must be scaled simultaneously. This explains the drawing of the Gaussian variate as outlined in the pseudo-experiment construction procedure in section 6.4

The $k$-factor uncertainty dominates the rate uncertainties because of the quadrature sum. However, Table 4.8 and 4.9 show that the total number of events predicted agrees with the observed number to within $1 \sigma$. Entirely separate from the rate systematics, there are poisson fluctuations for each process. Because the analysis is a pure shape fit, the data effectively constrained the rate systematic distribution.

There are also the shape systematic uncertainties, which are Initial State Radiation (ISR), Final State Radiation (FSR), and Jet Energy Scale (JES). ISR introduces an extra parton in the initial state, leading to an extra jet in the event, $\left(q \bar{q} g \rightarrow \mathrm{ZHg} \rightarrow l^{+} l^{-} b \bar{b} g\right)$. FSR introduces an extra jet in the event as well, but in the final state $(q \bar{q} \rightarrow Z H \rightarrow$ $\left.l^{+} l^{-} b \bar{b} g\right)$. Jet Energy Scale uncertainty arises from the difficulty in calibrating the energy measured in the calorimeter with the true energy of the jet.

Shape uncertainties can be accounted for by adjusting the shape of $\mathscr{H}\left(S_{m} \mid S_{t}\right)$. For each shape systematic, we perform pseudo-experiments at $\pm 1 \sigma$ in the systematic. At any given $S_{t}$, these systematically varied pseudo-experiments lead to a shift in $\mathscr{H}\left(S_{m} \mid S_{t}\right)$ 


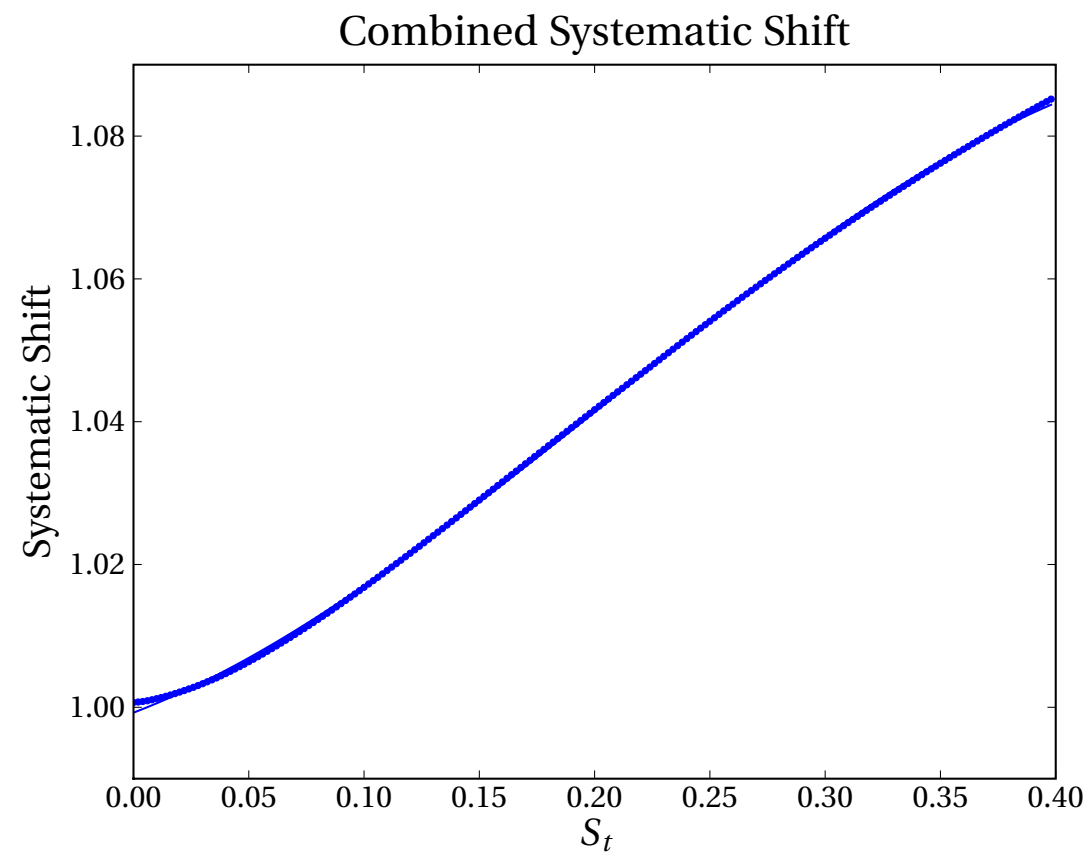

FIGURE 6.8: Rms scale factor as a function of $S_{t}$ for $S_{m}$ distributions due to shape systematics. The dominant systematic is ISR, which causes misidentification of jets when performing the selection.

mean by $\delta_{ \pm}$. We assume the shift in $\mathscr{H}\left(S_{m} \mid S_{t}\right)$ to be linear in the systematic, namely $\Delta=\delta_{ \pm} \sigma_{\text {syst }}$. Since the systematic distribution is assumed to be a Gaussian, we want to weight the shift in the $S_{m}$ histogram with a Gaussian in the systematic. Because $\mathscr{H}\left(S_{m} \mid S_{t}\right)$ is itself Gaussian, we convolute the two Gaussians,effectively adding their variances to form a new histogram $\mathscr{H}\left(S_{m} \mid S_{t}\right)$ with the systematic included. We repeat this for all three shape systematics, effectively increasing $\mathscr{H}\left(S_{m} \mid S_{t}\right)$ variance at each $S_{t}$ by the sum of the variances due to the three shape systematics. The ratio of the standard deviations with and without all systematics included is plotted in figure 6.8. It shows that over a range of 0 to $0.4 S_{t}$, standard deviation of $\mathscr{H}\left(S_{m} \mid S_{t}\right)$ is increased between 0 and $9 \%$ due to systematics. 


\section{7}

\section{Results}

Using $2.7 \mathrm{fb}^{-1}$ of data (through period 17), 290 events pass the selection outlined in chapter 4 . These 290 events are fitted with the likelihood fitter from chapter 6 . The resulting likelihood curve is Figure 7.1.

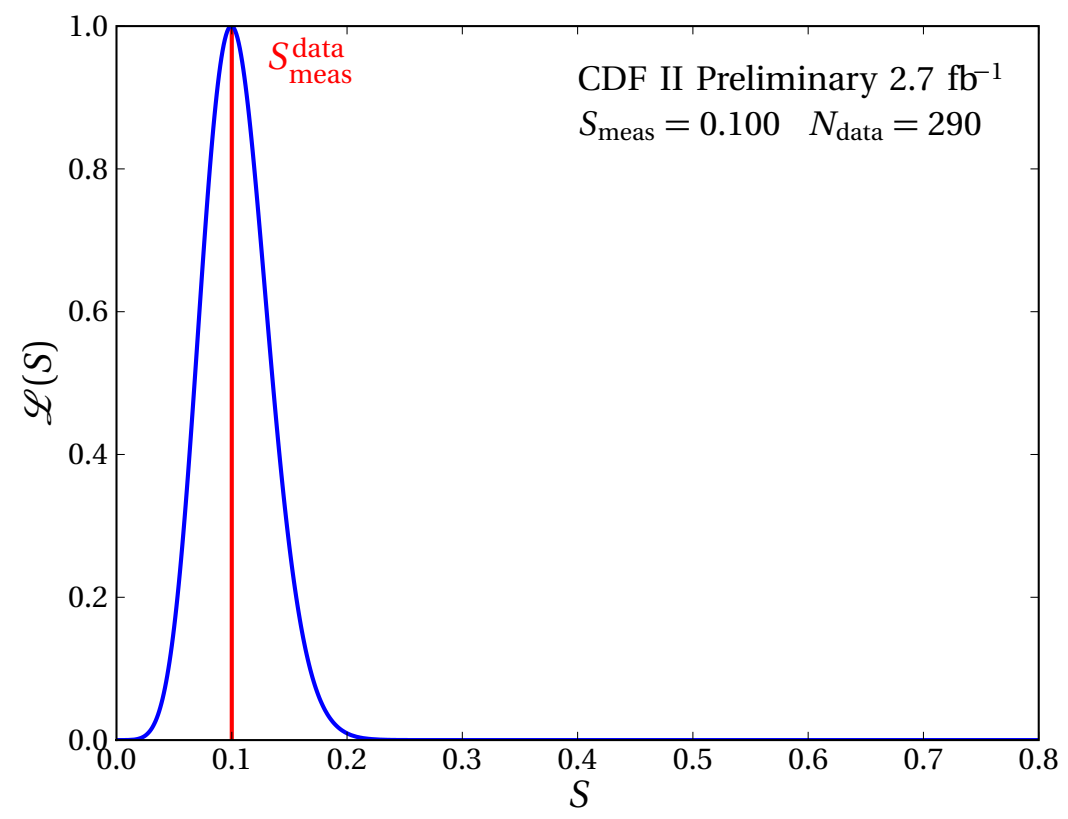

FIGURE 7.1: Data Likelihood Curve 
The data likelihood curve peaks at $S_{\text {meas }}^{(\text {data) }}=0.100$. Three discrete coverage levels corresponding to 1, 2, and $3 \sigma$ are selected from Figure 6.7 and are shown as Figure 7.2.

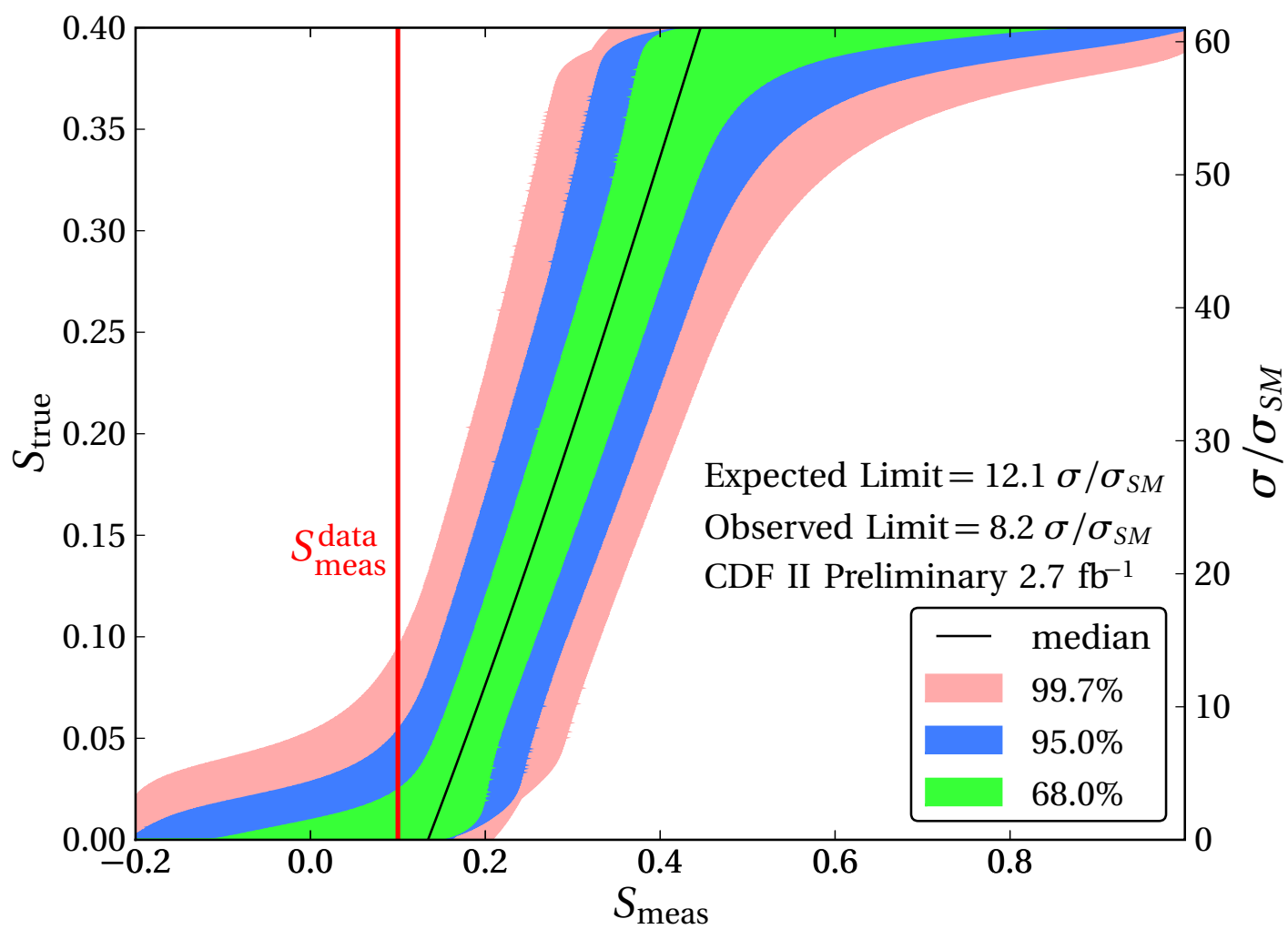

FIGURE 7.2: Feldman-Cousins confidence bands. The most likely $S$ from data is indicated as the red line. The most likely $S_{m}$ (based on simulation) as a function of $S_{t}$ is the black median line. $M_{H}=115 \mathrm{Ge} V / \mathrm{c}^{2}$

The most likely $S$ from the data is indicated as $S_{\text {meas }}^{\text {data }}$, the red line. At $95 \%$ coverage (blue band), $S_{\text {meas }}^{\text {data }}$ crosses the upper blue boundary at a $S_{\text {true }}$ of 0.0537 , which corresponds to a $\sigma / \sigma_{S M}$ of 8.2 (right axis).

The expected limit is obtained by assuming that $S_{t}=0$, and calculating the distribution of limits that would be obtained by the Feldman-Cousins prescription. The distribution for $M_{H}=115 \mathrm{GeV} / \mathrm{c}^{2}$ is given in Figure 7.3.

The limits at all masses are given in Table 7.1 using the SM cross sections defined in 


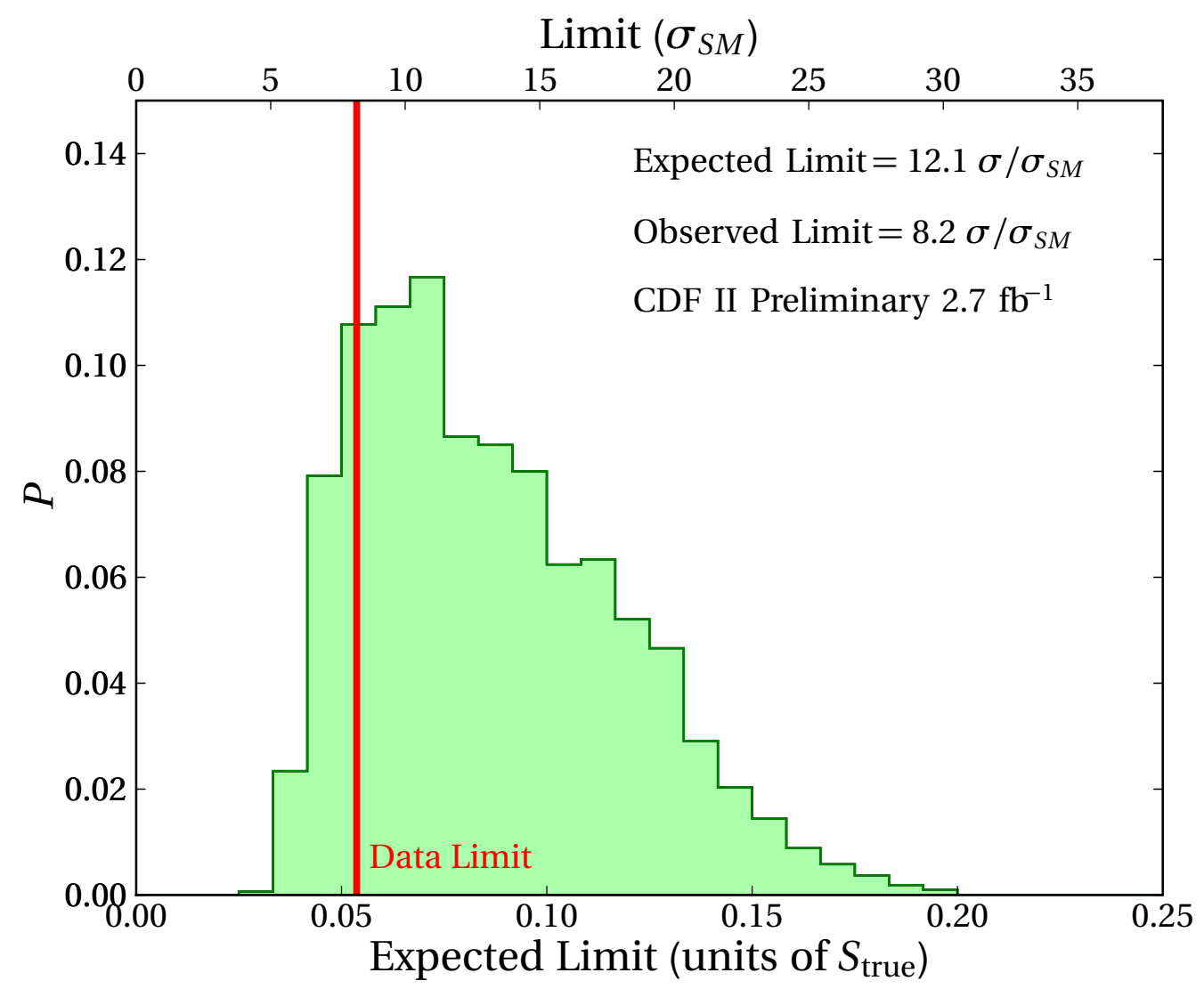

FIGURE 7.3: Expected distribution of limits assuming that $S_{t}=0$ and $95 \%$ coverage. The data limit is at the 13th percentile. The median of this distribution defines the expected limit. $\pm 1, \pm 2 \sigma$ limits are defined by the appropriate point on the cumulative density function for this distribution. $M_{H}=115 \mathrm{GeV} / \mathrm{c}^{2}$

Figure 2.14. The table is plotted in Figure 7.4. Noticeably, the data favors a lower limit as compared to the expected limit at all $M_{H}$ considered. A full set of coverage bands for all $M_{H}$ between 100 and $150 \mathrm{GeV} / \mathrm{c}^{2}$ is provided in appendix A 


\begin{tabular}{rrrrrrr}
$M_{H}$ & $-2 \sigma$ & $-1 \sigma$ & median & $+1 \sigma$ & $+2 \sigma$ & Observed \\
\hline 100 & 4.8 & 6.0 & 8.7 & 12.5 & 16.2 & 7.0 \\
105 & 4.7 & 6.1 & 8.7 & 12.9 & 17.1 & 6.5 \\
110 & 5.6 & 7.5 & 11.3 & 16.8 & 22.3 & 7.6 \\
115 & 6.4 & 8.3 & 12.1 & 18.2 & 24.2 & 8.2 \\
120 & 7.1 & 9.3 & 13.5 & 20.0 & 26.5 & 9.0 \\
125 & 10.7 & 13.2 & 18.3 & 27.1 & 35.2 & 13.2 \\
130 & 13.7 & 17.1 & 24.2 & 35.7 & 46.6 & 17.7 \\
135 & 17.4 & 21.8 & 31.0 & 44.8 & 58.6 & 22.9 \\
140 & 24.4 & 31.1 & 44.3 & 65.4 & 85.0 & 32.0 \\
145 & 33.5 & 42.8 & 61.6 & 89.9 & 118.8 & 43.2 \\
150 & 58.2 & 73.7 & 104.1 & 153.2 & 198.3 & 71.3 \\
\hline
\end{tabular}

Table 7.1: Expected and observed limits in units of $\sigma_{S M}$, as given by Figure 2.14. Uses 2.7 $\mathrm{fb}^{-1}$ of CDF II data, through period 17.

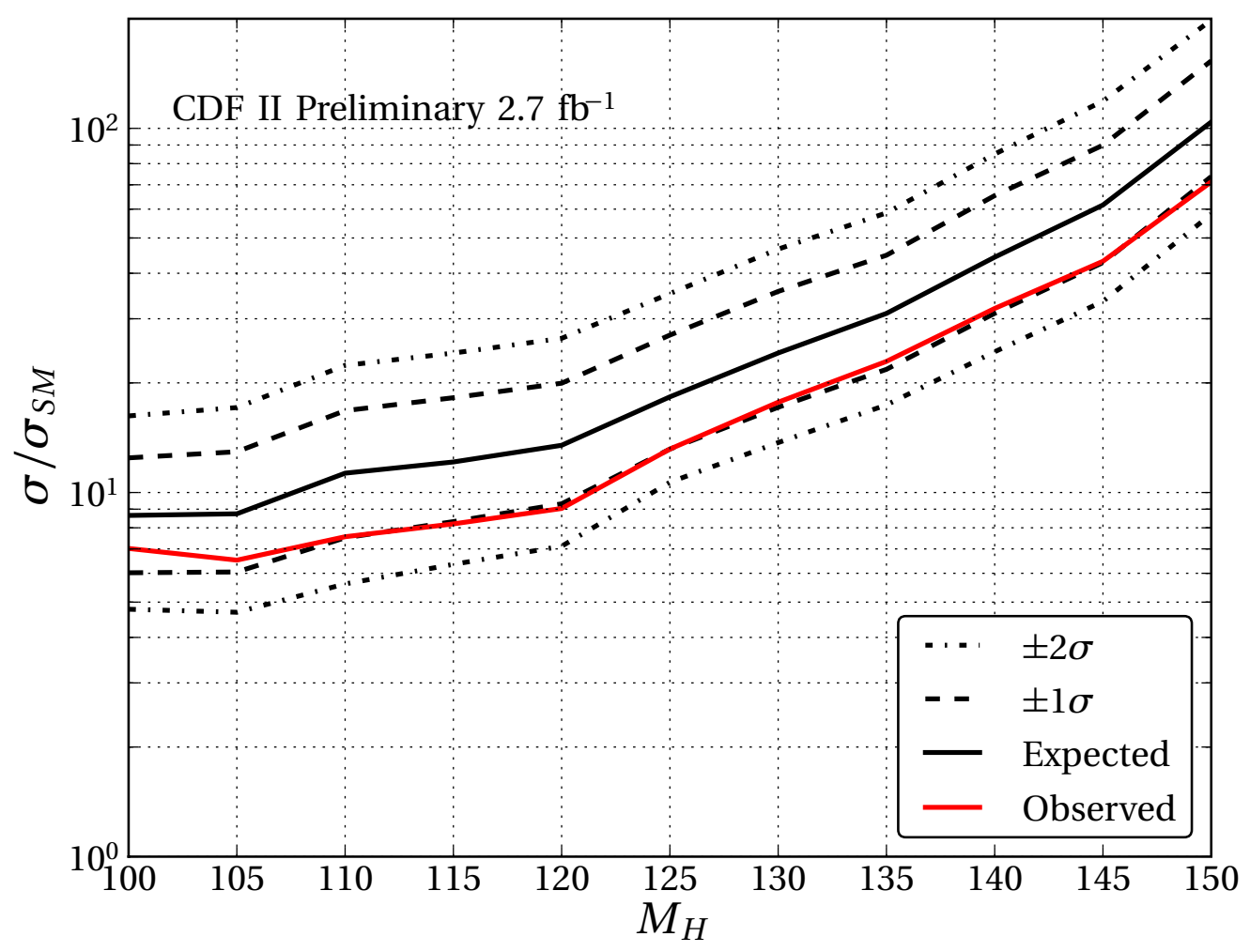

FIGURE 7.4: $p \bar{p} \rightarrow Z H \rightarrow l^{+} l^{-} b \bar{b}$ limits at $95 \%$ coverage. 


\section{8}

\section{Conclusions \& Future Work}

It is useful to consider this $Z H \rightarrow l^{+} l^{-} b \bar{b}$ analysis as part of the larger set of Higgs searches. The Tevatron continues to improve on the Higgs cross section limits (see Figure 8.1) and has even excluded a Higgs boson with a mass between $160 \mathrm{GeV} / \mathrm{c}^{2}$ and $170 \mathrm{GeV} / \mathrm{c}^{2}$ with $95 \%$ C.L. With the Large Hadron Collider due to come online in late 2009, as well as continued running of the Tevatron for lower mass Higgs searches, there are good prospects for potential discovery of the Higgs boson.

This analysis demonstrates that the matrix elements are a simple, elegant and powerful way to perform a search. In the future this technique can be extended to other analyses, or possibly other channels for the Higgs boson search. With minor changes, this technique could easily be applied to LHC data.

Currently the different Higgs search channels use neural networks with input variables not including matrix elements. A potentially large gain from adding matrix elements as neural network inputs is possible.

A genetic algorithm was tested as a way to cut the current set of (tagged) events down to a smaller set of events. It found the optimal solution to be acceptance of all the events 


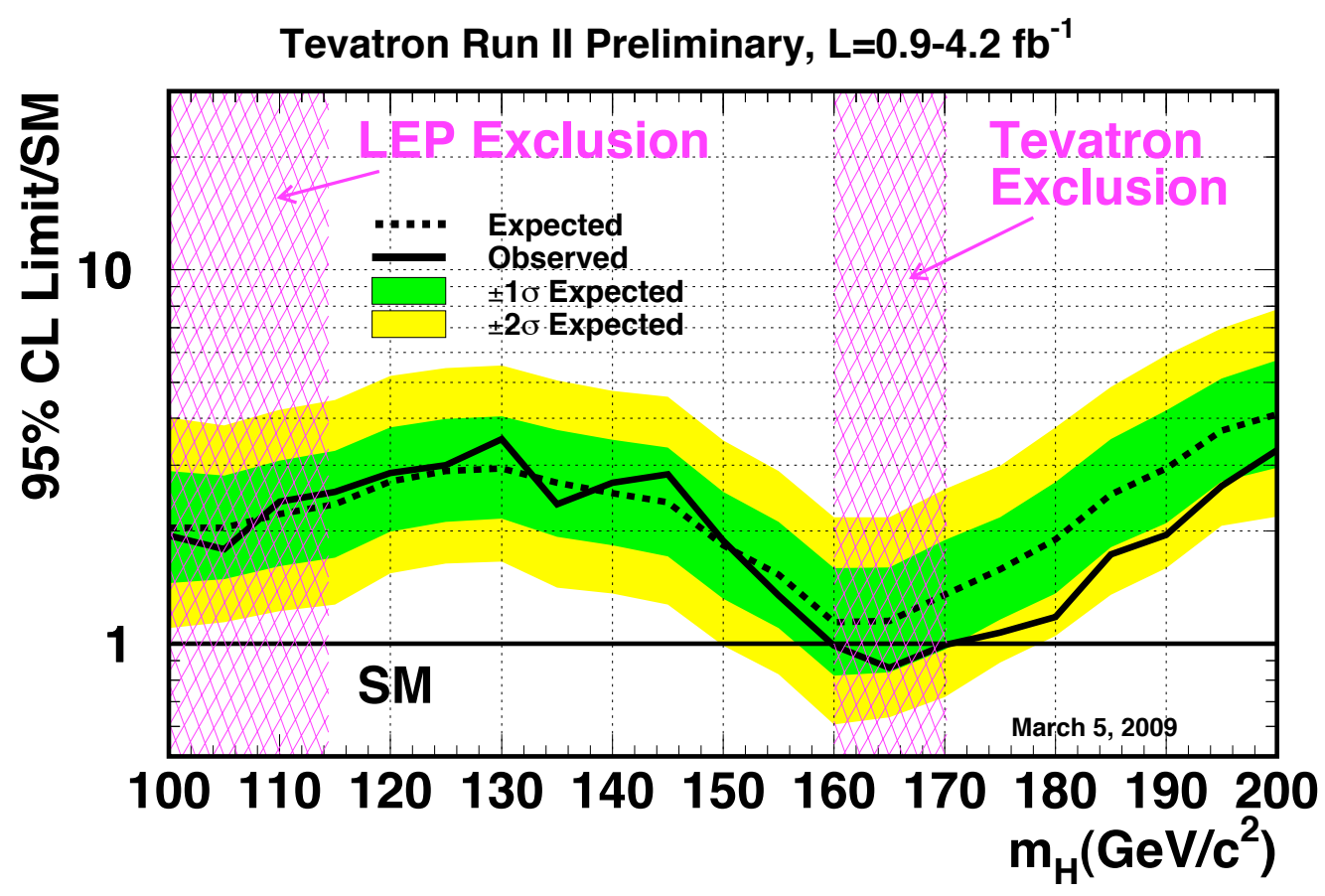

FIGURE 8.1: March 2009 Tevatron Higgs Combined Limits [53]. A Higgs boson with mass below $114 \mathrm{GeV} / \mathrm{c}^{2}$ was excluded (95\% C.L) at LEP, and masses between $160 \mathrm{GeV} / \mathrm{c}^{2}$ and $170 \mathrm{GeV} / \mathrm{c}^{2}$ have been excluded (95\% C.L.) at the Tevatron.

which are tagged. This suggests the current selection is too restrictive and there is a gain if the tagging requirements are loosened.

Unitarity of cross sections in the standard model tell us the standard model does not extend to infinite energies. Indirect constraints suggest the first evidence of phenomena beyond the SM will manifest at energy scales of $\sim \mathscr{O}(1 \mathrm{TeV})$. With the LHC partonparton collision energy approaching $1 \mathrm{TeV}$, we stand at the edge of the unknown. We either discover the Higgs boson, which would be yet another resounding success for the standard model, or discover the absence of the Higgs boson, which may be the modern version of the ultraviolet catastrophe. Regardless, it is an exciting time to research high energy physics. 


\section{Appendix A}

\section{Additional Plots}

We show data likelihood curves, Feldman-Cousins confidence bands, and expected limit distributions at all $M_{H}$ between 100 and $150 \mathrm{GeV} / \mathrm{c}^{2}$. 

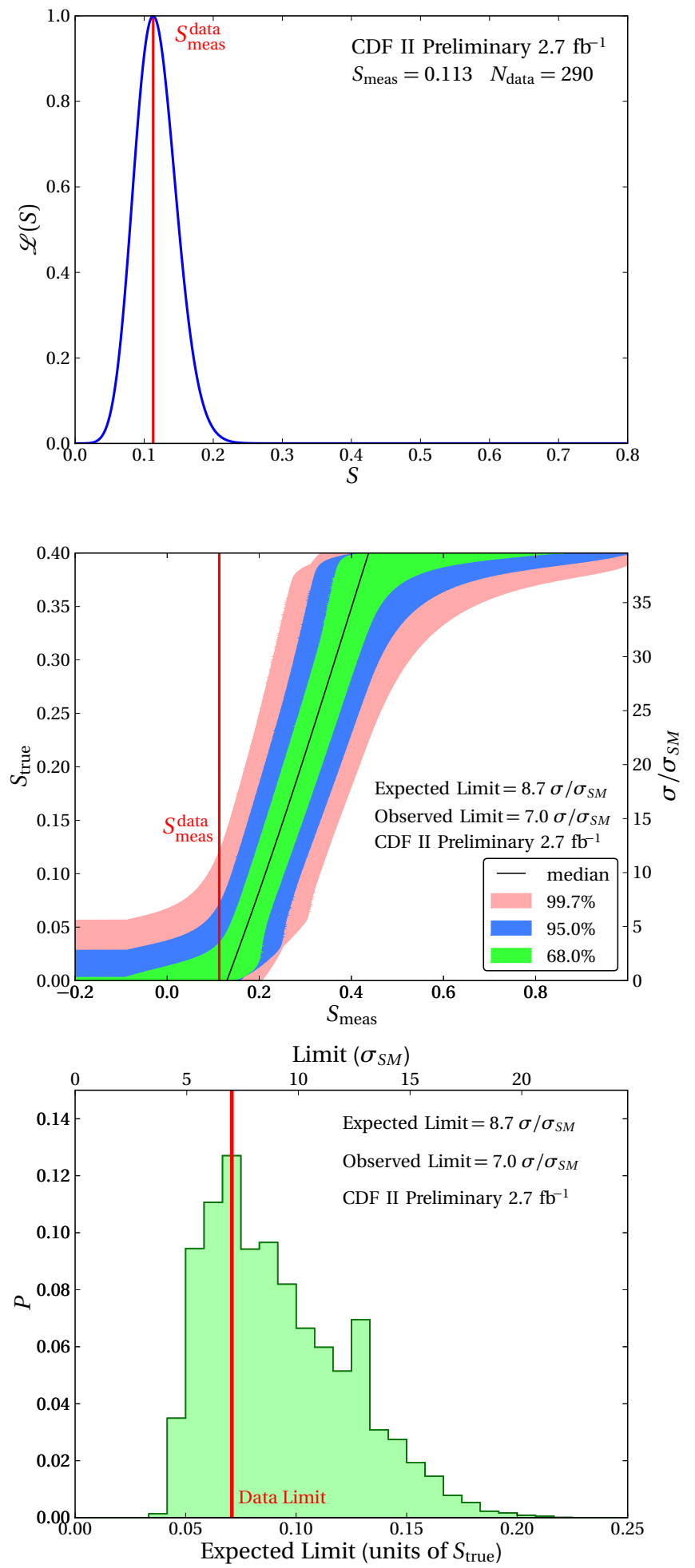

Figure A.1: $M_{H}=100 \mathrm{GeV} / \mathrm{c}^{2}$ 

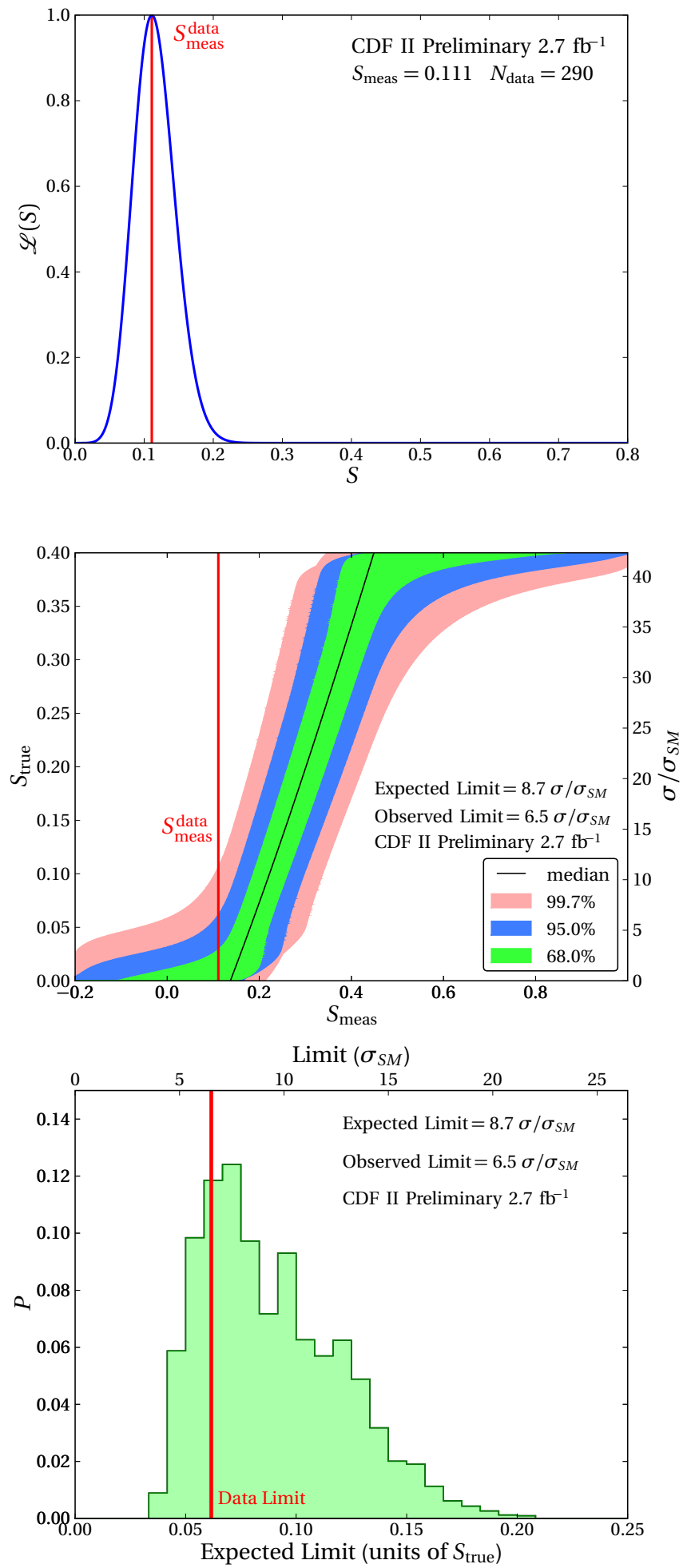

Figure A.2: $M_{H}=105 \mathrm{Ge} / \mathrm{c}^{2}$ 

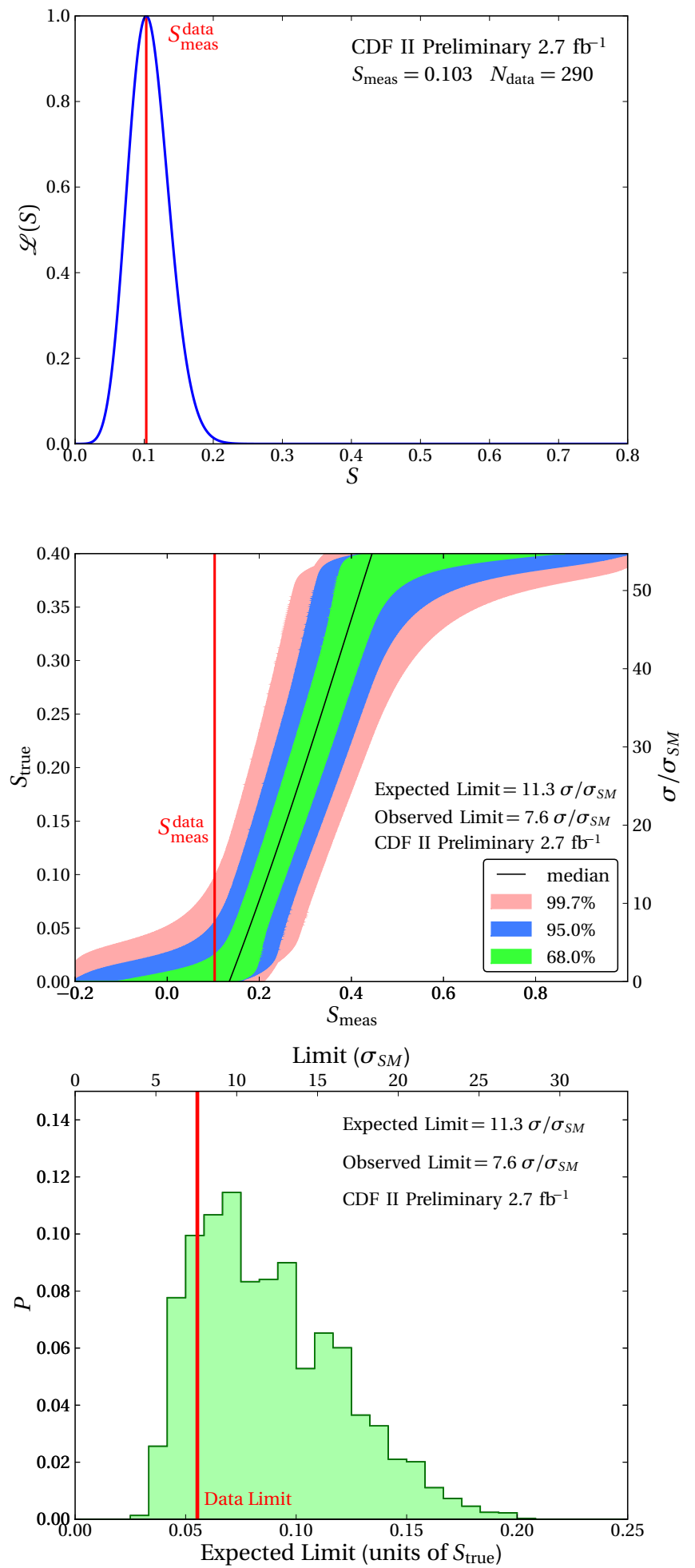

Figure A.3: $M_{H}=110 \mathrm{GeV} / \mathrm{c}^{2}$ 

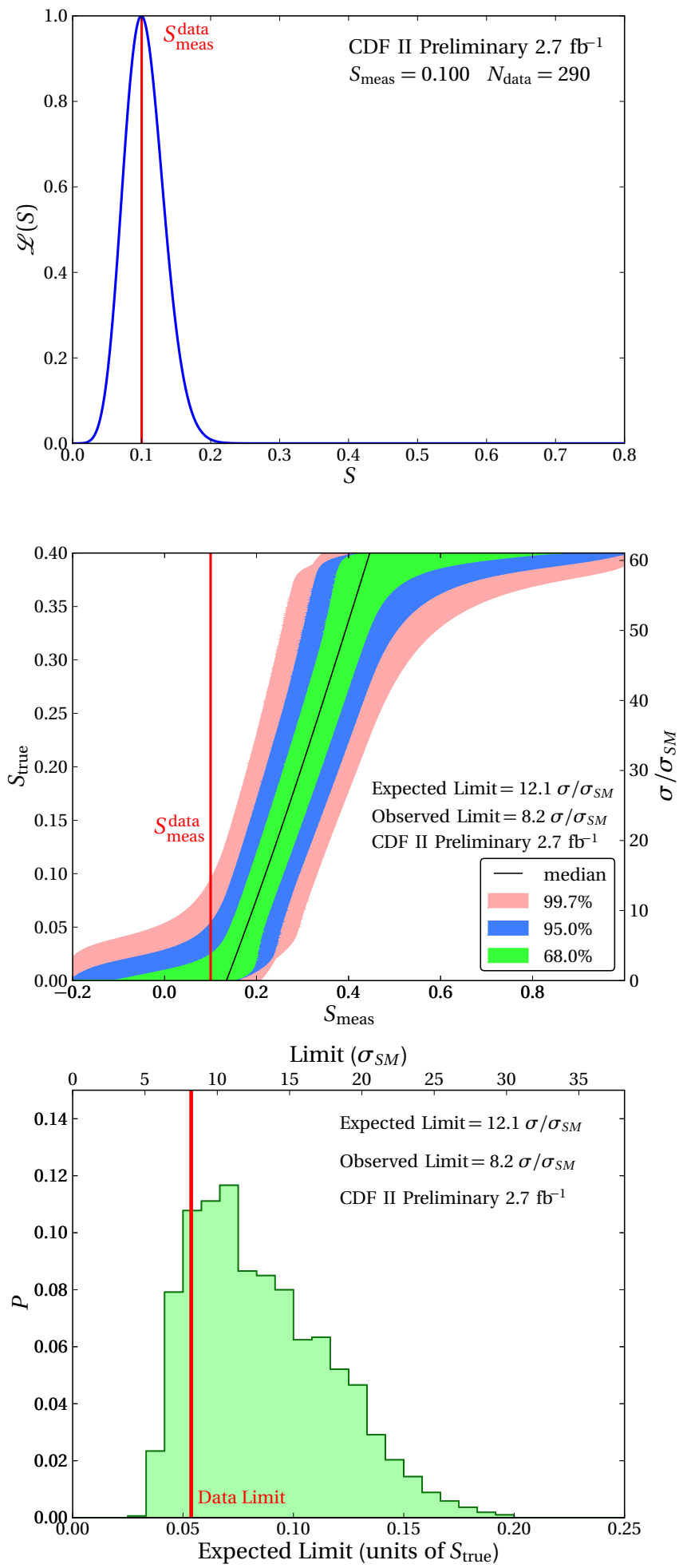

Figure A.4: $M_{H}=115 \mathrm{Ge} V / \mathrm{c}^{2}$ 

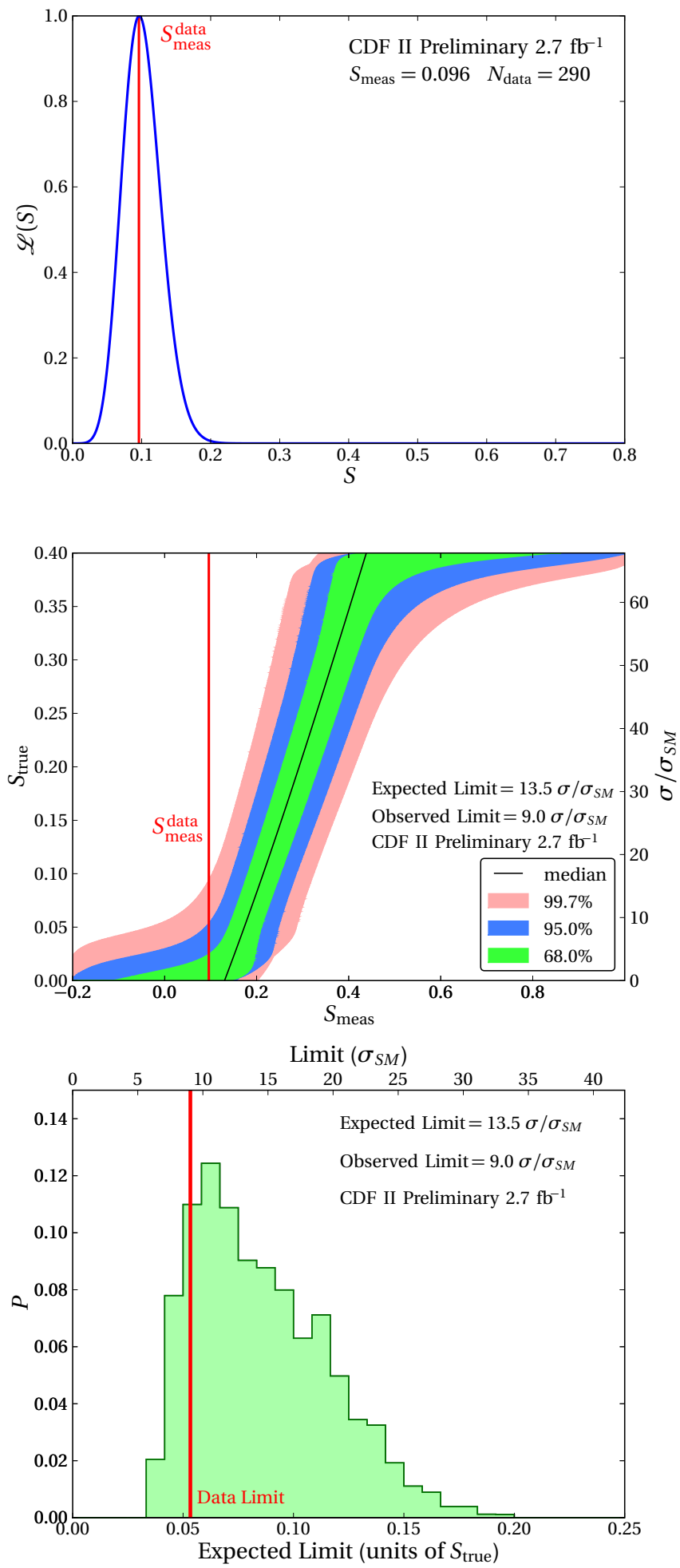

FiguRE A.5: $M_{H}=120 \mathrm{GeV} / \mathrm{c}^{2}$ 

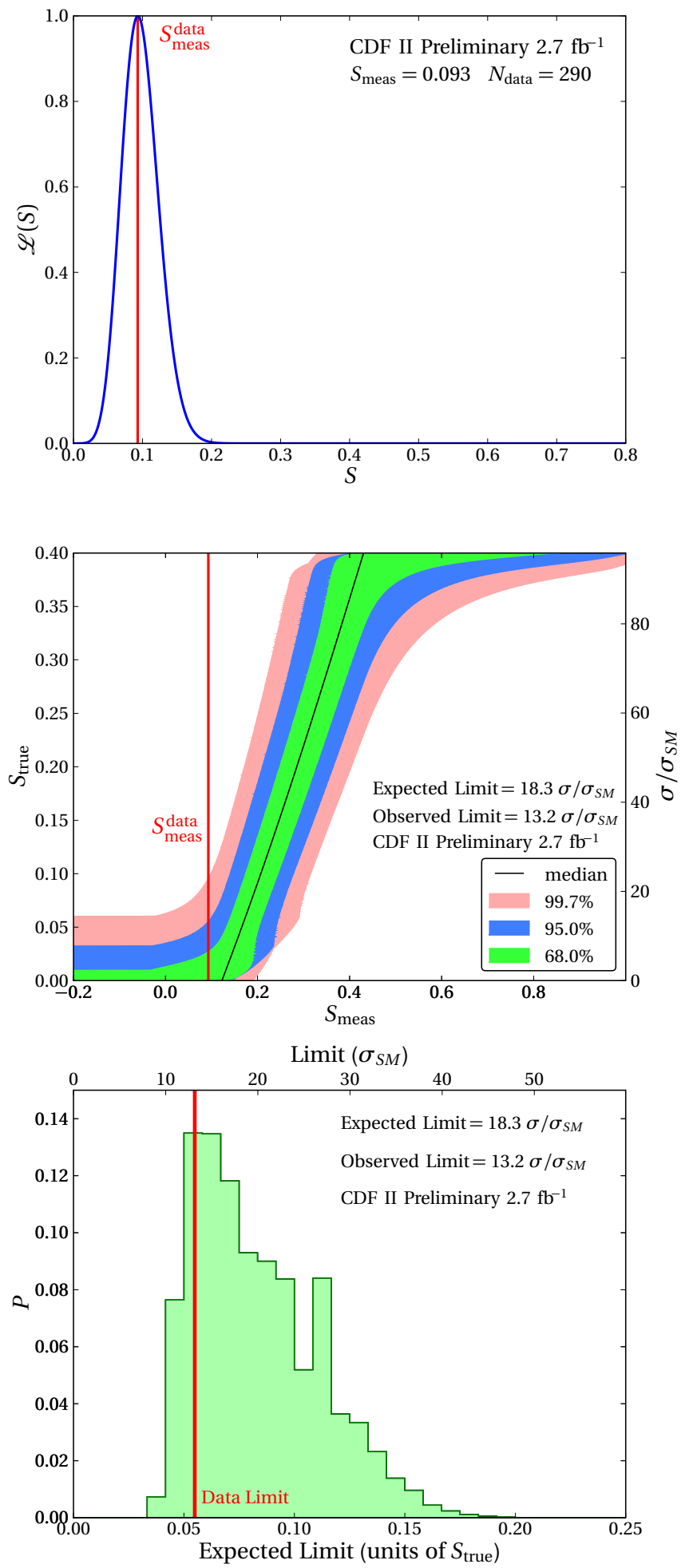

Figure A.6: $M_{H}=125 \mathrm{GeV} / \mathrm{c}^{2}$ 

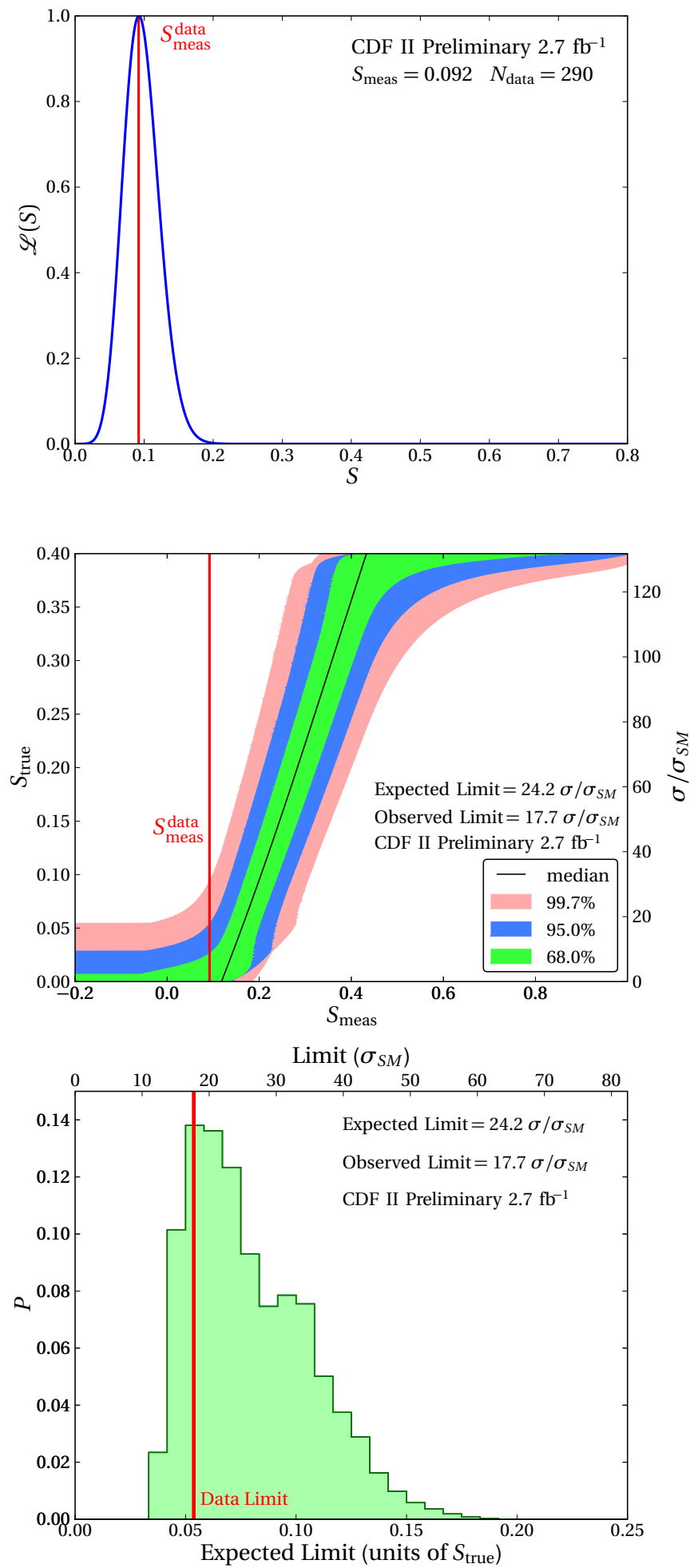

Figure A.7: $M_{H}=130 \mathrm{Ge} / \mathrm{c}^{2}$ 

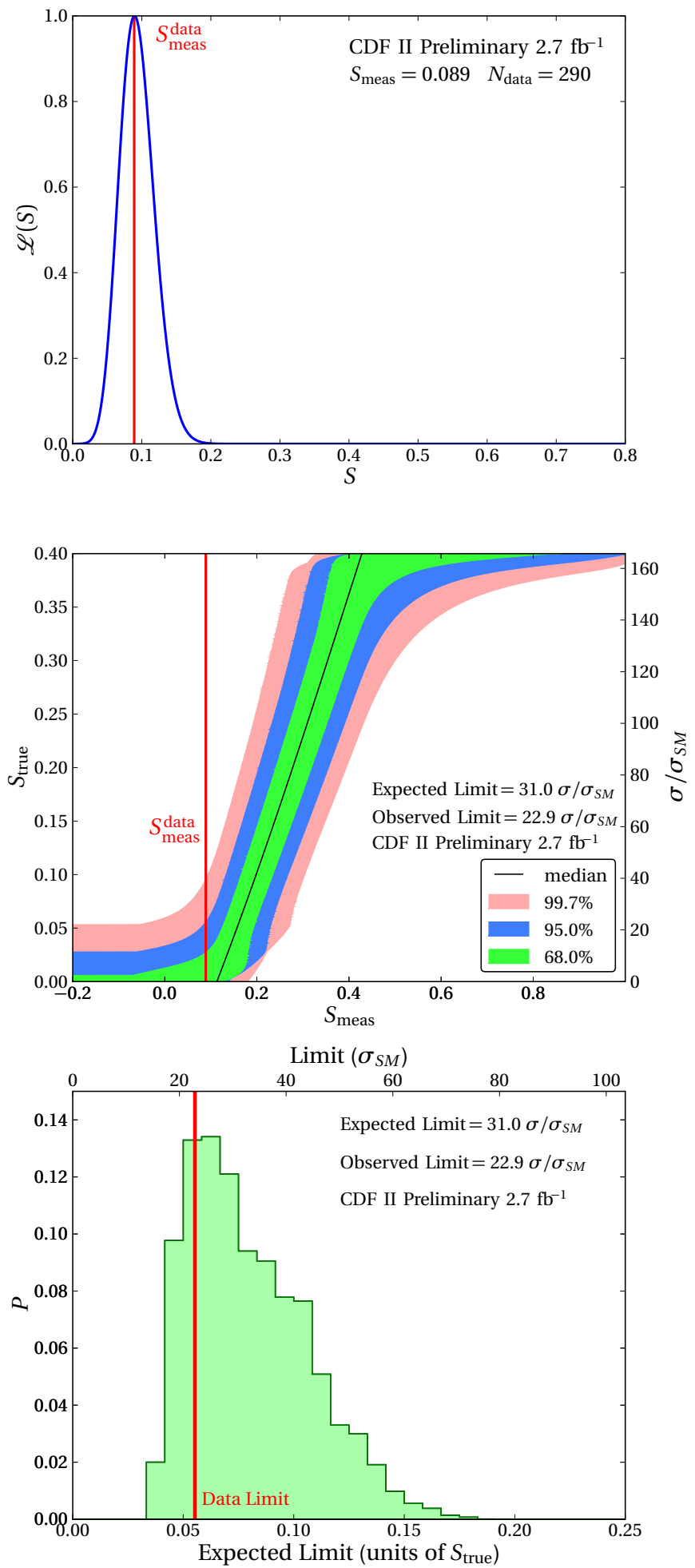

Figure A.8: $M_{H}=135 \mathrm{Ge} V / \mathrm{c}^{2}$ 

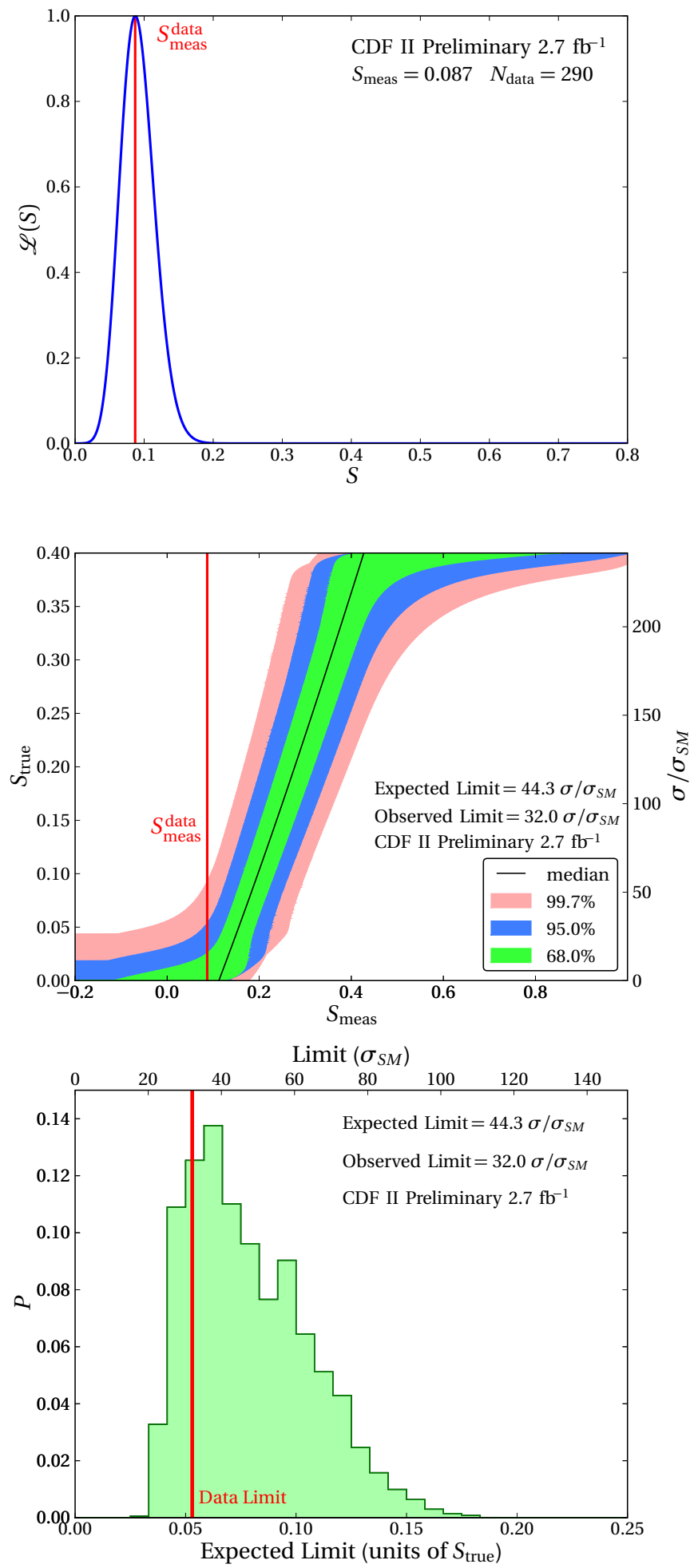

Figure A.9: $M_{H}=140 \mathrm{GeV} / \mathrm{c}^{2}$ 

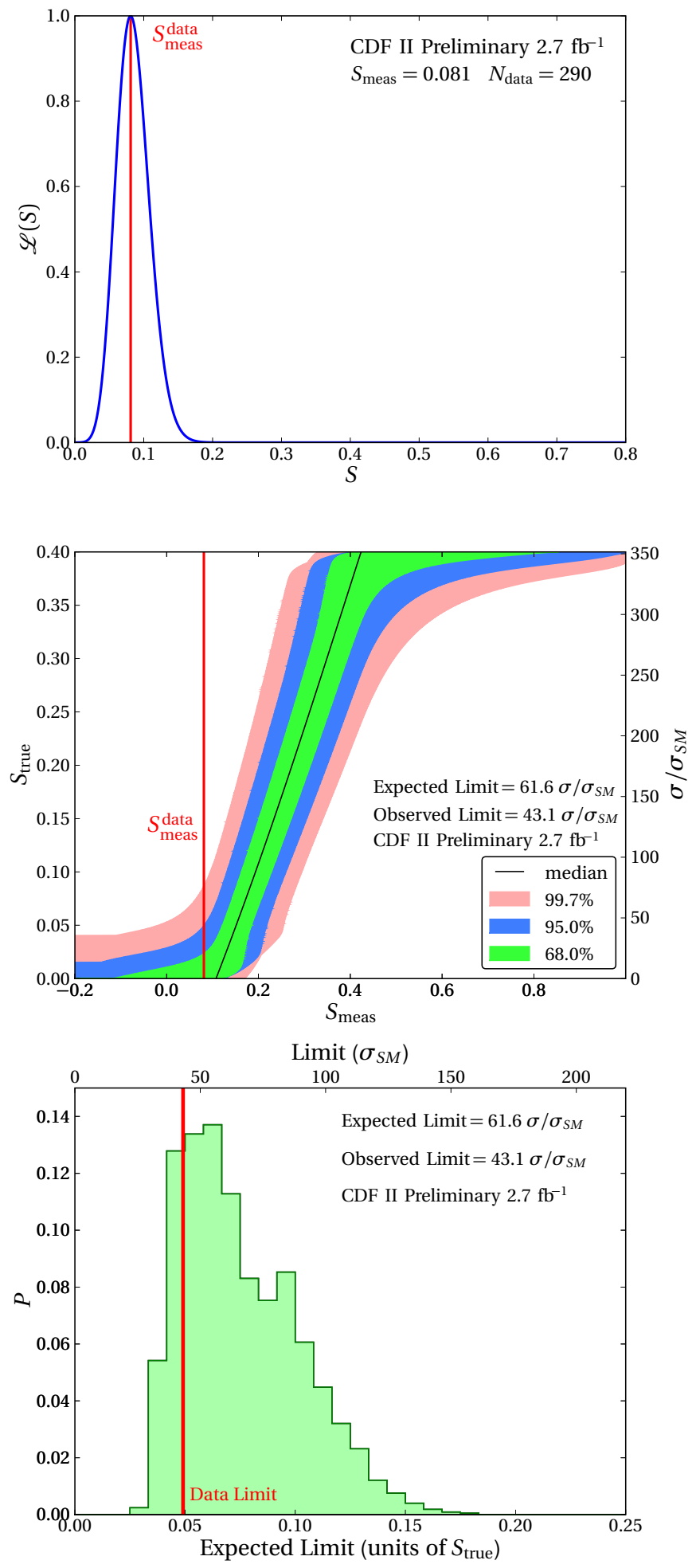

Figure A.10: $M_{H}=145 \mathrm{GeV} / \mathrm{c}^{2}$ 

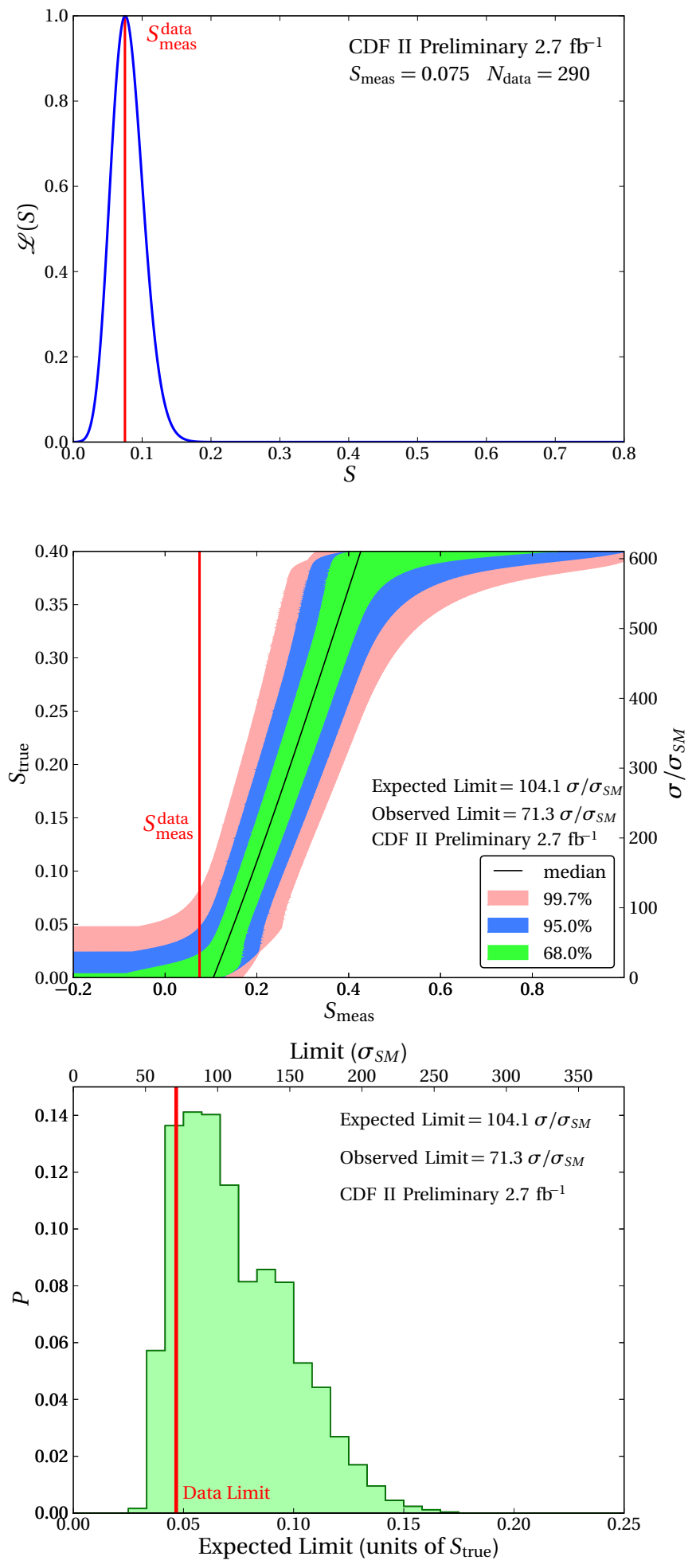

Figure A.11: $M_{H}=150 \mathrm{GeV} / \mathrm{c}^{2}$ 


\section{Bibliography}

[1] Michelson, A. Light Waves and their Uses. University of Chicago Press, Chicago, 1903.

[2] Higgs, P. Broken Symmetries and the Masses of Gange Bosons. Physical Review Letters, 13(16):508-509, 1964. doi:10.1103/PhysRevLett.13.508.

[3] SuperK Collaboration. Measurements of the Solar Nentrino Flux from SuperKamiokande's First 300 Days. Physical Review Letters, 81(6):1158-1162, 1998. doi:10.1103/PhysRevLett.81.1158.

[4] Griffiths, D. Introduction to Elementary Particles. John Willey \& Sons Inc, 1987.

[5] Wikimedia Commons. Creative Commons Attribution 3.0 Unported. Wikipedia, 2006. [Online; accessed 27-April-2009], URL http://commons . wikimedia.org/ wiki/File:Standard_Model_of_Elementary_Particles.svg.

[6] C. Amsler et al. (Particle Data Group). Review of Particle Physics. Physics Letters B, 667:1-6, 2008. doi:10.1016/j.physletb.2008.07.018. URL http://pdg. 1bl.gov.

[7] Barger, V. and Phillips, R. Collider Physics. Westview Press, 2nd edition, 1996.

[8] Aitchison, I. and Hey, A. Gauge Theories in Particle Physics, volume 1-2. Taylor and Francis Group, New York, 3rd edition, 2003.

[9] Goldstone, J. Field theories with Superconductor solutions. Il Nuovo Cimento (1955-1965), 19(1):154-164, 1961.

[10] Lee, B., Quigg, C., and Thacker, H.B. Weak Interactions at Very High Energies: The Role of the Higgs-Boson Mass. Physical Review D, 16(5):1519-1531, 1977. doi: 10.1103/PhysRevD.16.1519.

[11] Lee, B., Quigg, C., and Thacker, H.B. Strength of Weak Interactions at Very High Energies and the Higgs Boson Mass. Physical Review Letters, 38(16):883-885, 1977. doi:10.1103/PhysRevLett.38.883. 
[12] Hambye, T. and Riesselmann, K. Matching Conditions and Higgs Boson Mass Upper Bounds Reexamined. Physical Review D, 55(11):7255-7262, 1997. doi:10.1103/ PhysRevD.55.7255.

[13] Carena, M. and Haber, H. Higgs Boson Theory and Phenomenology. Progress in Particle and Nuclear Physics, 50(1):63-152, 2003.

[14] Tevatron Electroweak Working Group. Combination of CDF and DO Results on the Mass of the Top Quark. arXiv, 0903.2503, 2009. URL http://arxiv.org/abs/ 0903.2503.

[15] ALEPH, CDF, D0, DELPHI, L3, OPAL, SLD Collaborations et al. Precision Electroweak Measurements and Constraints on the Standard Model. arXiv, 0811.4682v1, 2008. URL http://arxiv.org/abs/0811.4682v1.

[16] LEPEWWG. LEP Electroweak Working Group Report. 2009. [Online; accessed 16-May-2009], URL http://lepewwg . web. cern. ch/LEPEWWG.

[17] TeV4LHC web page, 2009. [Online; accessed 5-May-2009], URL http://maltoni . home.cern.ch/maltoni/TeV4LHC.

[18] Hidas, D. Search for Standard Model Higgs Bosons Decaying to W-Boson Pairs in $p \bar{p}$ Collisions at $\sqrt{s}=1.96 \mathrm{TeV}$. CDF Note, 9734, 2007. URL http://www-cdf.fnal. gov/thesis/cdf9734_DeanHidasThesis_1.pdf\%.

[19] Fermilab Beams Division Collaboration. Tevatron Run 2 handbook. Fermilab, 1998.

[20] CDF Collaboration. The CDF detector: an overview. Nuclear Instruments and Methods in Physics Research A, 271:387.

[21] CDF Collaboration. Measurement of the J/ $\psi$ meson and b-hadron production cross sections in $p \bar{p}$ collisions at $\sqrt{s}=1960 \mathrm{GeV}$. Physical Review D, 71(3):032001, 2005. doi:10.1103/PhysRevD.71.032001.

[22] CDF Collaboration. The CDF Cherenkov luminosity monitor. Nuclear Inst. and Methods in Physics Research, A, 461(1-3):540-544, 2001. doi:10.1016/ S0168-9002(00)01294-8.

[23] CDF Collaboration. The performance of the CDF luminosity monitor. Nuclear Inst. and Methods in Physics Research, A, 494(1-3):57-62, 2002. doi:1016/S0168-9002(02) 01445-6. 
[24] CDF Collaboration. Intermediate silicon layers detector for the CDF experiment. Nuclear Inst. and Methods in Physics Research, A, 453(1-2):84-88, 2000. doi: 10.1016/S0168-9002(00)00610-0.

[25] CDF Collaboration. CDF Run II silicon tracking projects. Nuclear Inst. and Methods in Physics Research, A, 447(1-2):1-8, 2000. doi:10.1016/S0168-9002(00)00166-2.

[26] CDF Collaboration. CDF central outer tracker. Nuclear Inst. and Methods in Physics Research, A, 526(3):249-299, 2004. doi:10.1016/j.nima.2004.02.020.

[27] Balka, L. et al. The CDF central electromagnetic calorimeter. Nuclear Inst. and Methods in Physics Research, A, 267(2-3):272-279, 1988. doi:10.1016/0168-9002(88) 90474-3.

[28] CDF Collaboration. The CDF central and endwall hadron calorimeter. Nuclear Inst. and Methods in Physics Research, A, 267(2-3):301-314, 1988. doi:10.1016/ 0168-9002(88)90476-7.

[29] CDF Collaboration. The CDF calorimeter upgrade for Run IIb. Nuclear Inst. and Methods in Physics Research, A, 518(1-2):39-41, 2004. doi:10.1016/j.nima.2003.10. 018.

[30] CDF Collaboration. Online track processor for the CDF upgrade. IEEE Transactions on Nuclear Science, 49(3 Part 2):1063-1070, 2002. doi:10.1109/TNS.2002.1039615.

[31] Efron, J. Search for the Higgs Boson in the ZH $\rightarrow$ llbb Channel at CDF Run II. CDF Note, 8976, 2007. URL http://www-cdf.fnal.gov/thesis/cdf8976_ZHIlbb_ JZE_Thesis\%_2.ps.gz.

[32] CDF Collaboration. Determination of the jet energy scale at the Collider Detector at Fermilab. Nuclear Inst. and Methods in Physics Research, A, 566(2):375-412, 2006. doi:10.1016/j.nima.2006.05.269.

[33] CDF Collaboration. Search for the Higgs Boson Produced in Association with $Z \rightarrow$ $l^{+} l^{-}$in $p \bar{p}$ Collisions at $\sqrt{s}=1.96 \mathrm{TeV}$. Physical Review Letters, 101(25):251803 (pages 7), 2008. doi:10.1103/PhysRevLett.101.251803.

[34] CDF Collaboration. First Measurements of Inclusive $W$ and $Z$ Cross Sections from Run II of the Fermilab Tevatron Collider. Physical Review Letters, 94(9):091803, 2005. doi:10.1103/PhysRevLett.94.091803. 
[35] CDF Collaboration. Measurement of the $t \bar{t}$ Production Cross Section in $p \bar{p}$ Collisions at $\sqrt{s}=1.96 \mathrm{TeV}$ Using Dilepton Events. Physical Review Letters, 93(14):142001, 2004. doi:10.1103/PhysRevLett.93.142001.

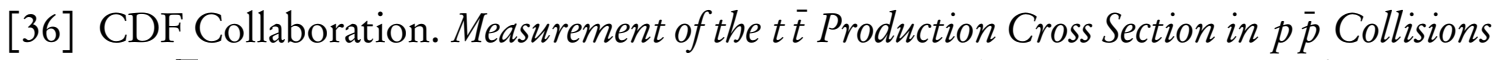
at $\sqrt{s}=1.96 \mathrm{TeV}$ using Lepton + Jets Events with Secondary Vertex $b$-tagging. Physical Review D, 71(5):052003, 2005. doi:10.1103/PhysRevD.71.052003.

[37] Mangano, M.L. et al. ALPGEN, a Generator for Hard Multiparton Processes in Hadronic Collisions. Journal of High Energy Physics, 07:001, 2003.

[38] Stump, D. et al. Inclusive jet production, parton distributions, and the search for new physics. Journal of High Energy Physics, 10:046, 2003. URL http://arxiv . org/ abs/hep-ph/0303013.

[39] Jayatilaka, B. A Measurement of the Top Quark Mass in the Dilepton Decay Channel at CDF II. CDF Note, 8481, 2006. URL http://www-cdf.fnal.gov/thesis/ cdf8481_me_dil_thesis.pdf.

[40] Lepage, G. A new algorithm for adaptive multidimensional integration. Journal of Computational Physics, 27(2):192-203, 1978.

[41] Campbell, J., Ellis, R.K., Maltoni, F., and Willenbrock, S. Production of a Z boson and two jets with one heavy-quark tag. Physical Review D, 73(5):054007, 2006. doi:10.1103/PhysRevD.73.054007.

[42] Campbell, J. and Ellis, R.K. Radiative corrections to Z $b \bar{b}$ production. Physical Review D, 62(11):114012, 2000. doi:10.1103/PhysRevD.62.114012.

[43] Alwall, J. et al. MadGraph/MadEvent v4: The New Web Generation. Journal of High Energy Physics, 09:028, 2007. 0706.2334, URL http://arxiv.org/abs/ 0706.2334.

[44] Mahlon, G. and Parke, S. Maximizing spin correlations in top quark pair production at the Tevatron. Physics Letters B, 411(1-2):173-179, 1997.

[45] Mahlon, G. and Parke, S. Improved spin basis for angular correlation studies in single top quark production at the Tevatron. Physical Review D, 55(11):7249, 1997.

[46] Wikipedia. Maximum likelihood, 2009. [Online; accessed 16-May2009], URL http://en.wikipedia.org/w/index.php?title=Maximum_ likelihood\&oldid=288802199. 
[47] Wikipedia. Bootstrapping (statistics), 2009. [Online; accessed 12-May2009], URL http://en.wikipedia.org/w/index.php?title=Bootstrapping_ (statistics) \&oldid=287033127.

[48] Efron, B. and Tibshirani, R.J. An Introduction to the Bootstrap. Chapman and Hall, New York, 1993.

[49] Barlow, R. Application of the Bootstrap Resampling Technique to Particle Physics Experiments. Technical report, MAN/HEP/99, 2000.

[50] Nelder, J. and Mead, R. A Simplex Method for Function Minimization. The Computer Journal, 7(4):308, 1965. doi:10.1093/comjnl/7.4.308.

[51] Wikipedia. Nelder-Mead method, 2009. [Online; accessed 29-April2009], URL http://en.wikipedia.org/w/index.php?title=Nelder-Mead_ method\&oldid=286921633.

[52] Feldman, G. and Cousins, R. Unified Approach to the Classical Statistical Analysis of Small Signals. Physical Review D, 57(7):3873-3889, 1998. doi:10.1103/PhysRevD. 57.3873 .

[53] Tevatron New Phenomena Higgs Working Group. Combined CDF and DZero Upper Limits on Standard Model Higgs-Boson Production with up to $4.2 \mathrm{fb}^{-1}$ of Data. 2009. URL http://arxiv. org/abs/0903.4001. 